\title{
Search for neutral Higgs bosons in $\mathrm{e}^{+} \mathrm{e}^{-}$collisions at $\sqrt{s}=183 \mathrm{GeV}$
}

\author{
DELPHI Collaboration
}

\begin{abstract}
Neutral Higgs bosons were searched for in the data collected by DELPHI at a centre-of-mass energy of $183 \mathrm{GeV}$, corresponding to an integrated luminosity of $54 \mathrm{pb}^{-1}$. The production of the lightest neutral Higgs boson with either an on-shell $\mathrm{Z}$ boson or a neutral pseudo-scalar Higgs boson was analysed. Lower limits at the $95 \%$ confidence level were obtained on the Higgs boson masses. The limits are $85.7 \mathrm{GeV} / c^{2}$ for the Standard Model Higgs boson and $74.4 \mathrm{GeV} / c^{2}$ for the scalar and $75.3 \mathrm{GeV} / c^{2}$ for the pseudo-scalar Higgs bosons as predicted by the minimal super-symmetric extension of the Standard Model with commonly used assumptions on the model parameters. These results significantly improve the limits reached with previous data.
\end{abstract}


P.Abreu ${ }^{21}, \quad$ W.Adam ${ }^{50}, \quad$ T.Adye ${ }^{36}, \quad$ P.Adzic ${ }^{11}, \quad$ I.Ajinenko ${ }^{42}, \quad$ Z.Albrecht ${ }^{17}, \quad$ T.Alderweireld ${ }^{2}, \quad$ G.D.Alekseev ${ }^{16}$, R.Alemany ${ }^{49}$, T.Allmendinger ${ }^{17}$, P.P.Allport ${ }^{22}$, S.Almehed ${ }^{24}$, U.Amaldi ${ }^{9}$, S.Amato ${ }^{47}$, E.G.Anassontzis ${ }^{3}$, P.Andersson ${ }^{44}$, A.Andreazza ${ }^{9}$, S.Andringa ${ }^{21}$, P.Antilogus ${ }^{25}$, W-D.Apel ${ }^{17}$, Y.Arnoud ${ }^{9}$, B.Åsman ${ }^{44}$, J-E.Augustinn ${ }^{25}$, A.Augustinus ${ }^{9}$,

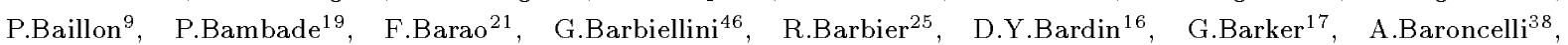
M.Battaglia ${ }^{15}$, M.Baubillier ${ }^{23}$, K-H.Becks ${ }^{52}$, M.Begalli ${ }^{6}$, P.Beilliere ${ }^{8}$, Yu.Belokopytov ${ }^{9,53}$, A.C.Benvenuti ${ }^{5}$, C.Berat ${ }^{14}$, M.Berggren $^{25}$, D.Bertini2 ${ }^{25}$, D.Bertrand ${ }^{2}$, M.Besancon ${ }^{39}$, F.Bianchi ${ }^{45}$, M.Bigi ${ }^{45}$, M.S.Bilenky ${ }^{16}$, M-A.Bizouard ${ }^{19}$, D.Bloch $^{10}$, H.M.Blom ${ }^{30}$, M.Bonesini2 ${ }^{2}$, W.Bonivento ${ }^{27}$, M.Boonekamp ${ }^{39}$, P.S.L.Booth ${ }^{22}$, A.W.Borgland ${ }^{4}$, G.Borisov ${ }^{19}$, C.Bosio ${ }^{41}$, O.Botner ${ }^{48}$, E.Boudinov ${ }^{30}$, B.Bouquet ${ }^{19}$, C.Bourdarios ${ }^{19}$, T.J.V.Bowcock ${ }^{22}$, I.Boyko ${ }^{16}$, I.Bozovic ${ }^{11}$, M.Bozzo ${ }^{13}$, P.Branchini ${ }^{38}$, T.Brenke ${ }^{52}$, R.A.Brenner ${ }^{48}, \quad$ P.Bruckman ${ }^{18}, \quad$ J-M.Brunet ${ }^{8}, \quad$ L.Bugge $^{32}, \quad$ T.Buran ${ }^{32}$, T.Burgsmueller ${ }^{52}$, P.Buschmann ${ }^{52}$, S.Cabrera ${ }^{49}$, M.Caccia $^{27}$, M.Calvi ${ }^{27}$, T.Camporesi ${ }^{9}$, V.Canale ${ }^{37}$, F.Carena ${ }^{9}$, L.Carroll $^{22}$, C.Caso ${ }^{13}$, M.V.Castillo Gimenez ${ }^{49}$, A.Cattai ${ }^{9}$, F.R.Cavallo ${ }^{5}$, V.Chabaud ${ }^{9}$, Ph.Charpentier ${ }^{9}$, L.Chaussard ${ }^{25}$, P.Checchia $^{35}$, G.A.Chelkov ${ }^{16}$, R.Chierici ${ }^{45}$, P.Chliapnikov ${ }^{42}$, P.Chochula ${ }^{7}$, V.Chorowicz ${ }^{25}$, J.Chudoba ${ }^{29}$, K.Cieslik ${ }^{18}$, P.Collins $^{9}$, R.Contri ${ }^{13}$, E.Cortina ${ }^{49}$, G.Cosme ${ }^{19}$, F.Cossutti ${ }^{9}$, J-H.Cowell ${ }^{22}$, H.B.Crawley ${ }^{1}$, D.Crennell ${ }^{36}$, S.Crepe ${ }^{14}$, G.Crosetti $^{13}$, J.Cuevas Maestro ${ }^{33}$, S.Czellar ${ }^{15}$, M.Davenport ${ }^{9}$, W.Da Silva ${ }^{23}$, A.Deghorain ${ }^{2}$, G.Della Ricca ${ }^{46}$, P.Delpierre ${ }^{26}$, N.Demaria ${ }^{9}$, A.De Angelis ${ }^{9}$, W.De Boer ${ }^{17}$, S.De Brabandere ${ }^{2}$, C.De Clercq ${ }^{2}$, B.De Lotto ${ }^{46}$, A.De Min ${ }^{35}$, L.De Paula ${ }^{47}$, H.Dijkstra ${ }^{9}$, L.Di Ciaccio ${ }^{37,9}$, J.Dolbeau ${ }^{8}$, K.Doroba ${ }^{51}$, M.Dracos ${ }^{10}$, J.Drees ${ }^{52}$, M.Dris ${ }^{31}$, A.Duperrin ${ }^{25}$, J-D.Durand ${ }^{9}$, G.Eigen ${ }^{4}$, T.Ekelof ${ }^{48}$, G.Ekspong ${ }^{44}$, M.Ellert ${ }^{48}$, M.Elsing ${ }^{9}$, J-P.Engel ${ }^{10}$, B.Erzen ${ }^{43}$, M.Espirito Santo ${ }^{21}$, E.Falk $^{24}$, G.Fanourakis ${ }^{11}$, D.Fassouliotis ${ }^{11}$, J.Fayot ${ }^{23}$, M.Feindt ${ }^{17}$, P.Ferrari ${ }^{27}$, A.Ferrer ${ }^{49}$, E.Ferrer-Ribas ${ }^{19}$, S.Fichet ${ }^{23}$, A.Firestone ${ }^{1}$, U.Flagmeyer ${ }^{52}$, H.Foeth ${ }^{9}$, E.Fokitis ${ }^{31}$, F.Fontanelli ${ }^{13}$, B.Franek ${ }^{36}$, A.G.Frodesen ${ }^{4}$, R.Fruhwirth ${ }^{50}$, F.Fulda-Quenzer ${ }^{19}$, J.Fuster ${ }^{49}$, A.Galloni ${ }^{22}$, D.Gamba ${ }^{45}, \quad$ S.Gamblin ${ }^{19}$, M.Gandelman ${ }^{47}, \quad$ C.Garcia ${ }^{49}, \quad$ C.Gaspar ${ }^{9}$, M.Gaspar ${ }^{47}$, U.Gasparini ${ }^{35}$, Ph.Gavillet ${ }^{9}$, E.N.Gazis ${ }^{31}$, D.Gele ${ }^{10}$, L.Gerdyukov ${ }^{42}$, N.Ghodbane ${ }^{25}$, I.Gil ${ }^{49}$, F.Glege ${ }^{52}$, R.Gokieli ${ }^{9,51}$, B.Golob ${ }^{43}$, G.Gomez-Ceballos ${ }^{40}$, P.Goncalves ${ }^{21}$, I.Gonzalez Caballero ${ }^{40}$, G.Gopal ${ }^{36}, \quad$ L.Gorn ${ }^{1,54}$, M.Gorski ${ }^{51}$, Yu.Gouz ${ }^{42}$, V.Gracco ${ }^{13}$, J.Grahl ${ }^{1}$, E.Graziani ${ }^{38}$, C.Green ${ }^{22}$, H-J.Grimm ${ }^{17}$, P.Gris ${ }^{39}$, G.Grosdidier ${ }^{19}$, K.Grzelak ${ }^{51}$, M.Gunther ${ }^{48}$, J.Guy ${ }^{36}$, F.Hahn ${ }^{9}$, S.Hahn ${ }^{52}$, S.Haider ${ }^{9}$, A.Hallgren ${ }^{48}$, K.Hamacher ${ }^{52}$, J.Hansen ${ }^{32}$, F.J.Harris ${ }^{34}$, V.Hedberg ${ }^{24}$, S.Heising ${ }^{17}$, J.J.Hernandez ${ }^{49}$, P.Herquet ${ }^{2}$, H.Herr ${ }^{9}$, T.L.Hessing ${ }^{34}$, J.-M.Heuser ${ }^{52}$, E.Higon ${ }^{49}$, S-O.Holmgren ${ }^{44}$, P.J.Holt ${ }^{34}$, S.Hoorelbeke ${ }^{2}$, M.Houlden ${ }^{22}$, J.Hrubec ${ }^{50}$, K.Huet ${ }^{2}$, G.J.Hughes ${ }^{22}$, K.Hultqvist ${ }^{44}$, J.N.Jackson ${ }^{22}$, R.Jacobsson ${ }^{9}$, P.Jalocha ${ }^{9}$, R.Janik ${ }^{7}$, Ch.Jarlskog ${ }^{24}$, G.Jarlskog ${ }^{24}$, P.Jarry ${ }^{39}$, B.Jean-Marie ${ }^{19}$, E.K.Johansson ${ }^{44}$, P.Jonsson ${ }^{25}$, C.Joram ${ }^{9}$, P.Juillot ${ }^{10}$, F.Kapusta ${ }^{23}$, K.Karafasoulis ${ }^{11}$, S.Katsanevas ${ }^{25}$, E.C.Katsoufis ${ }^{31}$, R.Keranen ${ }^{17}$, B.P.Kersevan ${ }^{43}$, B.A.Khomenko ${ }^{16}$, N.N.Khovanski ${ }^{16}$, A.Kiiskinen ${ }^{15}$, B.King ${ }^{22}$, A.Kinvig ${ }^{22}$, N.J.Kjaer ${ }^{30}$, O.Klapp $^{52}$, H.Klein ${ }^{9}$, P.Kluit ${ }^{30}$, P.Kokkinias ${ }^{11}$, M.Koratzinos ${ }^{9}$, V.Kostioukhine ${ }^{42}$, C.Kourkoumelis ${ }^{3}$, O.Kouznetsov ${ }^{39}$, E.Kriznic $^{43}$, J.Krstic ${ }^{11}$, Z.Krumstein ${ }^{16}$, P.Kubinec $^{7}$, J.Kurowska ${ }^{51}$, K.Kurvinen ${ }^{15}$, J.W.Lamsa ${ }^{1}, \quad$ D.W.Lane ${ }^{1}$, P.Langefeld ${ }^{52}$, V.Lapin ${ }^{42}$, J-P.Laugier ${ }^{39}$, R.Lauhakangas ${ }^{15}$, G.Leder ${ }^{50}$, F.Ledroit ${ }^{14}$, V.Lefebure ${ }^{2}$, L.Leinonen ${ }^{44}$, A.Leisos $^{11}$, R.Leitner ${ }^{29}$, G.Lenzen ${ }^{52}$, V.Lepeltier ${ }^{19}$, T.Lesiak ${ }^{18}$, M.Lethuillier ${ }^{39}$, J.Libby ${ }^{34}$, D.Liko ${ }^{9}$, A.Lipniacka ${ }^{44}$, I.Lippi $^{35}, \quad$ B.Loerstad ${ }^{24}$, J.G.Loken ${ }^{34}$, J.H.Lopes ${ }^{47}, \quad$ J.M.Lopez ${ }^{40}, \quad$ R.Lopez-Fernandez ${ }^{14}, \quad$ D.Loukas ${ }^{11}, \quad$ P.Lutz ${ }^{39}$, L.Lyons $^{34}$, J.MacNaughton ${ }^{50}$, J.R.Mahon ${ }^{6}$, A.Maio ${ }^{21}$, A.Malek ${ }^{52}$, T.G.M.Malmgren ${ }^{44}$, V.Malychev ${ }^{16}$, F.Mandl ${ }^{50}$, J.Marco $^{40}$, R.Marco ${ }^{40}$, B.Marechal ${ }^{47}$, M.Margoni ${ }^{35}$, J-C.Marin ${ }^{9}$, C.Mariotti ${ }^{9}$, A.Markou ${ }^{11}$, C.Martinez-Rivero ${ }^{19}$, F.Martinez-Vidal ${ }^{49}$, S.Marti i Garcia ${ }^{9}$, J.Masik ${ }^{12}$, N.Mastroyiannopoulos ${ }^{11}$, F.Matorras ${ }^{40}$, C.Matteuzzi ${ }^{27}$, G.Matthiae ${ }^{37}$, F.Mazzucato ${ }^{35}$, M.Mazzucato ${ }^{35}$, M.Mc Cubbin ${ }^{22}$, R.Mc Kay ${ }^{1}$, R.Mc Nulty ${ }^{22}$ ， G.Mc Pherson ${ }^{22}$, C.Meroni ${ }^{27}$, W.T.Meyer ${ }^{1}$, A.Miagkov ${ }^{42}$, E.Migliore ${ }^{45}$, L.Mirabito ${ }^{25}$, W.A.Mitaroff ${ }^{50}$, U.Mjoernmark ${ }^{24}$, T.Moa ${ }^{44}$, M.Moch ${ }^{17}$, R.Moeller ${ }^{28}$, K.Moenig ${ }^{9}$, M.R.Monge ${ }^{13}$, X.Moreau ${ }^{23}$, P.Morettini ${ }^{13}$, G.Morton ${ }^{34}$, U.Mueller ${ }^{52}$, K.Muenich ${ }^{52}$, M.Mulders ${ }^{30}$, C.Mulet-Marquis ${ }^{14}$, R.Muresan ${ }^{24}$, W.J.Murray ${ }^{36}$, B.Muryn ${ }^{14,18}$, G.Myatt $^{34}$, T.Myklebust ${ }^{32}$, F.Naraghi ${ }^{14}$, F.L.Navarria ${ }^{5}$, S.Navas ${ }^{49}$, K.Nawrocki ${ }^{51}$, P.Negri ${ }^{27}$, N.Neufeld ${ }^{9}$, N.Neumeister ${ }^{50}$, R.Nicolaidou ${ }^{39}$, B.S.Nielsen ${ }^{28}$, M.Nikolenko ${ }^{10,16}$, V.Nomokonov ${ }^{15}$, A.Normand ${ }^{22}$, A.Nygren ${ }^{24}$, V.Obraztsov ${ }^{42}$, A.G.Olshevski ${ }^{16}$, A.Onofre ${ }^{21}$, R.Orava $^{15}$, G.Orazi $^{10}$, K.Osterberg ${ }^{15}$, A.Ouraou ${ }^{39}$, M.Paganoni ${ }^{27}$, S.Paiano ${ }^{5}$, R.Pain ${ }^{23}$, R.Paiva ${ }^{21}$, J.Palacios ${ }^{34}$, H.Palka ${ }^{18}$, Th.D.Papadopoulou ${ }^{31}$, K.Papageorgiou ${ }^{11}$, L.Pape ${ }^{9}$, C.Parkes ${ }^{9}$, F.Parodi ${ }^{13}$, U.Parzefall ${ }^{22}$, A.Passeri ${ }^{38}$, O.Passon ${ }^{52}$, M.Pegoraro $^{35}$, L.Peralta ${ }^{21}$, M.Pernicka ${ }^{50}$, A.Perrotta ${ }^{5}$, C.Petridou ${ }^{46}$, A.Petrolini ${ }^{13}$, H.T.Phillips ${ }^{36}, \quad$ F.Pierre ${ }^{39}$, M.Pimenta ${ }^{21}$, E.Piotto ${ }^{27}$, T.Podobnik ${ }^{43}$, M.E.Pol ${ }^{6}, \quad$ G.Polok ${ }^{18}$, P.Poropat ${ }^{46}$, V.Pozdniakov ${ }^{16}$, P.Privitera ${ }^{37}$,

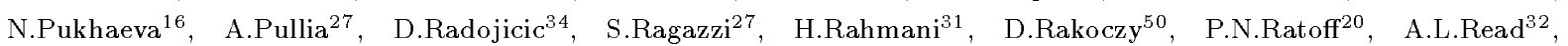
P.Rebecchi $^{9}$, N.G.Redaelli ${ }^{27}$, M.Regler ${ }^{50}$, D.Reid ${ }^{30}, \quad$ R.Reinhardt ${ }^{52}$, P.B.Renton ${ }^{34}$, L.K.Resvanis ${ }^{3}, \quad$ F.Richard ${ }^{19}$, J.Ridky $^{12}$, G.Rinaudo ${ }^{45}$, O.Rohne ${ }^{32}$, A.Romero ${ }^{45}$, P.Ronchese ${ }^{35}$, E.I.Rosenberg ${ }^{1}$, P.Rosinsky ${ }^{7}$, P.Roudeau $^{19}$, T.Rovelli ${ }^{5}$, Ch.Royon $^{39}$, V.Ruhlmann-Kleider ${ }^{39}$, A.Ruiz ${ }^{40}$, H.Saarikko ${ }^{15}$, Y.Sacquin ${ }^{39}$, A.Sadovsky ${ }^{16}$, G.Sajot ${ }^{14}$, J.Salt ${ }^{49}$, D.Sampsonidis ${ }^{11}$, M.Sannino ${ }^{13}$, H.Schneider ${ }^{17}$, Ph.Schwemling ${ }^{23}$, U.Schwickerath ${ }^{17}$, M.A.E.Schyns ${ }^{52}, \quad$ F.Scuri ${ }^{46}$,

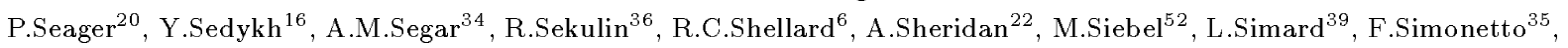
A.N.Sisakian ${ }^{16}$, G.Smadja ${ }^{25}$, O.Smirnova ${ }^{24}$, G.R.Smith ${ }^{36}$, A.Sokolov ${ }^{42}$, A.Sopczak ${ }^{17}$, R.Sosnowski ${ }^{51}$, T.Spassov ${ }^{21}$,

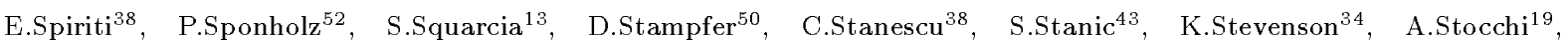
J.Strauss $^{50}$, R.Strub ${ }^{10}$, B.Stugu ${ }^{4}$, M.Szczekowski ${ }^{51}$, M.Szeptycka ${ }^{51}$, T.Tabarelli2 ${ }^{27}$, O.Tchikilev ${ }^{42}$, F.Tegenfeldt ${ }^{48}$, F.Terranova ${ }^{27}$, J.Thomas ${ }^{34}$, J.Timmermans ${ }^{30}$, N.Tinti ${ }^{5}$, L.G.Tkatchev ${ }^{16}$, S.Todorova ${ }^{10}$, A.Tomaradze ${ }^{2}$, B.Tome ${ }^{21}$,

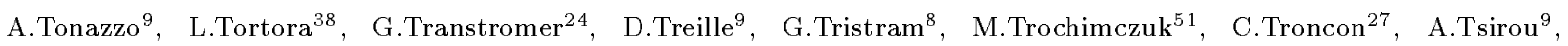


M-L.Turluer ${ }^{39}$, I.A.Tyapkin ${ }^{16}$, S.Tzamarias ${ }^{11}$, B.Ueberschaer ${ }^{52}$, O.Ullaland ${ }^{9}$, V.Uvarov ${ }^{42}$, G.Valenti ${ }^{5}$, E.Vallazza ${ }^{46}$, C.Vander Velde ${ }^{2}, \quad$ G.W.Van Apeldoorn ${ }^{30}, \quad$ P.Van Dam $^{30}, \quad$ J.Van Eldik ${ }^{30}, \quad$ A.Van Lysebetten ${ }^{2}, \quad$ I.Van Vulpen ${ }^{30}$, N.Vassilopoulos ${ }^{34}$, G.Vegni ${ }^{27}$, L.Ventura ${ }^{35}$, W.Venus ${ }^{36,9}$, F.Verbeure ${ }^{2}$, M.Verlato ${ }^{35}$, L.S.Vertogradov ${ }^{16}$, V.Verzi $^{37}$,

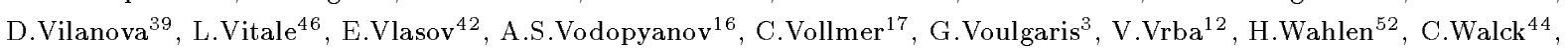
C.Weiser $^{17}$, D.Wicke ${ }^{52}$, J.H.Wickens ${ }^{2}$, G.R.Wilkinson ${ }^{9}$, M.Winter ${ }^{10}$, M.Witek ${ }^{18}$, G.Wolf ${ }^{9}$, J.Yi ${ }^{1}$, O.Yushchenko ${ }^{42}$, A.Zalewska ${ }^{18}$, P.Zalewski ${ }^{51}$, D.Zavrtanik ${ }^{43}$, E.Zevgolatakos ${ }^{11}$, N.I.Zimin ${ }^{16,24}$, G.C.Zucchelli ${ }^{44}$, G.Zumerle ${ }^{35}$

\footnotetext{
${ }^{1}$ Department of Physics and Astronomy, Iowa State University, Ames IA 50011-3160, USA

${ }^{2}$ Physics Department, Univ. Instelling Antwerpen, Universiteitsplein 1, BE-2610 Wilrijk, Belgium and IIHE, ULB-VUB, Pleinlaan 2, BE-1050 Brussels, Belgium

and Faculté des Sciences, Univ. de l'Etat Mons, Av. Maistriau 19, BE-7000 Mons, Belgium

${ }^{3}$ Physics Laboratory, University of Athens, Solonos Str. 104, GR-10680 Athens, Greece

${ }^{4}$ Department of Physics, University of Bergen, Allégaten 55, NO-5007 Bergen, Norway

${ }^{5}$ Dipartimento di Fisica, Università di Bologna and INFN, Via Irnerio 46, IT-40126 Bologna, Italy

${ }^{6}$ Centro Brasileiro de Pesquisas Físicas, rua Xavier Sigaud 150, BR-22290 Rio de Janeiro, Brazil and Depto. de Física, Pont. Univ. Católica, C.P. 38071 BR-22453 Rio de Janeiro, Brazil

and Inst. de Física, Univ. Estadual do Rio de Janeiro, rua São Francisco Xavier 524, Rio de Janeiro, Brazil

${ }^{7}$ Comenius University, Faculty of Mathematics and Physics, Mlynska Dolina, SK-84215 Bratislava, Slovakia

${ }^{8}$ Collège de France, Lab. de Physique Corpusculaire, IN2P3-CNRS, FR-75231 Paris Cedex 05, France

${ }^{9} \mathrm{CERN}, \mathrm{CH}-1211$ Geneva 23, Switzerland

${ }^{10}$ Institut de Recherches Subatomiques, IN2P3 - CNRS/ULP - BP20, FR-67037 Strasbourg Cedex, France

${ }^{11}$ Institute of Nuclear Physics, N.C.S.R. Demokritos, P.O. Box 60228, GR-15310 Athens, Greece

${ }^{12}$ FZU, Inst. of Phys. of the C.A.S. High Energy Physics Division, Na Slovance 2, CZ-180 40, Praha 8, Czech Republic

${ }^{13}$ Dipartimento di Fisica, Università di Genova and INFN, Via Dodecaneso 33, IT-16146 Genova, Italy

${ }^{14}$ Institut des Sciences Nucléaires, IN2P3-CNRS, Université de Grenoble 1, FR-38026 Grenoble Cedex, France

${ }^{15}$ Helsinki Institute of Physics, HIP, P.O. Box 9, FI-00014 Helsinki, Finland

${ }^{16}$ Joint Institute for Nuclear Research, Dubna, Head Post Office, P.O. Box 79, RU-101 000 Moscow, Russian Federation

${ }^{17}$ Institut für Experimentelle Kernphysik, Universität Karlsruhe, Postfach 6980, DE-76128 Karlsruhe, Germany

${ }^{18}$ Institute of Nuclear Physics and University of Mining and Metalurgy, Ul. Kawiory 26a, PL-30055 Krakow, Poland

${ }^{19}$ Université de Paris-Sud, Lab. de l'Accélérateur Linéaire, IN2P3-CNRS, Bât. 200, FR-91405 Orsay Cedex, France

${ }^{20}$ School of Physics and Chemistry, University of Lancaster, Lancaster LA1 4YB, UK

${ }^{21}$ LIP, IST, FCUL - Av. Elias Garcia, 14-1 ${ }^{\circ}$, PT-1000 Lisboa Codex, Portugal

${ }^{22}$ Department of Physics, University of Liverpool, P.O. Box 147, Liverpool L69 3BX, UK

${ }^{23}$ LPNHE, IN2P3-CNRS, Univ. Paris VI et VII, Tour 33 (RdC), 4 place Jussieu, FR-75252 Paris Cedex 05, France

${ }^{24}$ Department of Physics, University of Lund, Sölvegatan 14, SE-223 63 Lund, Sweden

${ }^{25}$ Université Claude Bernard de Lyon, IPNL, IN2P3-CNRS, FR-69622 Villeurbanne Cedex, France

${ }^{26}$ Univ. d'Aix - Marseille II - CPP, IN2P3-CNRS, FR-13288 Marseille Cedex 09, France

${ }^{27}$ Dipartimento di Fisica, Università di Milano and INFN, Via Celoria 16, IT-20133 Milan, Italy

${ }^{28}$ Niels Bohr Institute, Blegdamsvej 17, DK-2100 Copenhagen $\varnothing$, Denmark

${ }^{29} \mathrm{NC}$, Nuclear Centre of MFF, Charles University, Areal MFF, V Holesovickach 2, CZ-180 00, Praha 8, Czech Republic

${ }^{30}$ NIKHEF, Postbus 41882, NL-1009 DB Amsterdam, The Netherlands

${ }^{31}$ National Technical University, Physics Department, Zografou Campus, GR-15773 Athens, Greece

32 Physics Department, University of Oslo, Blindern, NO-1000 Oslo 3, Norway

${ }^{33}$ Dpto. Fisica, Univ. Oviedo, Avda. Calvo Sotelo s/n, ES-33007 Oviedo, Spain

${ }^{34}$ Department of Physics, University of Oxford, Keble Road, Oxford OX1 3RH, UK

${ }^{35}$ Dipartimento di Fisica, Università di Padova and INFN, Via Marzolo 8, IT-35131 Padua, Italy

${ }^{36}$ Rutherford Appleton Laboratory, Chilton, Didcot OX11 OQX, UK

${ }^{37}$ Dipartimento di Fisica, Università di Roma II and INFN, Tor Vergata, IT-00173 Rome, Italy

${ }^{38}$ Dipartimento di Fisica, Università di Roma III and INFN, Via della Vasca Navale 84, IT-00146 Rome, Italy

${ }^{39}$ DAPNIA/Service de Physique des Particules, CEA-Saclay, FR-91191 Gif-sur-Yvette Cedex, France

${ }^{40}$ Instituto de Fisica de Cantabria (CSIC-UC), Avda. los Castros s/n, ES-39006 Santander, Spain

${ }^{41}$ Dipartimento di Fisica, Università degli Studi di Roma La Sapienza, Piazzale Aldo Moro 2, IT-00185 Rome, Italy

${ }^{42}$ Inst. for High Energy Physics, Serpukov P.O. Box 35, Protvino, (Moscow Region), Russian Federation

${ }^{43}$ J. Stefan Institute, Jamova 39, SI-1000 Ljubljana, Slovenia and Laboratory for Astroparticle Physics, Nova Gorica Polytechnic, Kostanjeviska 16a, SI-5000 Nova Gorica, Slovenia, and Department of Physics, University of Ljubljana, SI-1000 Ljubljana, Slovenia

${ }^{44}$ Fysikum, Stockholm University, Box 6730, SE-113 85 Stockholm, Sweden

${ }^{45}$ Dipartimento di Fisica Sperimentale, Università di Torino and INFN, Via P. Giuria 1, IT-10125 Turin, Italy

${ }^{46}$ Dipartimento di Fisica, Università di Trieste and INFN, Via A. Valerio 2, IT-34127 Trieste, Italy and Istituto di Fisica, Università di Udine, IT-33100 Udine, Italy

${ }^{47}$ Univ. Federal do Rio de Janeiro, C.P. 68528 Cidade Univ., Ilha do Fundão BR-21945-970 Rio de Janeiro, Brazil

${ }^{48}$ Department of Radiation Sciences, University of Uppsala, P.O. Box 535, SE-751 21 Uppsala, Sweden

${ }^{49}$ IFIC, Valencia-CSIC, and D.F.A.M.N., U. de Valencia, Avda. Dr. Moliner 50, ES-46100 Burjassot (Valencia), Spain

${ }^{50}$ Institut für Hochenergiephysik, Österr. Akad. d. Wissensch., Nikolsdorfergasse 18, AT-1050 Vienna, Austria

${ }^{51}$ Inst. Nuclear Studies and University of Warsaw, Ul. Hoza 69, PL-00681 Warsaw, Poland

${ }^{52}$ Fachbereich Physik, University of Wuppertal, Postfach 100 127, DE-42097 Wuppertal, Germany

${ }^{53}$ On leave of absence from IHEP Serpukhov

${ }^{54}$ Now at University of Florida
} 


\section{Introduction}

In the framework of the Standard Model (SM) there is one physical Higgs boson, $\mathrm{H}$, which is a neutral CP-even scalar. At LEP II the most likely production process is through the s-channel, $\mathrm{e}^{+} \mathrm{e}^{-} \rightarrow \mathrm{Z}^{*} \rightarrow \mathrm{ZH}$. There are also $\mathrm{W}^{+} \mathrm{W}^{-}$and $\mathrm{ZZ}$ fusion t-channel production processes in some of the channels described here, but their contribution to the cross-section is at most $10 \%$.

In the Minimal Super-symmetric Standard Model (MSSM) five physical Higgs bosons are predicted, but only two are likely to be accessible at LEP II. There is one scalar boson, the $\mathrm{h}$, analogous to the SM Higgs, but bounded in mass to be less than $m_{\mathrm{Z}}$ at tree level. After radiative corrections its mass may be larger. The results of the search for the SM Higgs are also interpreted in terms of this boson. The CP-odd pseudo-scalar, $\mathrm{A}$, would be produced mostly in an hA state.

We have previously [1] excluded a Standard Model H with mass less than $66.2 \mathrm{GeV} / c^{2}$, and set limits on $\mathrm{h}$ and A of the MSSM of $59.5 \mathrm{GeV} / c^{2}$ and $51.0 \mathrm{GeV} / c^{2}$ respectively. The present analyses therefore concentrate on masses between these and the kinematic limit. Note that the LEP Higgs working group [2] has found a mass limit on $\mathrm{H}$ of $77.5 \mathrm{GeV} / c^{2}$ when combining the limits of the four experiments from data taken up to $172 \mathrm{GeV}$.

All known decays of the $\mathrm{Z}$ boson have been taken into account (hadrons, charged leptons and neutrinos) while the analyses have been optimized either for decays of the Higgs into $b \bar{b}$, making use of the expected high branching fraction of this mode, or for Higgs boson decays into a pair of $\tau$ 's.

The analysis is divided according to the decay products of the Higgs and $\mathrm{Z}$ bosons. Some common features are discussed in Sect. 4, the $\mathrm{H}^{+} \mu^{-}$and $\mathrm{He}^{+} \mathrm{e}^{-}$channels in Sect. 5, $\mathrm{H} \nu \bar{\nu}$ in Sect. 6, and channels involving jets and $\tau$ 's in Sect. 7. Purely hadronic final states are discussed in Sect. 8. The results are presented in Sect. 9.

\section{Collected data overview}

For most of the data collected in 1997, LEP was running at energies around $183 \mathrm{GeV}$. DELPHI recorded an integrated luminosity of $54.0 \pm 0.5 \mathrm{pb}^{-1}$ at a mean energy of $182.7 \mathrm{GeV}$.

Large numbers of background and signal events have been produced by Monte Carlo simulation using the DELPHI detector simulation program [3]. The available statistics of the background are given in Table 1. The background events have been generated with PYTHIA [4] and KORALZ [5] for $\left(\mathrm{e}^{+} \mathrm{e}^{-} \rightarrow \mathrm{q} \overline{\mathrm{q}}(\gamma)\right)$, PYTHIA and EXCALIBUR [6] for the four-fermion background and TWOGAM [7] and BDK [8] for two-photon processes. BABAMC [9] is used to simulate Bhabha events in the main acceptance region. In general PYTHIA has been used for the four-fermion background rates unless ZZ and Zee survive at significant rates, in which case the interference between these states must be handled by a 4 fermion generator. Some studies have also been done with the GRC4F [10] and WPHACT [11] generators. It is always assumed that 'gluonic returns', where a quark loop from the s-channel produces two gluons and an on-shell $\mathrm{Z}$, are negligible.

Signal events have been produced using the HZHA [12] generator. For the SM process the Higgs mass has been varied in $5 \mathrm{GeV} / c^{2}$ steps from $60 \mathrm{GeV} / c^{2}$ to $95 \mathrm{GeV} / c^{2}$, while for hA of the MSSM, the A mass has been varied between 55 and $85 \mathrm{GeV} / c^{2}$ with $\tan \beta$ (the ratio of the vacuum expectation values of the two doublets) either 2 or 20 . This fixes the $h$ mass. 
Type of background generator cross section simulated luminosity

\begin{tabular}{cccc}
\hline $\mathrm{e}^{+} \mathrm{e}^{-} \rightarrow \mathrm{q} \overline{\mathrm{q}}(\gamma)$ & PYTHIA & $107.5 \mathrm{pb}$ & $10000 \mathrm{pb}^{-1}$ \\
\hline $\mathrm{e}^{+} \mathrm{e}^{-} \rightarrow \mathrm{W}^{+} \mathrm{W}^{-}$ & PYTHIA & $15.4 \mathrm{pb}$ & $2800 \mathrm{pb}^{-1}$ \\
$\mathrm{e}^{+} \mathrm{e}^{-} \rightarrow Z^{*}$ or Z $\gamma^{*}$ & PYTHIA & $1.25 \mathrm{pb}$ & $13500 \mathrm{pb}^{-1}$ \\
$\mathrm{e}^{+} \mathrm{e}^{-} \rightarrow$ Zee & PYTHIA & $6.8 \mathrm{pb}$ & $375 \mathrm{pb}^{-1}$ \\
$\mathrm{e}^{+} \mathrm{e}^{-} \rightarrow$ We $\nu$ & PYTHIA & $0.6 \mathrm{pb}$ & $15000 \mathrm{pb}^{-1}$ \\
\hline $\mathrm{e}^{+} \mathrm{e}^{-} \rightarrow 4$ fermion & EXCALIBUR & $18.46 \mathrm{pb}$ & $2400 \mathrm{pb}^{-1}$ \\
\hline Bhabha events & BABAMC & $1261 \mathrm{pb}$ & $72 \mathrm{pb}^{-1}$ \\
$\gamma \gamma_{\mathrm{QCD}}$ & TWOGAM & $2307 \mathrm{pb}$ & $154 \mathrm{pb}^{-1}$ \\
$\gamma \gamma_{\mathrm{QPM}}$ & BDK & $988 \mathrm{pb}$ & $729 \mathrm{pb}^{-1}$ \\
$\gamma \gamma_{\mathrm{VDM}}$ & TWOGAM & $7500 \mathrm{pb}$ & $56 \mathrm{pb}^{-1}$ \\
\hline
\end{tabular}

Table 1: Statistics for the various simulated backgrounds. The cross-sections refer to an energy of $183 \mathrm{GeV}$.

The ZH simulated samples are organised by the Higgs and Z boson decay modes. For $\mathrm{He}^{+} \mathrm{e}^{-}, \mathrm{H} \mu^{+} \mu^{-}$and $\mathrm{H} \nu \bar{\nu}$ the natural mix of $\mathrm{H}$ decay modes is permitted. In the $\mathrm{Hq} \overline{\mathrm{q}}$ channel the $\tau \tau$ decay mode is removed, and we generate separately the two channels involving $\tau$ leptons for which one of the bosons is forced to decay to $\tau$ 's and the other hadronically. Finally, for the hA simulations final states involving either four b quarks or two b quarks and two $\tau$ 's are simulated. Efficiencies are defined relative to these states.

\section{The DELPHI detector}

The detector consists of a cylindrical part covering the barrel region (with polar angle, $\theta$, typically from $40^{\circ}$ to $140^{\circ}$ ) and two end caps covering the forward regions. A large super-conducting solenoid provides a magnetic field of $1.2 \mathrm{~T}$ inside the central tracking volume. In the barrel region, tracks of charged particles are reconstructed in the microvertex detector, the inner detector, the time projection chamber (TPC) and the outer detector, reaching an average inverse momentum resolution, $\sigma(1 / p)$, of $0.57 \cdot 10^{-3}(\mathrm{GeV} / c)^{-1}$ for $45 \mathrm{GeV} / c$ muons. In the forward region, the reconstruction is achieved by the time projection chamber, the inner detector and forward drift chambers called A (which are just behind the TPC end-plates) and B (which are in front of the forward electromagnetic calorimeter) with a momentum resolution of $1.31 \cdot 10^{-3}(\mathrm{GeV} / c)^{-1}$. The polar angle acceptance of the whole tracking system is $20^{\circ}<\theta<160^{\circ}$.

The microvertex detector, as well as improving the momentum resolution by a factor of two, also provides precise measurements of impact parameters and secondary vertices. As compared to the setup described in [13], the three layers of the microvertex detector have been extended down to $25^{\circ}$ in $\theta$. Typical precisions of the impact parameter measurements are $26 \mu \mathrm{m}$ in the transverse plane, and $47 \mu \mathrm{m}$ along the beam direction for high momentum particles emitted at $90^{\circ}$ in $\theta$. The time projection chamber can also provide charged particle identification by measurement of the energy loss.

Neutral and charged particle energies are measured in the electromagnetic and hadronic calorimeters, whose coverage in $\theta$ starts at $8^{\circ}$ and $11^{\circ}$ respectively. The fractional 
energy resolutions are $0.32 / \sqrt{E}$ and $0.12 / \sqrt{E}$ in the barrel and forward electromagnetic calorimeters, respectively, and $1.3 / \sqrt{E}(E$ in $\mathrm{GeV})$ in the hadron calorimeter. Particles emitted at small angles are detected in the small angle calorimeter (STIC) devoted to luminosity measurement, whose acceptance lies between $1.69^{\circ}$ and $10.8^{\circ}$. The hermeticity of the electromagnetic calorimetry is improved by photon taggers which cover the gap between the barrel and forward regions at $\theta \simeq 40^{\circ}$, the weak region at $\theta \simeq 90^{\circ}$ and some azimuthal gaps in the barrel calorimeter acceptance.

Finally, muons are identified by their penetration through the iron yoke of the hadron calorimeter to planes of drift chambers located partly inside and partly outside the yoke. The barrel region is equipped with three sets of drift chambers while the end caps each contain two planes. One surrounding layer of limited streamer tubes completes the coverage between the barrel and forward regions at $\theta \simeq 50^{\circ}$.

More details about the apparatus and its performance can be found in references $[13,14]$.

The luminosity used by most analyses was $54 \mathrm{pb}^{-1}$. Following the quality control on the DELPHI subdetectors, the requirement of full detector performance reduced the luminosities in the $\mathrm{H} \nu \bar{\nu}$ and $\mathrm{He}^{+} \mathrm{e}^{-}$search to $50.6 \mathrm{pb}^{-1}$ and $52.3 \mathrm{pb}^{-1}$ respectively.

\section{Common features for all channels}

\subsection{Particle selection}

In all analyses, charged particles are selected if their momentum is greater than $100 \mathrm{MeV} / \mathrm{c}$ and if they originate from the interaction region (within $10 \mathrm{~cm}$ along the beam direction and within $4 \mathrm{~cm}$ in the transverse plane). Neutral particles are defined either as energy clusters in the calorimeters not associated to charged particle tracks, or as reconstructed vertices of photon conversions, interactions of neutral hadrons or decays of neutral particles in the tracking volume. All neutral clusters of energy greater than $200 \mathrm{MeV}$ (electromagnetic) or $500 \mathrm{MeV}$ (hadronic) are used; clusters in the range $100-500 \mathrm{MeV}$ are considered with specific quality criteria in some analyses. The $\pi^{ \pm}$mass is used for all charged particles except identified leptons, while zero mass is used for electromagnetic clusters and the $\mathrm{K}^{0}$ mass is assigned to hadronic clusters.

\section{2 b-quark identification}

The method of separation of b quarks from other flavours is described in [15], where the various differences between B-hadrons and other particles are accumulated in a single variable, hereafter denoted $x_{\mathrm{b}}$ for an event and $x_{\mathrm{b}}^{i}$ for jet $i$. One input to the combined variable is the probability that all tracks in a group originate from the interaction point. This is called $P_{\mathrm{E}}$ for all tracks in a jet or event, and $P_{\mathrm{E}}^{+}$for those particles with a positive lifetime-sign, and approaches zero for b quarks. $x_{\mathrm{b}}$ combines $P_{\mathrm{E}}^{+}$with information from secondary vertices (the mass computed from the particles assigned to the secondary vertex, the rapidity of those particles, and the fraction of the jet momentum carried by them) by computing the ratio of likelihood distributions for the signal and for the background. The procedure is calibrated on events recorded at the $\mathrm{Z}$ resonance, where the jets have similar characteristics to those from $\mathrm{ZZ}$ or $\mathrm{ZH}$. The suppression of various backgrounds is illustrated in Fig. 1. The combined b-tagging provides a substantial gain in rejection of background as compared to the b-tagging using only impact parameter information $[16,1]$. 

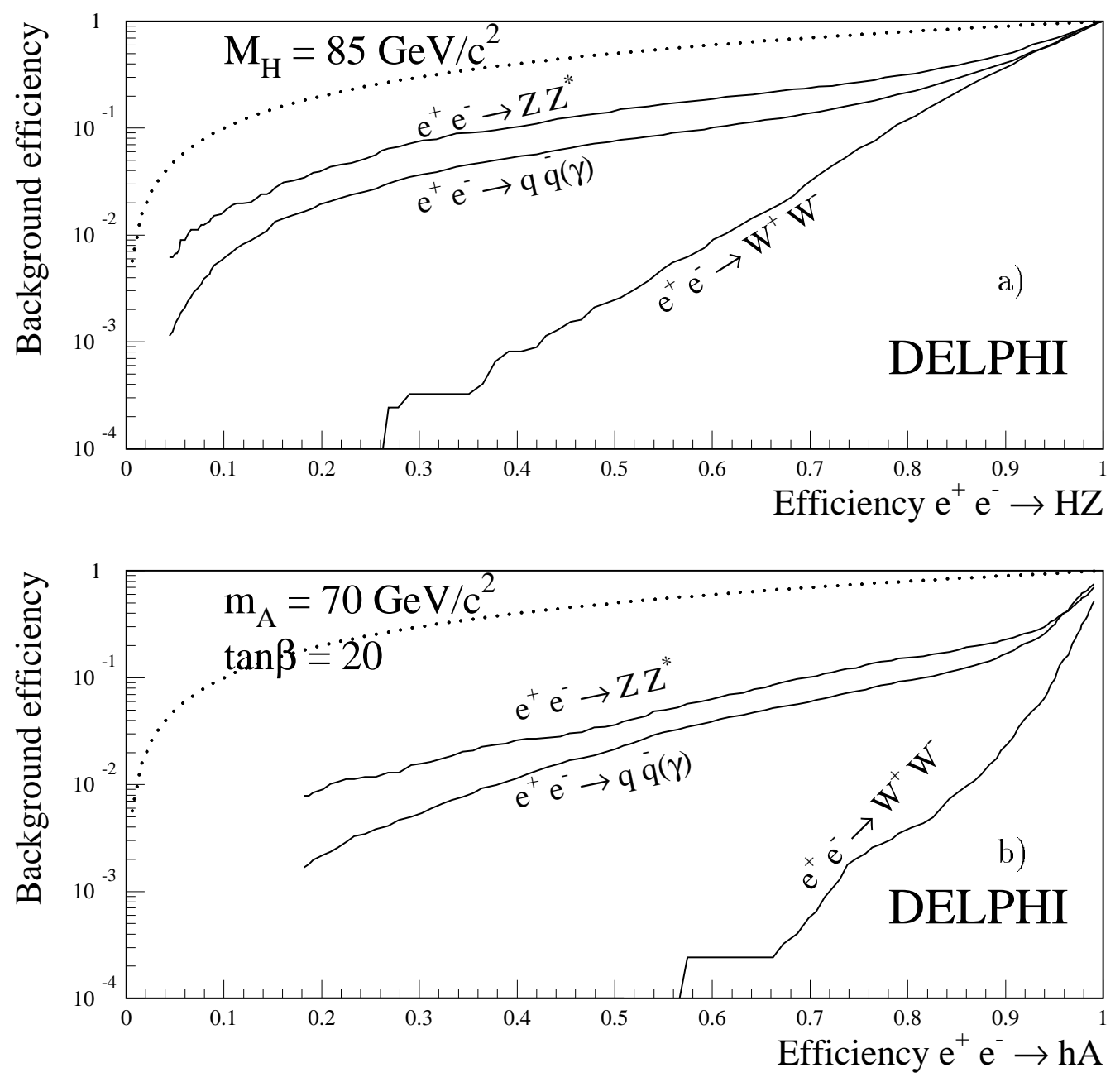

Figure 1: Performance of the combined b-tagging at $\sqrt{s}=183 \mathrm{GeV}$ : fraction of background events remaining after a b-tagging requirement as a function of the corresponding signal efficiency. Full curves are shown for simulated hadronic four-jet events from $\mathrm{W}^{+} \mathrm{W}^{-}$ pairs, ZZ pairs and $q \bar{q}(\gamma)$ background events, as a function of the efficiency on simulated four-jet events from a) $\mathrm{ZH}$ signal and b) hA signal. The rejection of $\mathrm{W}^{+} \mathrm{W}^{-}$pairs is easiest, because these contain very few $b$ quarks. The dotted curve shows the efficiency for the signal, for reference. The background is smaller in the channels because these events contain more b-quarks. 


\subsection{Constrained fits}

In all channels a constrained fit [17] is performed to extract the Higgs mass, and often to reject background processes as well. If only total energy and momentum conservation are imposed then the fit is referred to as ' 4 -C', while some fits require the $\mathrm{Z}$ mass as well, either as a fixed value, or taking into account the Breit-Wigner shape of the $\mathrm{Z}$ resonance. In both cases such fits are referred to as ' 5 -C'. The hadronic system assigned to the Higgs is forced to two jets for the fits. The treatment of an object in the fit depends upon whether it is an electron, $\mu, \tau$ or hadronic jet candidate.

\subsection{Confidence levels and the selection criteria determination}

Selection criteria have been tuned in order to optimize the expected exclusion of the Higgs signals using confidence level computations. The confidence with which the signal hypothesis can be rejected, $C L_{s}$, is calculated using the likelihood ratio technique [18]. This means that we find $\mathcal{L}$, the ratio of the likelihood of the observed candidates assuming signal plus background to that found using the background only hypothesis:

$$
\mathcal{L}=e^{-S} \Pi_{i} \frac{s_{i}+b_{i}}{b_{i}}
$$

where $S$ is the total expected signal, and $s_{i}$ and $b_{i}$ are the signal and background densities in mass for each candidate $i$. This therefore includes the information obtained from the reconstructed masses of any candidates. Combination of channels is achieved by simply allowing the appropriate event density for each channel.

We calculate $C L_{b}$ and $C L_{s b}$ :

$$
\begin{gathered}
C L_{b}=\mathcal{P}\left(\mathcal{L}^{\text {background }}<\mathcal{L}^{\text {obs }}\right) \\
C L_{s b}=\mathcal{P}\left(\mathcal{L}^{\text {signal+background }}<\mathcal{L}^{\text {obs }}\right) .
\end{gathered}
$$

i.e. the fraction of gedanken experiments with background only or signal and background which give smaller values of $\mathcal{L}$ than that observed. We then make the conservative step of defining $C L_{s}$ as the ratio of these probabilities:

$$
C L_{s}=\frac{C L_{s b}}{C L_{b}}
$$

The required gedanken experiments are made by Monte Carlo simulation, using the background and signal mass distributions estimated herein. The signal mass distributions which are simulated at $5 \mathrm{GeV}$ intervals are interpolated for intermediate values [19]. The signal efficiencies are fitted with simple functions, to obtain the expected signal rate at any given mass.

Errors on the background rate and signal efficiency are included by taking values for each gedanken experiment which are chosen from the distribution allowed by the error. The effect is rather small, as will be seen in Sect. 9.3.

\subsection{Analysis optimization}

We wish to set the efficiencies of each channel so that the sensitivity of the combination is maximised. The choice of the final working point for each analysis is made with an automated optimization procedure, using the expected (mean) confidence $\left\langle C L_{s}\right\rangle$ in the signal hypothesis if there is no signal taking into account all $\mathrm{ZH}$ (or hA) channels. The 
minimum value of this is found by varying the working points of all channels. This is done only once, using a Higgs mass of $85 \mathrm{GeV} / c^{2}$. More details are given in Sect. 4.4.

Finally, some of the analyses, such as discriminant analyses or neural networks, use selection techniques internally where the shapes of the Monte Carlo samples are used to generate selection criteria. With such techniques there is a danger of over-training the selection criteria by using random fluctuations in the simulated data sets. This is controlled by splitting the simulation into two halves with one half used to train the analysis and the other to test the outcome.

\section{$5 \quad$ Higgs boson searches in events with jets and elec- trons or muons}

The $\mathrm{H} \mu^{+} \mu^{-}$and $\mathrm{He}^{+} \mathrm{e}^{-}$channels together represent $6.7 \%$ of the $\mathrm{ZH}$ final states. The experimental signature is a pair of jets recoiling against a pair of high momentum and isolated leptons where the invariant mass of the lepton pair is close to the $\mathrm{Z}$ mass.

\subsection{Lepton identification and jet reconstruction}

Muon identification is provided primarily by the algorithm described in Ref. [13] which relies on the association of charged particle tracks to signals in the barrel and forward muon chambers. The same algorithm was also extended to the surrounding muon chambers. The longitudinal profile of the energy deposit in the hadron calorimeter is also used to improve the efficiency of muon identification. Since 1997, detailed information about the shape of the hadronic shower provided by the new HCAL cathode readout has been included in the muon identification algorithm. The performance of the muon identification at $183 \mathrm{GeV}$ has been evaluated using $\mathrm{Z} \rightarrow \mu^{+} \mu^{-}(\gamma)$ and $\mathrm{Z} \rightarrow \tau^{+} \tau^{-}(\gamma)$ simulated events. The efficiency corresponds to the fraction of charged particles in $\mu^{+} \mu^{-}(\gamma)$ events, with momentum above $10 \mathrm{GeV} / c$, which are identified as muons by the algorithm. The misidentification probability is defined as the fraction of kaons and pions in the same momentum range coming from simulated $\tau^{+} \tau^{-}(\gamma)$ events which are misidentified as muons by the algorithm. Different possible working points of the algorithm are presented in Fig. 2 a).

A specific electron identification algorithm was developed for Higgs boson searches with the emphasis on efficiency rather than purity, as electrons in the $\mathrm{ZH}$ channel are expected to be isolated. Electrons are identified as charged particle tracks with an energy deposit above $3 \mathrm{GeV}$ in the electromagnetic calorimeter, below $1.6 \mathrm{GeV}$ beyond the first layer of the hadronic calorimeter, and with a ratio of calorimeter energy to momentum from tracking above 0.3. A charged particle track pointing to an insensitive calorimeter region is also accepted provided it is not identified as a muon and is either associated with a hit in the hermeticity taggers or has an energy loss in the TPC in agreement with that expected for an electron. In both cases, electrons from gamma conversion at large radius are rejected by requiring the track reconstruction to include points in the TPC or, if the particle passes between the modules of the TPC, points both in the microvertex and inner detectors. In the forward region $\left(\theta<43^{\circ}\right.$ and $\left.\theta>137^{\circ}\right)$ the track is only required to include points in the microvertex detector.

The energy of an electron candidate in the barrel region is defined by a weighted average of the calorimeter energy and the momentum given by the tracking detectors (using weights tuned on real and simulated Bhabha events) except if the track points 
to an insensitive region of the calorimeters, in which case only the momentum is used. The electron identification efficiency, measured on a sample of simulated $\mathrm{He}^{+} \mathrm{e}^{-}$events, is $94 \%$ within the acceptance of the tracking system. The price of such a high efficiency is a probability of misidentifying a pion as an electron of $16 \%$. When accepting only tracks linked to an electromagnetic shower the efficiency is $83 \%$ and the misidentification probability is $13 \%$. A cross-check of the data, based on the selection of Bhabha events where one electron is used as a trigger and the other as a member of the test sample, has confirmed this efficiency.

\subsection{Muon channel}

Events are required to have at least four charged particles and a total energy from charged particles above $0.30 \sqrt{s}$. The total energy in the barrel electromagnetic calorimeter must be less than $100 \mathrm{GeV}$ and at least two charged particles must be reconstructed in the barrel region. This rejects $99.9 \%$ of Bhabha and $\gamma \gamma$ events. Among the charged particles, two of them must have opposite charges and momenta greater than $10 \mathrm{GeV} / c$. In order to take into account possible final-state radiation, any photon collinear with a muon candidate and having an energy larger than $2.5 \mathrm{GeV}$ has its energy added to the muon momentum and the corresponding error is modified accordingly. In the case of low multiplicity events (charged multiplicity below ten) the acollinearity of the selected pair must be greater than $2^{\circ}$ to remove $\mu \mu(\gamma)$ and $\tau \tau(\gamma)$ events. After this preselection, the expected background is dominated by $\mathrm{q} \overline{\mathrm{q}}(\gamma)$ and $\mathrm{W}^{+} \mathrm{W}^{-}$events.

Each pair of particles satisfying these criteria is then considered in turn. The momentum (including any collinear radiation) of the faster particle of the pair must be greater than $39 \mathrm{GeV} / c$, and the slower one above $15 \mathrm{GeV} / c$. The opening angle between the particles must be greater than $109^{\circ}$. Muon identification is required for both particles of the pair. A search for jets is then performed in the system recoiling from the muon pair, using the DURHAM algorithm [20] with a resolution parameter of 0.12. Events are selected if at least two jets are reconstructed and if there is at least one charged particle in the second most energetic jet. This last criterion is introduced to avoid fake jets caused by noise or by photons showering in the detector. Finally, the isolation angles to the closest jet, $\psi^{\text {jet }}$ of the two muon candidates are required to be more than $15^{\circ}$ and $9^{\circ}$. A 5 - C kinematic fit is then performed, which, in addition to total energy and momentum conservation, requires the mass of the muon pair to be consistent with $m_{\mathrm{Z}}$, taking into account the Breit-Wigner shape of the $\mathrm{Z}$ resonance. Events are only kept if the fit probability is higher than $10^{-6}$. After the fit, the dominant background is made up of ZZ events, with one $\mathrm{Z}$ decaying into $\mu^{+} \mu^{-}$and the other into hadrons. The b-tagging procedure is then used to discriminate the light quark decays of the $\mathrm{Z}$ from a Higgs boson decay into $\mathrm{b} \bar{b}$. In order not to lose efficiency in signal mass zones far from $\mathrm{Z}$ mass, a b-tagging cut is applied only if the measured recoiling mass to the di-muon is above $84 \mathrm{GeV} / c^{2}$. A loose b-tag probability cut is used; it is the minimum value of $P_{\mathrm{E}}^{+}$and $P_{\mathrm{E}}$ for the event being required to be below 0.10 . Events are kept if the above criteria are fulfilled for at least one muon pair.

The values of the above selection cuts were obtained by a procedure which optimizes them one after another. The value of each selection cut is varied over a given range and only those corresponding to the best efficiency for a given background are retained. The optimization was performed on half of the simulation samples and the optimal sets of cuts were applied to the remaining simulation samples to define the final efficiencies and backgrounds. All Higgs boson masses from 60 to $95 \mathrm{GeV} / c^{2}$ were taken into account 
in the optimization. The curve of efficiency as a function of background number of events, obtained after optimization, is presented in Fig. $2 \mathrm{~b}$ ). The final working point is determined by the global optimization procedure at $m_{\mathrm{H}}=85 \mathrm{GeV} / c^{2}$ as described in Sect. 4.5.
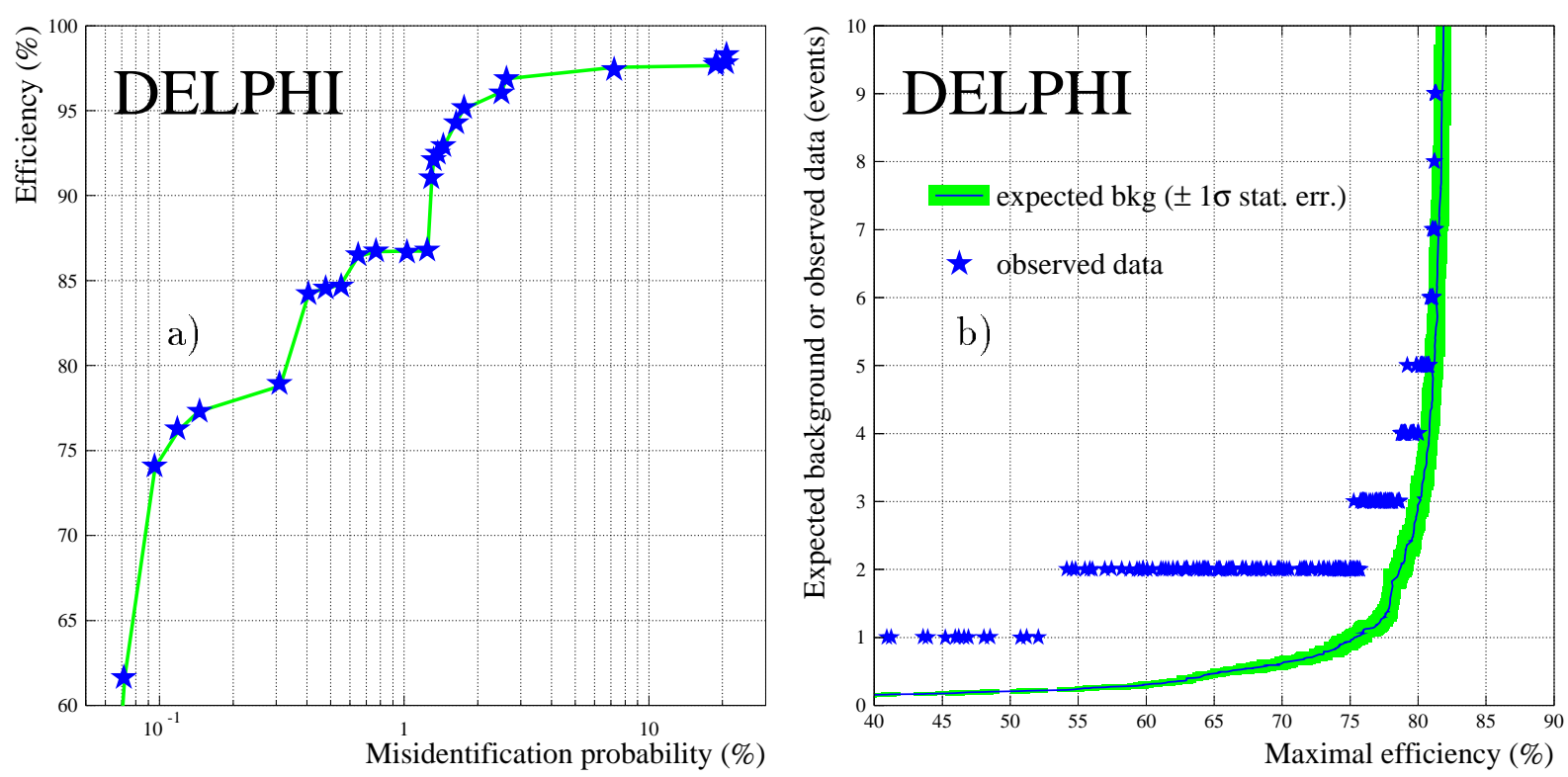

Figure 2: $\mathrm{H} \mu^{+} \mu^{-}$channel: a) the performance of the muon identification. Efficiency is given for muons with momentum above $10 \mathrm{GeV} / c$ coming from $\mathrm{Z} \rightarrow \mu^{+} \mu^{-}(\gamma)$ events at $183 \mathrm{GeV}$. The probability of misidentifying a charged hadron as a muon is measured using charged kaons and pions coming from $Z \rightarrow \tau^{+} \tau^{-}(\gamma)$ events. The kinks correspond to changes in the way the information is combined. b) Expected background as a function of the maximal efficiency obtained at each background level for a Higgs signal of $m_{\mathrm{H}}=85 \mathrm{GeV} / c^{2}$. Statistical errors are indicated by the narrow band around the curve. Stars correspond to the number of observed data events for a given working point. Notice that the working point definition involves optimizing several cuts simultaneously, and not a cut on just one variable. Thus the events selected at any given efficiency need not be a subset of those at higher efficiencies, and this explains the overlap when 4 or 5 events are observed.

Table 2 details the effect of the selection cuts on data and simulated samples of background and signal events. The agreement of simulation with data is good. This can also be seen in Fig. 3, which shows the distributions of the momenta and isolation angles of the high-momentum particles at the preselection level. At the end of the analysis, the expected background comes mainly from ZZ events, and amounts to $0.49 \pm 0.06$ (stat.) \pm 0.17 (syst.) events. The mass resolution of the selected events is about $2 \mathrm{GeV} / c^{2}[1]$.

Because of the finite size of the Monte Carlo data samples, zero quantities appear in the background estimates at different levels of the selection. To check that these zeroes are realistic, a second estimator of the background was used. Muon identification was removed from the selection and, for each background process, the background after the remaining selection cuts was multiplied by the efficiency of the muon identification step, taken from Table 2. This leads to a total background of $0.36 \pm 0.06$ (stat.) event, in good 

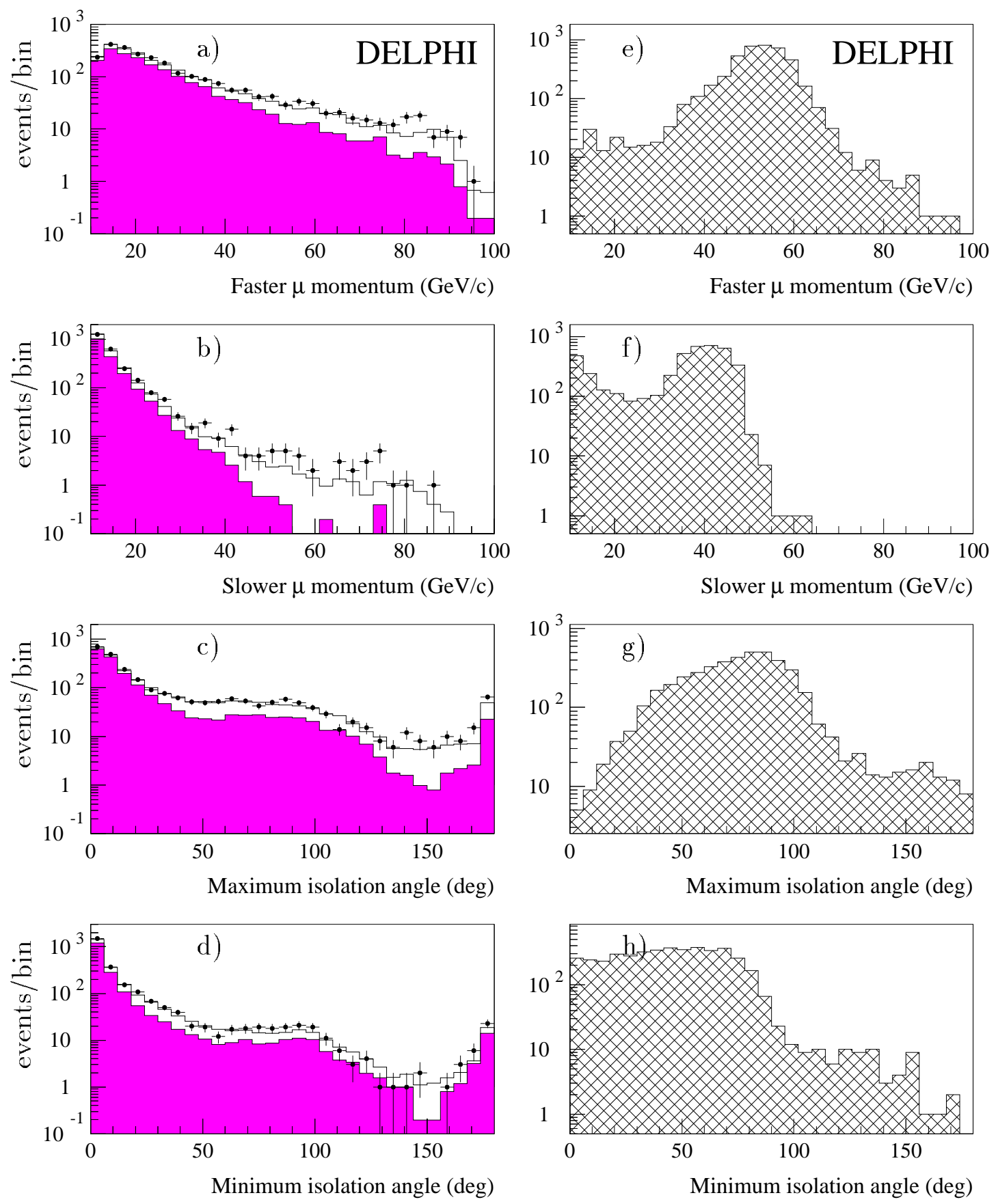

Figure 3: $\mathrm{H} \mu^{+} \mu^{-}$channel: distributions of some analysis variables as described in the text. Plots a) to d) show a comparison between $183 \mathrm{GeV}$ data (points) and simulated background events (solid line) normalized to the experimental luminosity. The shaded area represents the contribution of the dominant $q \bar{q}(\gamma)$ background. Plots e) to h) show the (unnormalized) expected distributions for a Higgs boson of $85 \mathrm{GeV} / c^{2}$. 


\begin{tabular}{|c|c|c|c|c|c|c|c|c|c|}
\hline \multirow[t]{2}{*}{ Selection } & \multirow{2}{*}{ Data } & \multirow{2}{*}{$\begin{array}{c}\text { Total } \\
\text { background }\end{array}$} & \multirow[t]{2}{*}{$q \bar{q}(\gamma)$} & \multicolumn{3}{|c|}{4 fermion } & \multirow[t]{2}{*}{$1^{+} 1^{-}(\gamma)$} & \multirow[t]{2}{*}{$\gamma \gamma$} & \multirow{2}{*}{$\begin{array}{l}\mathrm{H} \mu^{+} \mu^{-} \\
\varepsilon(\%)\end{array}$} \\
\hline & & & & $\overline{\mathrm{ZZ}}$ & $\mathrm{W}^{+} \mathrm{W}^{-}$ & Zee /We $\nu$ & & & \\
\hline Preselection & 2461 & $2387 \pm 17$ & 1838 & 26.7 & 350 & 45.6 & 78.8 & 47.6 & 90.6 \\
\hline \multicolumn{10}{|l|}{ Momenta and } \\
\hline opening angle & 224 & $196 \pm 5$ & 116 & 5.40 & 39.8 & 5.02 & 24.4 & 5.49 & 85.5 \\
\hline$\mu^{+} \mu^{-}$selection & 9 & $7.44 \pm 0.64$ & 0.59 & 1.94 & 2.14 & 0.72 & 1.75 & 0.30 & 80.2 \\
\hline Jets & 5 & $3.90 \pm 0.45$ & 0.59 & 1.16 & 2.00 & 0 & 0.07 & 0.07 & 77.5 \\
\hline Isolation & 4 & $1.56 \pm 0.15$ & 0 & 1.04 & 0.52 & 0 & 0 & 0 & 75.7 \\
\hline 5-C fit & 3 & $0.94 \pm 0.07$ & 0 & 0.90 & 0.04 & 0 & 0 & 0 & 72.8 \\
\hline b-tagging & 2 & $0.49 \pm 0.06$ & 0 & 0.45 & 0.04 & 0 & 0 & 0 & 65.0 \\
\hline
\end{tabular}

Table 2: $\mathrm{H} \mu^{+} \mu^{-}$channel: effect of the selection cuts on data, simulated background and simulated signal events at $\sqrt{s}=183 \mathrm{GeV}$ with $m_{\mathrm{H}}=85 \mathrm{GeV} / c^{2}$. Efficiencies are given for the signal simulation.

agreement with the previous one. The contributions from $q \bar{q}(\gamma)$ and $W^{+} W^{-}$background are $0.004 \pm 0.003$ (stat.) and $0.024 \pm 0.007$ (stat.) events respectively, confirming that the ZZ background dominates the sample.

Two events are left in the data after selection, compared to 0.49 expected from the simulation. The first one has two muons of momenta 60 and $35 \mathrm{GeV} / c$ with an invariant mass of $89.5 \mathrm{GeV} / c^{2}$ and a recoiling mass of $82.9 \mathrm{GeV} / c^{2}$ after the kinematic fit. This event shows no b-quark signature and no secondary vertex was found. A longitudinal view of the event is shown in Fig. 4. The other event has two clearly identified muons in the barrel muon chamber. A dimuon mass of $89.9 \mathrm{GeV} / c^{2}$ and a recoiling mass of $89.9 \mathrm{GeV} / c^{2}$ are obtained after kinematic fit. This event has a significant b-tag, and 4 tracks are found to form a secondary vertex. A transverse view of the event is shown in Fig. 5. The main characteristics of the events are summarized in Table 3.

\begin{tabular}{ccccccc}
\hline $\begin{array}{c}\mathrm{p}_{\mu 1} \\
(\mathrm{GeV} / c)\end{array}$ & $\begin{array}{c}\mathrm{p}_{\mu 2} \\
(\mathrm{GeV} / c)\end{array}$ & $\begin{array}{c}\psi_{\mu 1}^{\text {jet }}\left({ }^{\circ}\right) \\
\left(^{\text {jet }}\right.\end{array}$ & $\begin{array}{c}{ }^{\text {jet }} \\
\left(\mathrm{GeV} / c^{2}\right)\end{array}$ & $\begin{array}{c}\mathrm{M}_{\mu \mu}^{\text {fit }} \\
\left(\mathrm{GeV} / c^{2}\right)\end{array}$ & $\begin{array}{c}\mathrm{M}_{\text {rec }}^{\text {fit }} \\
\min \left(P_{\mathrm{E}}, P_{\mathrm{E}}^{+}\right)\end{array}$ \\
\hline 60.0 & 35.2 & 29.4 & 55.4 & 89.52 & 82.9 & 0.12 \\
49.2 & 42.1 & 80.6 & 84.9 & 89.9 & 89.9 & $5.8 \cdot 10^{-14}$ \\
\hline
\end{tabular}

Table 3: Characteristics of the selected events in the $\mathrm{H} \mu^{+} \mu^{-}$channel: the muon momenta and angles of isolation from closest jet $(\psi)$, dimuon and recoiling mass after kinematic fit and b-tagging probability are quoted. The first event has a low mass, and so the b-tagging requirement is not made.

The signal efficiencies for different Higgs boson masses are given in Table 4 and shown graphically in Fig. 29. The decrease of the efficiency at low mass is due to the larger boost of the Higgs boson which makes the requirements on the number of jets less efficient. The decrease of the efficiency at high mass is, on one hand, due to b-tagging which is only applied to recoiling masses close to $m_{\mathrm{Z}}$, and, on the other hand, to the kinematic fit which 


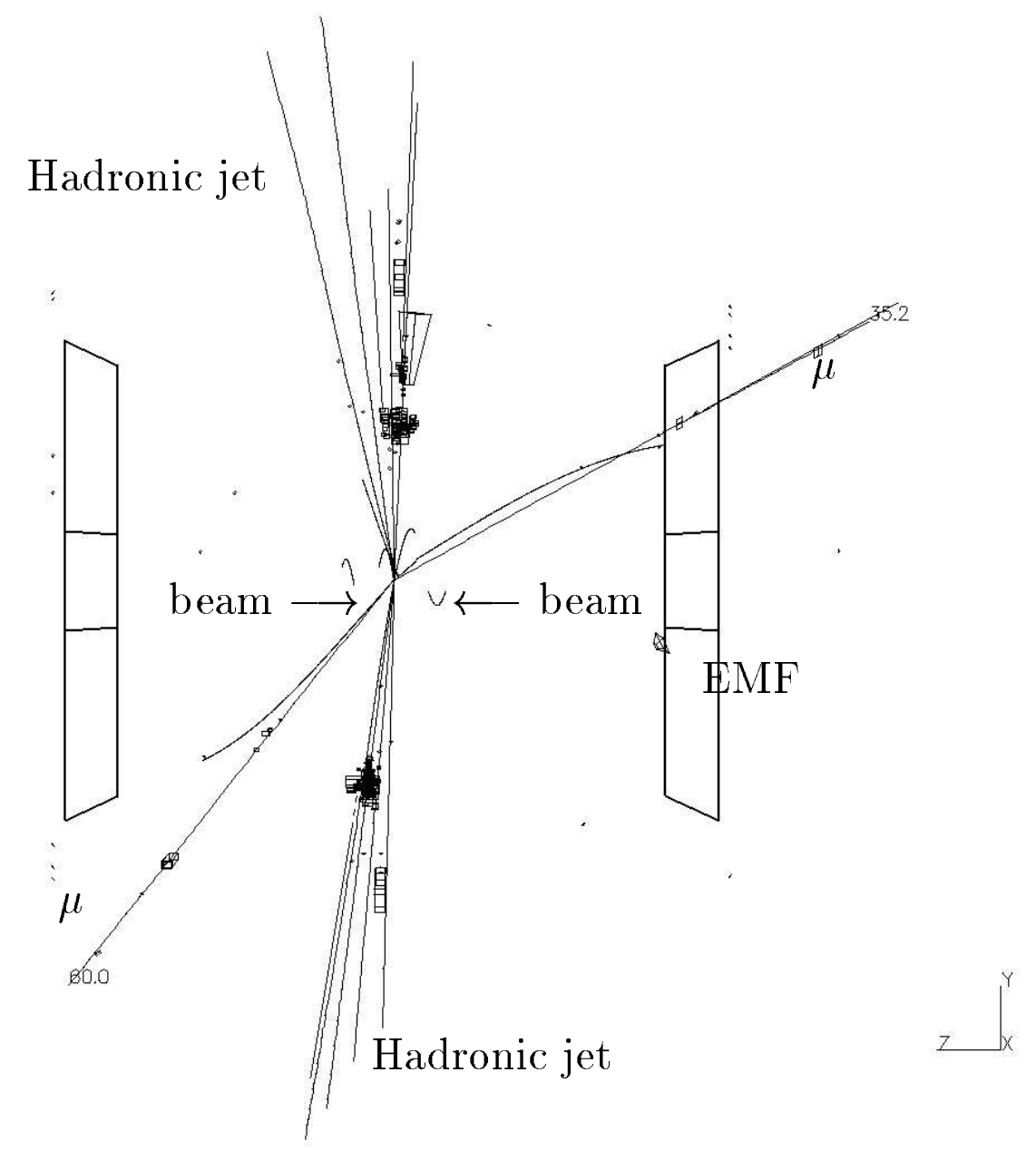

Figure 4: $\mathrm{H} \mu^{+} \mu^{-}$channel: longitudinal view of the event with little evidence for b quarks. The reconstructed charged particle tracks, energy deposits and the geometry of the forward electromagnetic calorimeters (EMF) are displayed. The two muon candidates are the tracks isolated from the jets. 


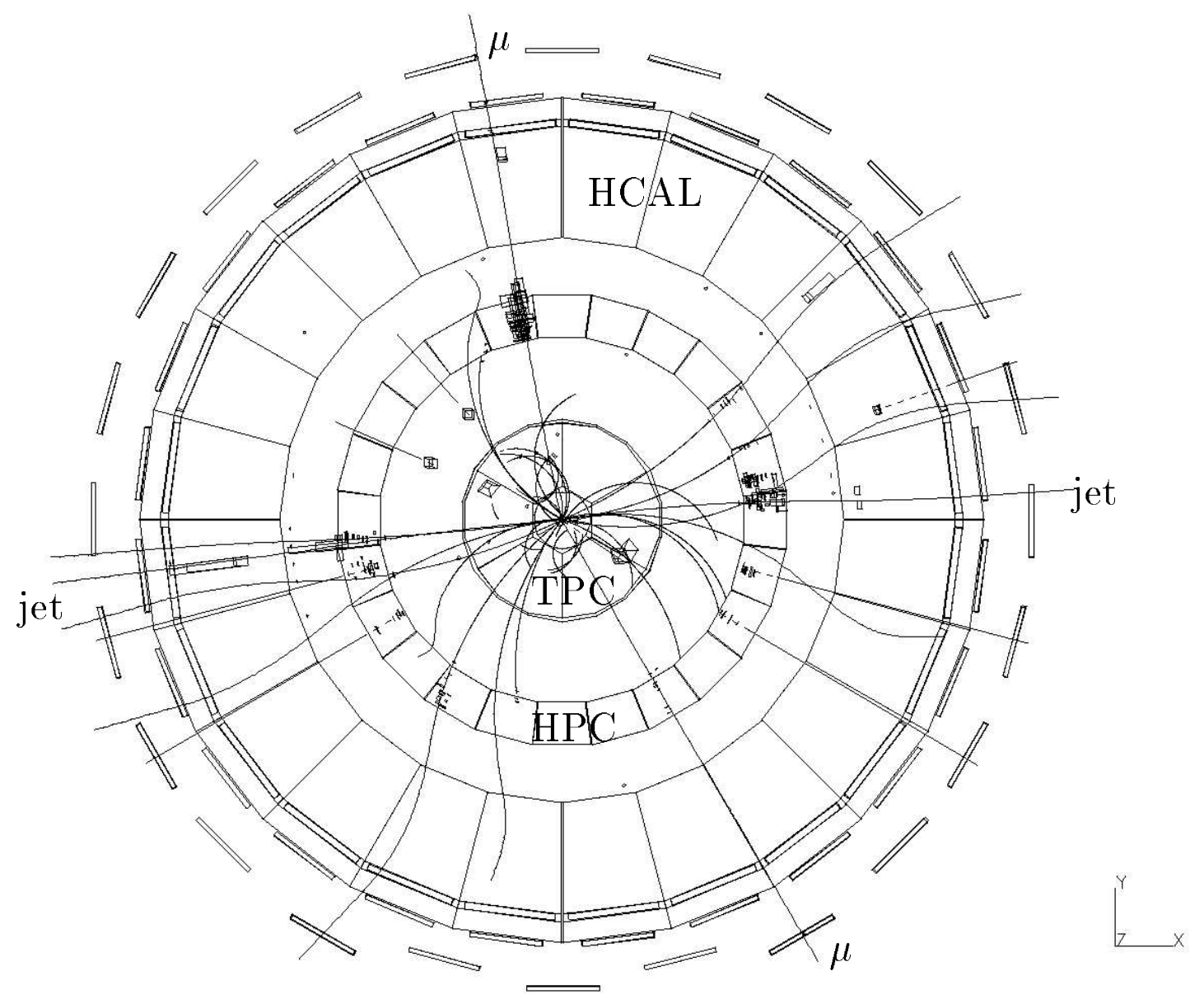

Figure 5: $\mathrm{H} \mu^{+} \mu^{-}$channel: transverse view of the event with high b-quark content. 
rejects events where the $\mathrm{Z}$ boson is off shell, which becomes more likely as the mass of the Higgs rises and the available energy shrinks.

\begin{tabular}{cccc}
\hline$m_{\mathrm{H}}\left(\mathrm{GeV} / c^{2}\right)$ & Efficiency $(\%)$ & $m_{\mathrm{H}}\left(\mathrm{GeV} / c^{2}\right)$ & Efficiency $(\%)$ \\
\hline 60 & $61.3 \pm 1.1_{-0.9}^{+0.9}$ & 80 & $70.1 \pm 0.9{ }_{-1.0}^{+0.7}$ \\
65 & $63.5 \pm 1.1_{-1.1}^{+0.8}$ & 85 & $65.0 \pm 0.7_{-0.8}^{+0.7}$ \\
70 & $66.3 \pm 0.7_{-0.9}^{+0.8}$ & 90 & $60.7 \pm 0.7_{-0.8}^{+0.7}$ \\
75 & $68.1 \pm 1.0_{-0.9}^{+0.8}$ & 95 & $54.2 \pm 1.0_{-0.8}^{+0.7}$ \\
\hline
\end{tabular}

Table 4: $\mathrm{H} \mu^{+} \mu^{-}$channel: efficiency of the Higgs boson selection at $\sqrt{s}=183 \mathrm{GeV}$ as a function of the mass of the Higgs boson. The first uncertainty quoted is statistical, the second is systematic.

A first source of systematic effects is the imperfect simulation of the detector response. The agreement of real and simulated distributions for the analysis variables was checked at the preselection level, and no crucial discrepancy was found.

For each continuous variable used in the optimization, the mean values in the real data and the simulated samples are compared. The systematic uncertainties are then estimated by varying the cuts on each of these variables by the difference in the mean. The corresponding changes in the efficiencies and background estimates obtained for each variable are finally added quadratically. To take into account possible differences between data and simulation in the muon identification, a relative $1 \%$ uncertainty [13] has been added quadratically to get the final systematic uncertainties on the efficiencies, which are given in Table 4.

The errors on background estimates given by the previous method are found to be negligible in comparison to the effect of a possible incorrect simulation of standard processes. The simulated distributions for the analysis variables of four-fermion processes obtained with PYTHIA and EXCALIBUR have been compared at each step of the selection. Good agreement is observed for all variables except dimuon masses and hence for the fit probability. After the isolation cut, the total background predicted by PYTHIA is $1.56 \pm 0.15$ (stat.), close to the $1.78 \pm 0.23$ (stat.) given by EXCALIBUR. This agreement has been checked for different sets of cuts and is always correct before kinematic fit variable cuts. The discrepancy observed in the dimuon mass distribution, which is broader in PYTHIA samples than in EXCALIBUR ones, could be due to the lack of final-state radiation simulation in EXCALIBUR. This affects the kinematic fit procedure and leads to a final background predicted by EXCALIBUR of $0.65 \pm 0.11$ (stat.), one third larger than the PYTHIA estimate. To take this difference into account, a systematic uncertainty of \pm 0.17 has finally been given to the expected background.

\subsection{Electron channel}

Candidates for $\mathrm{e}^{+} \mathrm{e}^{-} \rightarrow \mathrm{He}^{+} \mathrm{e}^{-}$are preselected by requiring five or more charged particles and a total energy above $0.12 \sqrt{s}$. Among the charged particles of the event, two are required to have a momentum above $10 \mathrm{GeV} / c$ and must either be associated with a shower in the electromagnetic calorimeter or point to an insensitive calorimeter region. It is also required that the sum of the energies of other charged particles within a cone of $5^{\circ}$ around either electron be less than $8 \mathrm{GeV}$. Furthermore, the sum of the energy deposits in the electromagnetic calorimeters from the two electrons has to be above $10 \mathrm{GeV}$. The 
LUCLUS algorithm [21], with the resolution parameter set to $4 \mathrm{GeV} / c$, is applied to the system recoiling from the electron pair. Events must have at least two reconstructed jets with the second most energetic jet containing at least two charged particles.

All pairs of particles satisfying the above criteria are then considered as electron candidates. Both particles must satisfy the electron identification described above, with the restriction that the two particles are not allowed to point simultaneously to insensitive calorimeter regions. Events are kept if at least one pair of oppositely charged electron candidates is found. If the higher energy electron candidate is over $65 \mathrm{GeV}$, and is at less than $25^{\circ}$ to the beam axis, it is rejected as a Bhabha candidate. The energy of the slower (faster) electron is required to be above $15(32) \mathrm{GeV}$. Global 5-C kinematic fits are performed, with the $\mathrm{e}^{+} \mathrm{e}^{-}$mass fixed to a range of values between 60 and $120 \mathrm{GeV} / c^{2}$. A combined variable is defined which is the product of the $\chi^{2}$ probability of the fit times the probability (found using all simulated $\mathrm{He}^{+} \mathrm{e}^{-}$samples) that an $\mathrm{He}^{+} \mathrm{e}^{-}$event should have the difference between the mass of the $\mathrm{e}^{+} \mathrm{e}^{-}$system and $m_{\mathrm{Z}}$ at least as large as that observed. This quantity is used as an estimator of the goodness of the fit, and the recoil mass corresponding to its highest value is taken as the estimator of the Higgs mass. Events with a probability product below $10^{-22}$ are rejected.

Electron isolation angles with respect to the closest jet are required to be larger than $20^{\circ}$ for the more isolated electron and more than $10^{\circ}$ for the other. As the search is restricted to high mass Higgs bosons, the mass of the recoiling system, as given by the kinematic fit, is required to be above $50 \mathrm{GeV} / c^{2}$. The final selection is an event b-tag, based on the minimum of $P_{\mathrm{E}}^{+}$and $P_{\mathrm{E}}$, in order to keep the main background source (i.e. $\mathrm{ZZ}$ production) at an acceptable level. Since the b-tagging selection concerns mainly the ZZ background, it is applied only for fitted recoil masses above $80 \mathrm{GeV} / c^{2}$. The value of the b-tagging selection is chosen by the global optimization procedure of Sect. 4.5 and set to $\min \left(P_{\mathrm{E}}, P_{\mathrm{E}}^{+}\right)<0.005$.

Figure 6 shows the distributions of the main analysis variables after preselection, for $183 \mathrm{GeV}$ data, simulated background and signal events at $85 \mathrm{GeV} / c^{2}$. The agreement between data and background expectation is good.

\begin{tabular}{|c|c|c|c|c|c|c|c|c|c|}
\hline \multirow[t]{2}{*}{ Selection } & \multirow[t]{2}{*}{ Data } & \multirow{2}{*}{$\begin{array}{c}\text { Total } \\
\text { background }\end{array}$} & \multirow[t]{2}{*}{$\mathrm{q} \overline{\mathrm{q}}(\gamma)$} & \multirow[t]{2}{*}{$11(\gamma)$} & \multicolumn{3}{|c|}{4 fermion } & \multirow{2}{*}{$\begin{array}{c}\gamma \gamma \rightarrow \\
\text { hadronic }\end{array}$} & \multirow{2}{*}{$\begin{array}{l}\mathrm{He}^{+} \mathrm{e}^{-} \\
\varepsilon(\%)\end{array}$} \\
\hline & & & & & $\mathrm{e}^{+} \mathrm{e}^{-} \mathrm{q} \overline{\mathrm{q}}$ & $\mathrm{e} \nu \mathrm{q} \overline{\mathrm{q}}$ & others & & \\
\hline Hadronic & 8611 & $9826 \pm 27$ & 5078.2 & 2214.5 & 19.2 & 144.8 & 666.4 & 1670.1 & 94.9 \\
\hline Preselection & 370 & $345.4 \pm 3.5$ & 263.8 & 2.17 & 4.72 & 25.16 & 36.55 & 12.99 & 71.7 \\
\hline $\mathrm{e}^{+} \mathrm{e}^{-} \mathrm{id}$ & 35 & $36.1 \pm 1.5$ & 15.7 & 0.29 & 3.25 & 8.79 & 5.43 & 2.61 & 60.5 \\
\hline e momenta & 6 & $7.85 \pm 0.59$ & 0.99 & 0.23 & 2.53 & 3.26 & 0.37 & 0.46 & 57.9 \\
\hline 5 -C fit $\chi^{2}$ & 4 & $4.48 \pm 0.41$ & 0.70 & 0.06 & 1.97 & 1.36 & 0.18 & 0.23 & 57.0 \\
\hline Isolation & 4 & $2.21 \pm 0.19$ & 0.13 & 0.06 & 1.87 & 0.15 & 0.01 & 0 & 54.2 \\
\hline Recoil mass & 2 & $1.54 \pm 0.16$ & 0.12 & 0 & 1.26 & 0.15 & 0.01 & 0 & 54.2 \\
\hline b-tagging & 1 & $0.68 \pm 0.12$ & 0.06 & 0 & 0.47 & 0.15 & 0 & 0 & 39.9 \\
\hline
\end{tabular}

Table 5: $\mathrm{He}^{+} \mathrm{e}^{-}$channel: the numbers of events passing each selection. Efficiencies are given for the signal with $m_{\mathrm{H}}=85 \mathrm{GeV} / c^{2}$ at $\sqrt{s}=183 \mathrm{GeV}$.

Table 5 shows the effect of the selection cuts on data, simulated background and signal events. Backgrounds not quoted in the table (e.g. two-photon processes leading to leptonic 

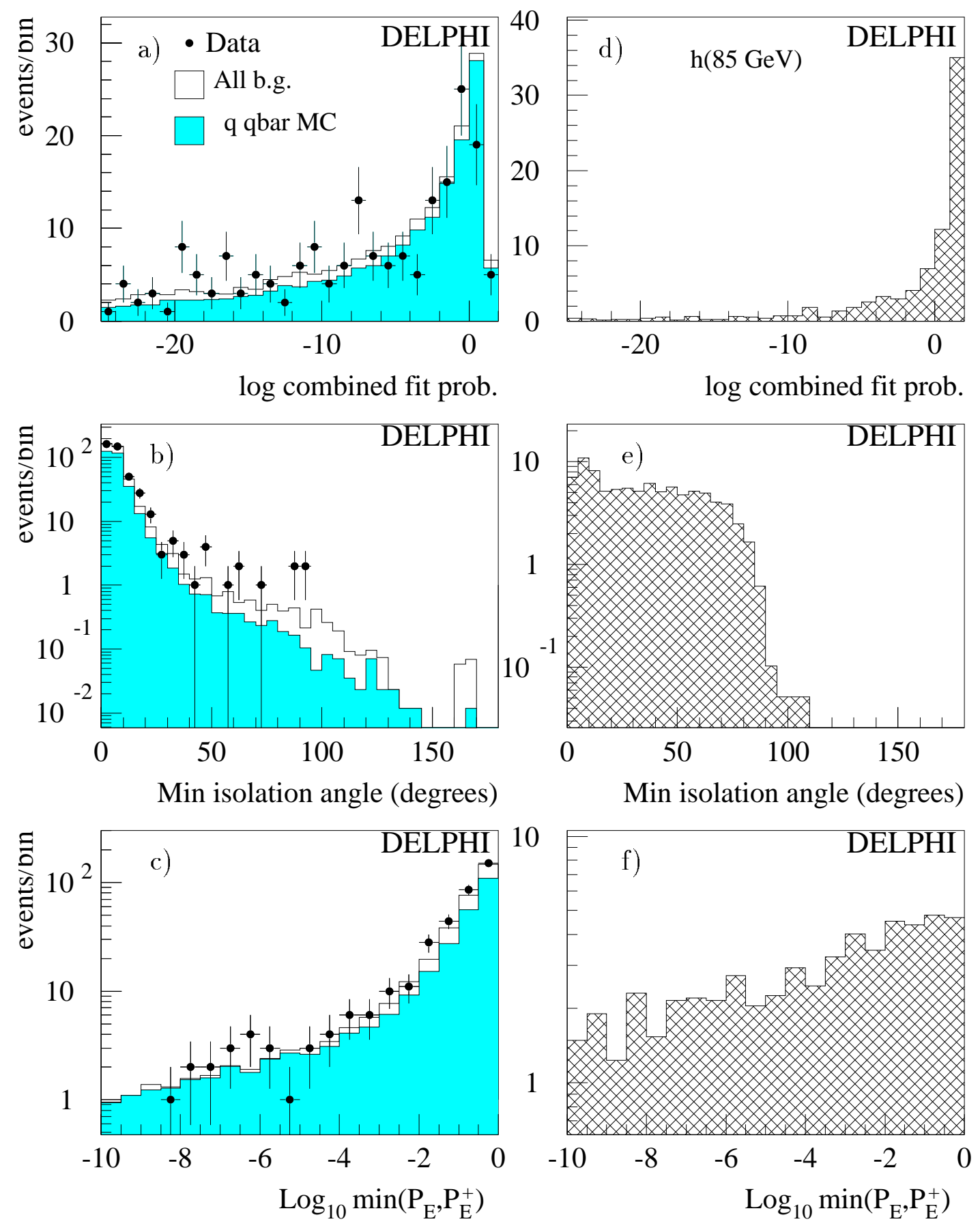

Figure 6: $\mathrm{He}^{+} \mathrm{e}^{-}$channel: distributions of some analysis variables as described in the text. Plots a) to c) show a comparison between $183 \mathrm{GeV}$ data (points) and simulated events (full line) normalized to the experimental luminosity. Plots d) to f) show the (unnormalized) expected distributions for an $85 \mathrm{GeV} / c^{2}$ Higgs boson after all cuts. 


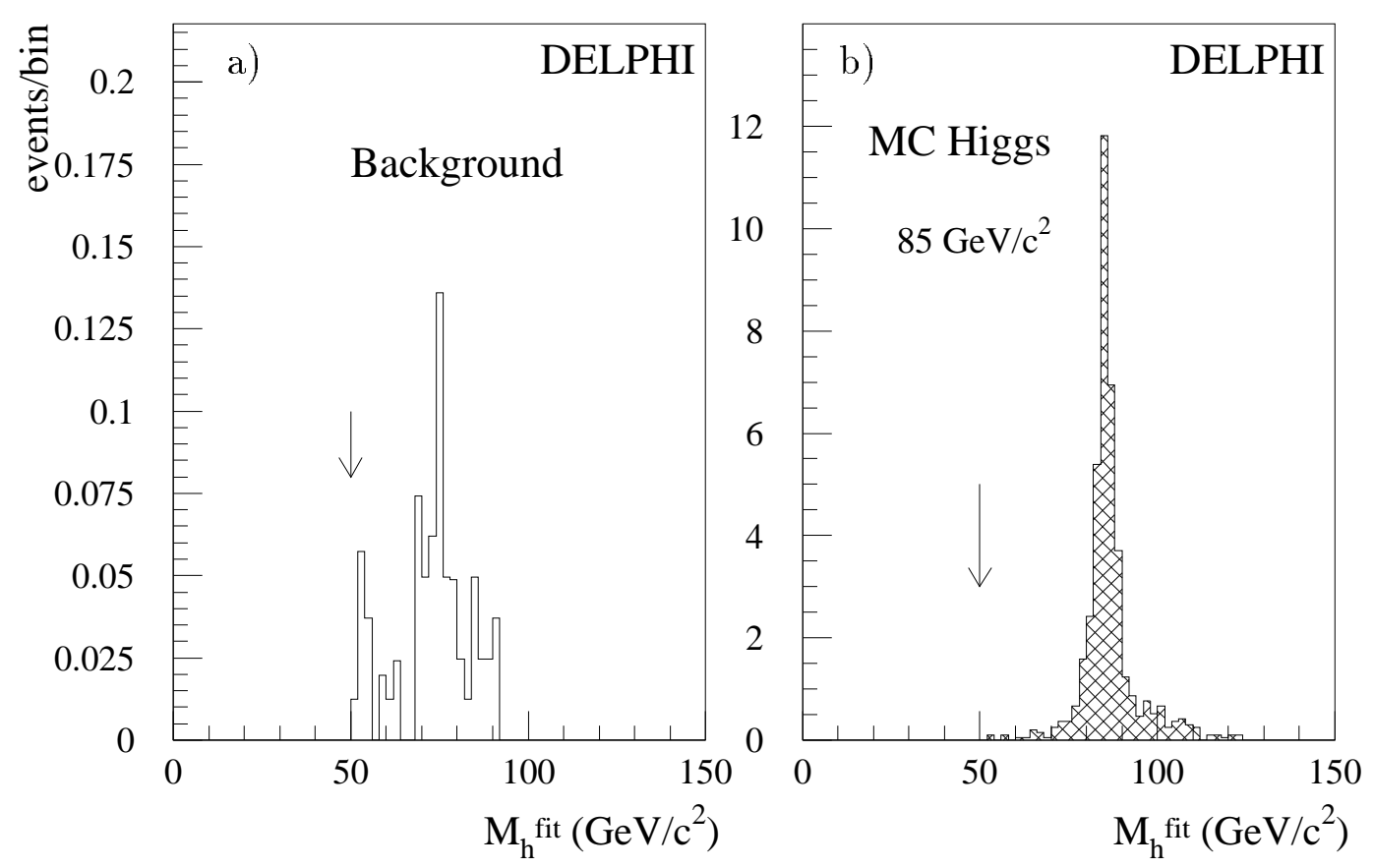

Figure 7: $\mathrm{He}^{+} \mathrm{e}^{-}$channel: the distribution of the fitted recoil masses at the end of the analysis for a) background and b) simulated $\mathrm{He}^{+} \mathrm{e}^{-}$events with $m_{\mathrm{H}}=85 \mathrm{GeV} / c^{2}$ at $183 \mathrm{GeV}$. The arrow indicates the cut at $50 \mathrm{GeV} / c^{2}$, which has already been applied.

final states) are negligible. The final background amounts to $0.68 \pm 0.12$ (stat.) ${ }_{-0.10}^{+0.09}$ (syst.) events, and is mainly due to $\mathrm{e}^{+} \mathrm{e}^{-} \mathrm{q} \overline{\mathrm{q}}$ events, with the $\mathrm{ZZ}$ process dominant. Fig. 7 illustrates the distribution of reconstructed Higgs boson masses as given by the kinematic fit in simulated background and signal events.

Table 6 and Fig. 29 show the selection efficiency for different masses at the working point selected by the global optimization.

\begin{tabular}{cccc}
\hline$m_{\mathrm{H}}\left(\mathrm{GeV} / c^{2}\right)$ & Efficiency $(\%)$ & $m_{\mathrm{H}}\left(\mathrm{GeV} / c^{2}\right)$ & Efficiency $(\%)$ \\
\hline 60 & $48.0 \pm 1.1_{-1.2}^{+0.4}$ & 80 & $43.1 \pm 1.1_{-4.0}^{+4.9}$ \\
65 & $49.9 \pm 1.1_{-0.7}^{+0.5}$ & 85 & $39.9 \pm 1.1_{-2.1}^{+3.1}$ \\
70 & $50.1 \pm 1.1_{-1.5}^{+0.8}$ & 90 & $38.2 \pm 1.1_{-1.8}^{+1.0}$ \\
75 & $47.5 \pm 1.1_{-2.8}^{+1.6}$ & 95 & $34.8 \pm 1.1_{-1.6}^{+1.2}$ \\
\hline
\end{tabular}

Table 6: $\mathrm{He}^{+} \mathrm{e}^{-}$channel: efficiency of the Higgs boson selection at $\sqrt{s}=183 \mathrm{GeV}$ as a function of the mass of the Higgs boson. The first uncertainty quoted is statistical, the second is systematic.

The systematic uncertainties have been evaluated by considering both the difference between data and simulation in the distributions of each analysis variable and the sensitivity of the selection criteria to such variables. The variables included in this procedure are the continuous variables used in the electron identification and in the kinematic requirements. 


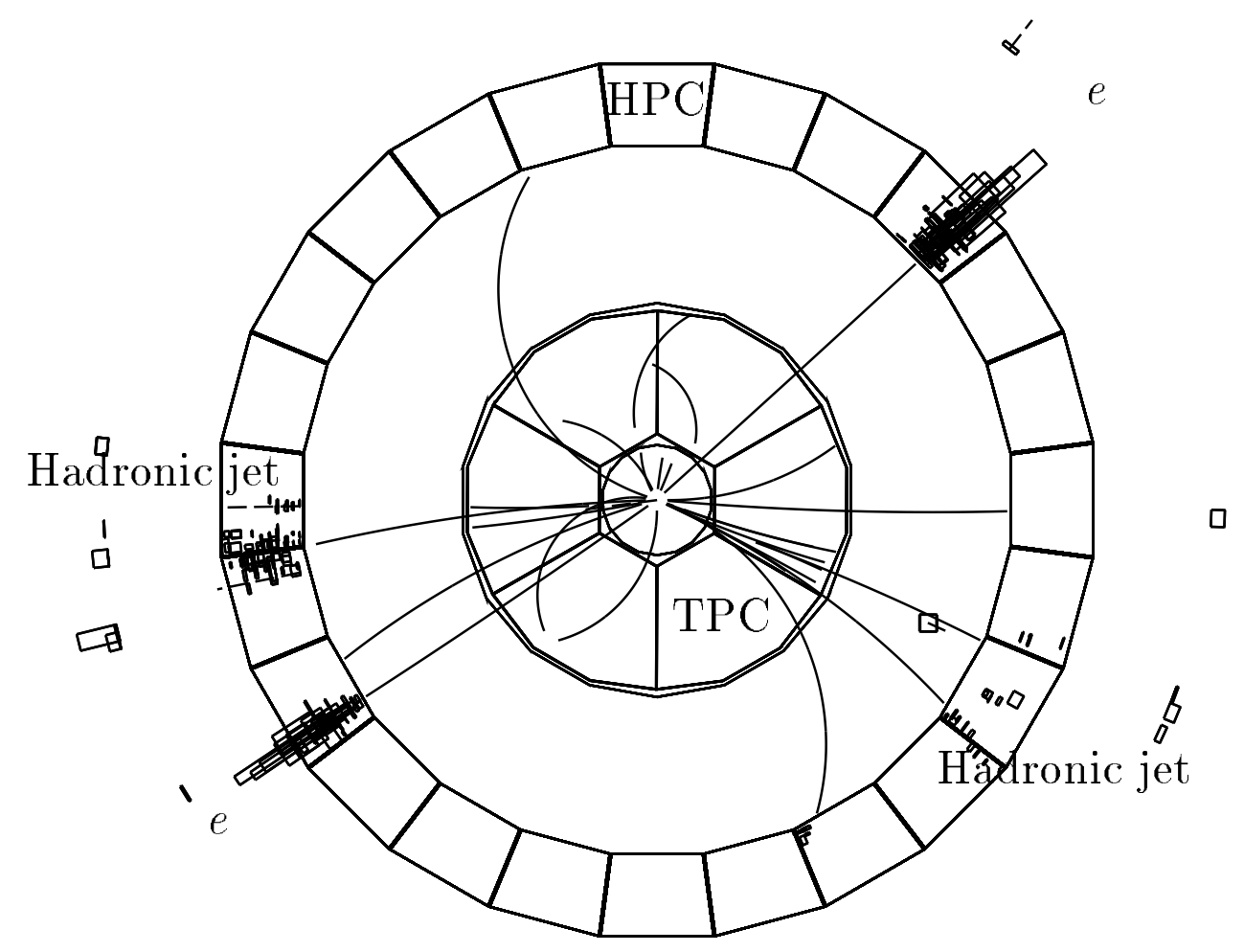

Figure 8: $\mathrm{He}^{+} \mathrm{e}^{-}$channel: an $\mathrm{R} \phi$ graphical representation of the event selected. The largest energy deposits seen in the barrel electromagnetic calorimeter (HPC) are due to the electrons.

In a similar way the systematic errors on the expected background have been estimated. A detailed comparison of PYTHIA to four-fermion generators for final states of the type $\mathrm{e}^{+} \mathrm{e}^{-} \mathrm{q} \overline{\mathrm{q}}$ has shown significant discrepancies that have been attributed to the less complete nature of the process described by PYTHIA. On the other hand no significant discrepancy, outside the statistical accuracy, has been observed between different four-fermion generators (EXCALIBUR, GRC4F and WPHACT). For this reason our background estimates for four-fermion final states are based on samples from four-fermion generators. The absence of FSR in EXCALIBUR, referred to in the $\mathrm{H} \mu^{+} \mu^{-}$search, is of less relevance in the electron channel, where it is minor in comparison to bremsstrahlung in the detector material.

One event fulfils the selection criteria. The electron energies are 19.6 and $55.6 \mathrm{GeV}$ and the maximum and minimum jet isolation angles are $82.7^{\circ}$ and $32.2^{\circ}$. The $4-\mathrm{C}$ fit gives an $\mathrm{e}^{+} \mathrm{e}^{-}$mass of $68.5 \mathrm{GeV} / c^{2}$ and a recoil mass of $87.5 \mathrm{GeV} / c^{2}$ with a fit probability of $4.5 \%$. When the 5 - $\mathrm{C}$ fit is performed, these numbers become $70.0 \mathrm{GeV} / c^{2}, 86.5 \mathrm{GeV} / c^{2}$ and $0.8 \%$ respectively. This event is depicted in Fig. 8.

\section{Neutral Higgs boson searches in events with jets and missing energy}

This topology corresponds to $20 \%$ of the $\mathrm{ZH}$ final states. The experimental signature is a pair of acollinear jets, acoplanar with the beam, coming mostly from b quarks, with a recoiling mass compatible with expectation from $\mathrm{Z} \rightarrow \nu \bar{\nu}$ decays.

A sample of hadronic events was defined by the following two criteria: 
- at least nine charged particles were required, and at least one of them had to originate from the reconstructed event primary vertex with an $R \phi$ impact parameter less than $200 \mu \mathrm{m}$;

- the total energy of detected charged particles had to exceed $0.1 \cdot \sqrt{s}$.

A veto based on hermeticity counters was applied to these events to reject events with an on-shell $\mathrm{Z}$ and photons at angles with poor electromagnetic calorimetry coverage. The total cross-section for $\mathrm{b} \bar{b} \gamma(\gamma)$ events with an energetic radiated photon in the detector acceptance is about $7 \mathrm{pb}$. Without the veto, these would give a background of $0.47 \pm 0.06$ events with partially reconstructed energetic photons in the candidate sample. The veto algorithm considers signals from hermeticity counters installed at polar angles of $40^{\circ}$ and $90^{\circ}$. Events with such signals were rejected if topologically and kinematically consistent with the hypothesis that jets accompanied by one photon in the direction of the counter give a signal and another photon (possibly of very low energy) is lost in the beam direction. The background due to poorly reconstructed photons is suppressed down to the level of $2 \mathrm{fb}$, i.e. $0.12 \pm 0.03$ expected events in the final selection, with a loss of $8 \%$ (relative) in efficiency.

\subsection{Iterative discriminant analysis}

Eleven variables were used to exploit the differences between the Higgs signal and the various background processes.

- $E_{\gamma} / E_{\gamma}^{Z}$ : the normalized energy of a photon, assumed to have escaped in the beam direction. It was computed from the polar angles of the two main jet directions in the event. The two jet directions were computed by forcing the event into two jets with the LUCLUS routine [21]. The estimated photon energy was normalized to the expected energy for a photon recoiling against an on-shell $\mathrm{Z}$.

- $\left|\cos \theta_{P}\right|:$ the absolute value of the cosine of the polar angle of the missing momentum.

- $E_{f} / E_{t o t}$ : the fraction of the total reconstructed energy below a polar angle of $20^{\circ}$.

- $E_{\text {cone }} / p_{\text {iso }}$ : the energy sum in a double cone, around the most isolated particle, divided by its momentum. The lower half opening angle was $5^{\circ}$ and the upper one is $\alpha_{\max }$. In the momentum interval $2-5 \mathrm{GeV} / c, \alpha_{\max }$ was set to $60^{\circ}$ in order to maximise the sensitivity to isolated particles from $\mathrm{W}^{+} \mathrm{W}^{-} \rightarrow q q \tau \nu$ events, while $\alpha_{\max }=25^{\circ}$ was used for higher momenta. The most isolated particle was defined as the particle with momentum above $2 \mathrm{GeV} / c$ with the smallest $E_{\text {cone }} / p_{\text {iso }}$.

- The momentum of the most isolated particle, as defined above.

- $E_{\text {tot }}$ : the total reconstructed energy of the event.

- $M_{v i s}$ : the reconstructed invariant mass of the event.

- $\log \left[\Delta \phi \cdot \sin \theta_{j e t}^{m i n}\right]$ : the logarithm to base ten of the scaled acoplanarity. Acoplanarity was defined as $\Delta \phi$, the complement of the difference in azimuthal angle between the two jets in degrees, when forcing the reconstruction to have exactly two jets. In order to compensate for the geometrical instability of this variable for jets at low polar angles, it was multiplied by the sine of the smaller polar angle of the two jets.

- The sum of the two smallest differences in $\cos \theta$ between a jet and a region of weaker energy resolution $\left(40^{\circ}\right.$ and $90^{\circ}$ polar angles).

- The thrust computed in the rest frame of the visible system. The transformation into the rest frame was made in order to compensate the smearing due to acollinearity of the jet system. 
- $x_{b}$ : the combined event b-tagging defined in Sect. 4.2 .

The invariant mass of the visible system given by a 1-C fit where the recoil system is an on shell $\mathrm{Z}$ boson, $M_{v i s}^{1 C}$, was also computed for selected events. The first column of Fig. 9 shows the distributions of $\left|\cos \theta_{P}\right|, \log \left[\Delta \phi \cdot \sin \theta_{\text {jet }}^{\text {min }}\right], M_{v i s}$, and $x_{b}$ following the hadronic preselection for real data, simulated background, and simulated signal.

Events which fell in the extreme tails of the selection variable distributions were removed. These 'tail cut' requirements (see Table 7) emphasize the signal region of the variable space in the discriminant analysis and avoid the effect of any badly reconstructed events with extreme variable values.

\begin{tabular}{lcc}
\hline Variable & Lower edge & Upper edge \\
\hline$\left|\cos \theta_{P}\right|$ & - & 0.95 \\
$E_{f} / E_{\text {tot }}$ & - & 0.20 \\
$E_{\text {tot }} / \sqrt{s}$ & 0.25 & 0.51 \\
$M_{v i s}$ & 31.0 & 107 \\
$\log \left[\Delta \phi \cdot \sin \theta_{j e t}^{\text {min }}\right]$ & -0.55 & - \\
$x_{b}$ & -2.42 & - \\
\hline
\end{tabular}

Table 7: $\mathrm{H} \nu \bar{\nu}$ channel: requirements made to remove events in tails of signal distributions.

The right column of Fig. 9 shows the distributions of four event variables following the 'tail cut' selection for real data, simulated background, and simulated signal. The overall agreement in the number of events for real and simulated data was within about $15 \%$ at this stage, with 239 observed events and 214 expected.

The remaining selection cuts are made with an Iterative Discriminant Analysis (IDA) $[22,23]$. A step in an IDA consists of defining a polynomial of event variables, such that the separation between signal and background is maximised [24]. The analysis used here consisted of two such steps, using quadratic discriminant functions on all the variables introduced in this section. After the first step the value of the discriminant function was required to exceed a value such that the efficiency is reduced by $30 \%$ on average for the signal samples. This left a much smaller sample where the background is suppressed relative to a possible signal. A second discriminant function was optimized for this sample. The distribution of its value for events passing the first IDA step is shown in Fig. 10 for data, simulated background and signal.

The background versus efficiency curve obtained by varying the requirement on the second discriminant step can be seen in Fig. 11 for a Higgs mass of $85 \mathrm{GeV} / c^{2}$. The final selection in the second IDA output has been chosen by the global optimization procedure (Sect. 4.5) and corresponds to a minimal value of 0.265. Table 8 and Fig. 29 show the efficiency, and Table 9 shows the number of events expected and observed at different stages in the analysis. Fig. 12 shows the selected candidate event. It has a visible mass of $77 \mathrm{GeV} / c^{2}$, which becomes $88 \mathrm{GeV} / c^{2}$ after the constrained fit, and $x_{b}$ is $1.2 .\left|\cos \theta_{P}\right|$ is 0.42 , and $\log \left[\Delta \phi \cdot \sin \theta_{j e t}^{m i n}\right]$ is 0.82 . The output weight from the second IDA is 0.37 .

At the end of the analysis, the Higgs boson mass is reconstructed as the mass of the visible system, found using a 1-C fit with the constraints of energy and momentum conservation and the constraint that the invisible system is an on-shell $\mathrm{Z}$. The distributions 

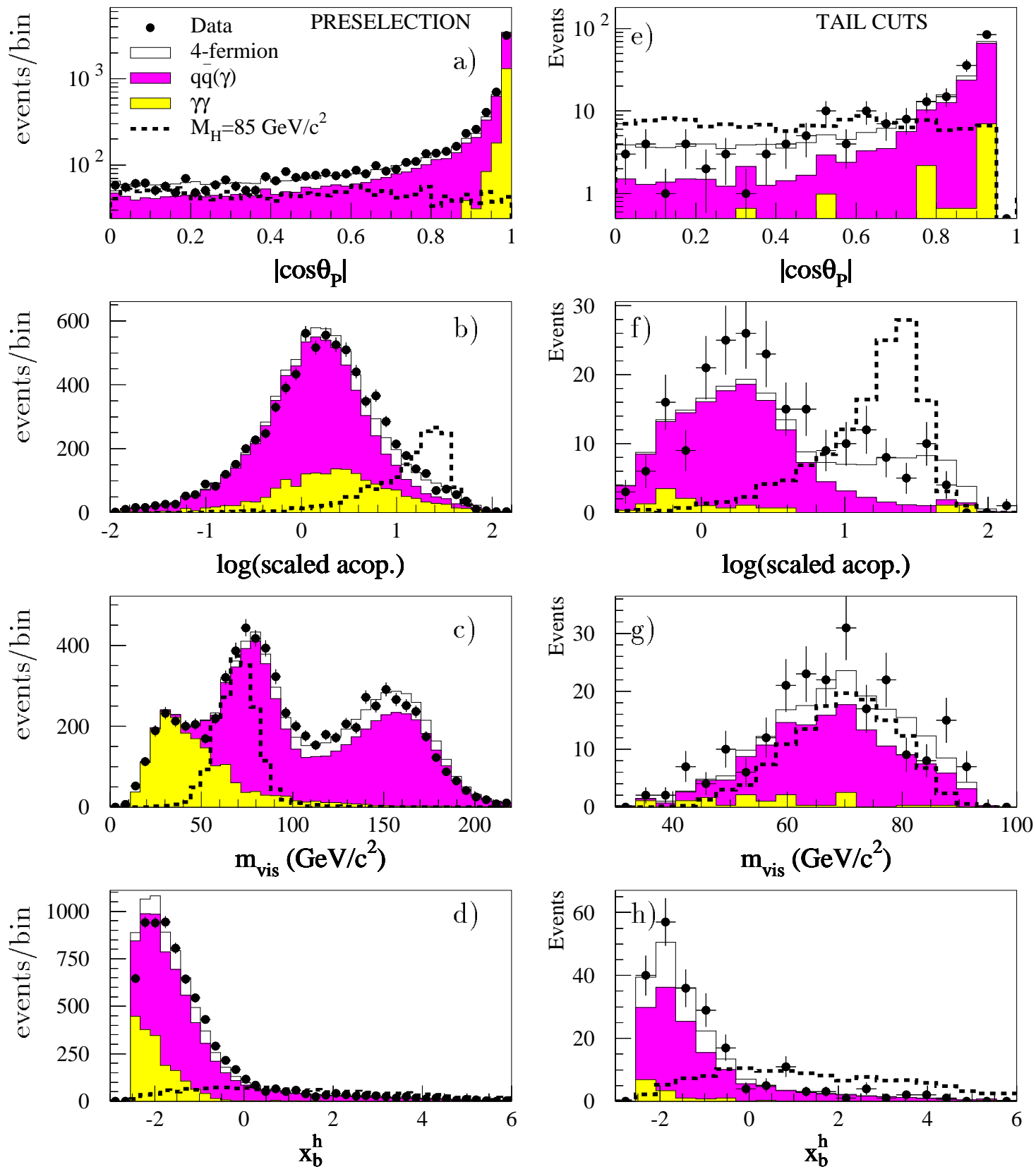

Figure 9: $\mathrm{H} \nu \bar{\nu}$ channel: a) to $\mathrm{d}$ ) show distributions of the four event variables used after hadronic preselection and e) to h) after 'tail cut' selection (see table 7). for real data (dots) and simulated background. The thick dashed histogram contour shows the expected signal for $m_{\mathrm{H}}=85 \mathrm{GeV} / c^{2}$ scaled up by factors of 500 at the preselection and 50 at the tail cuts stage. 


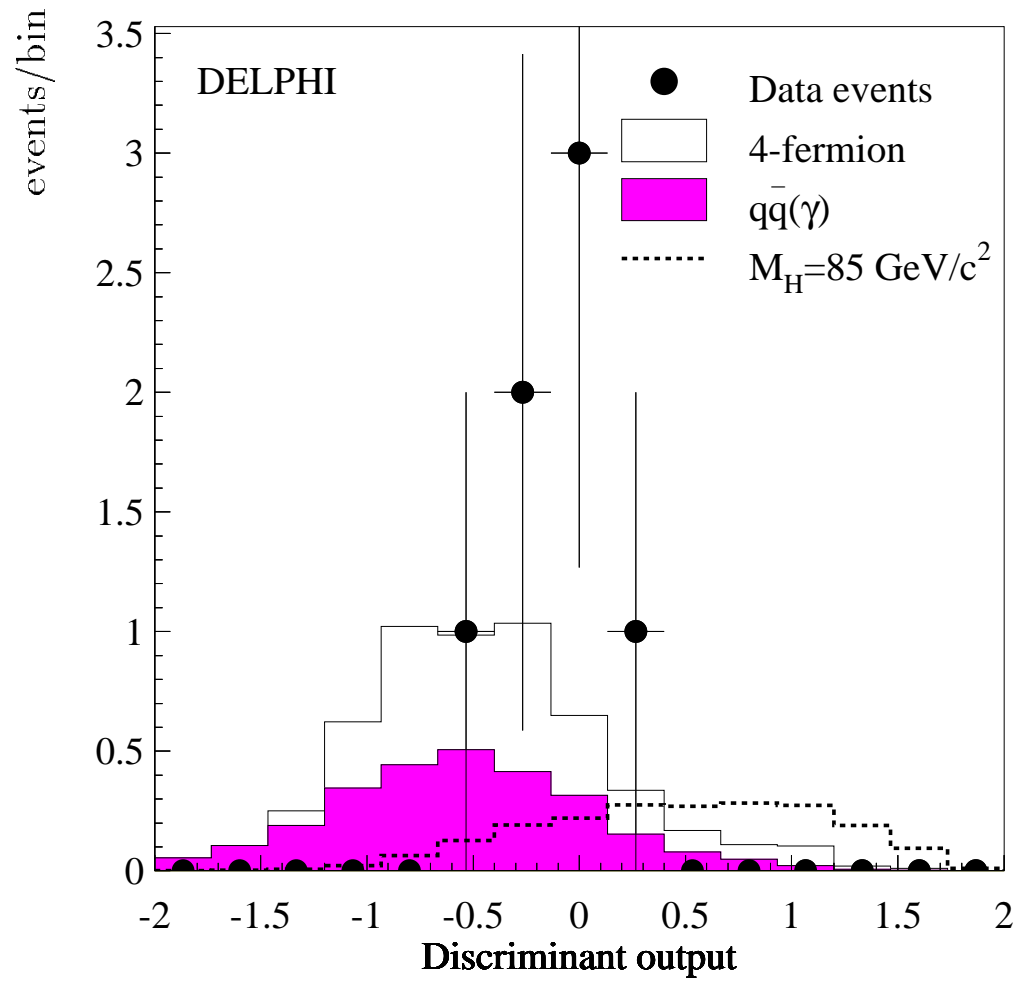

Figure 10: $\mathrm{H} \nu \bar{\nu}$ channel: distribution of the final discriminant output for those events which passed the first step in the Iterative Discriminant Analysis selection for data, background, and the expected signal for $m_{\mathrm{H}}=85 \mathrm{GeV} / c^{2}$. 


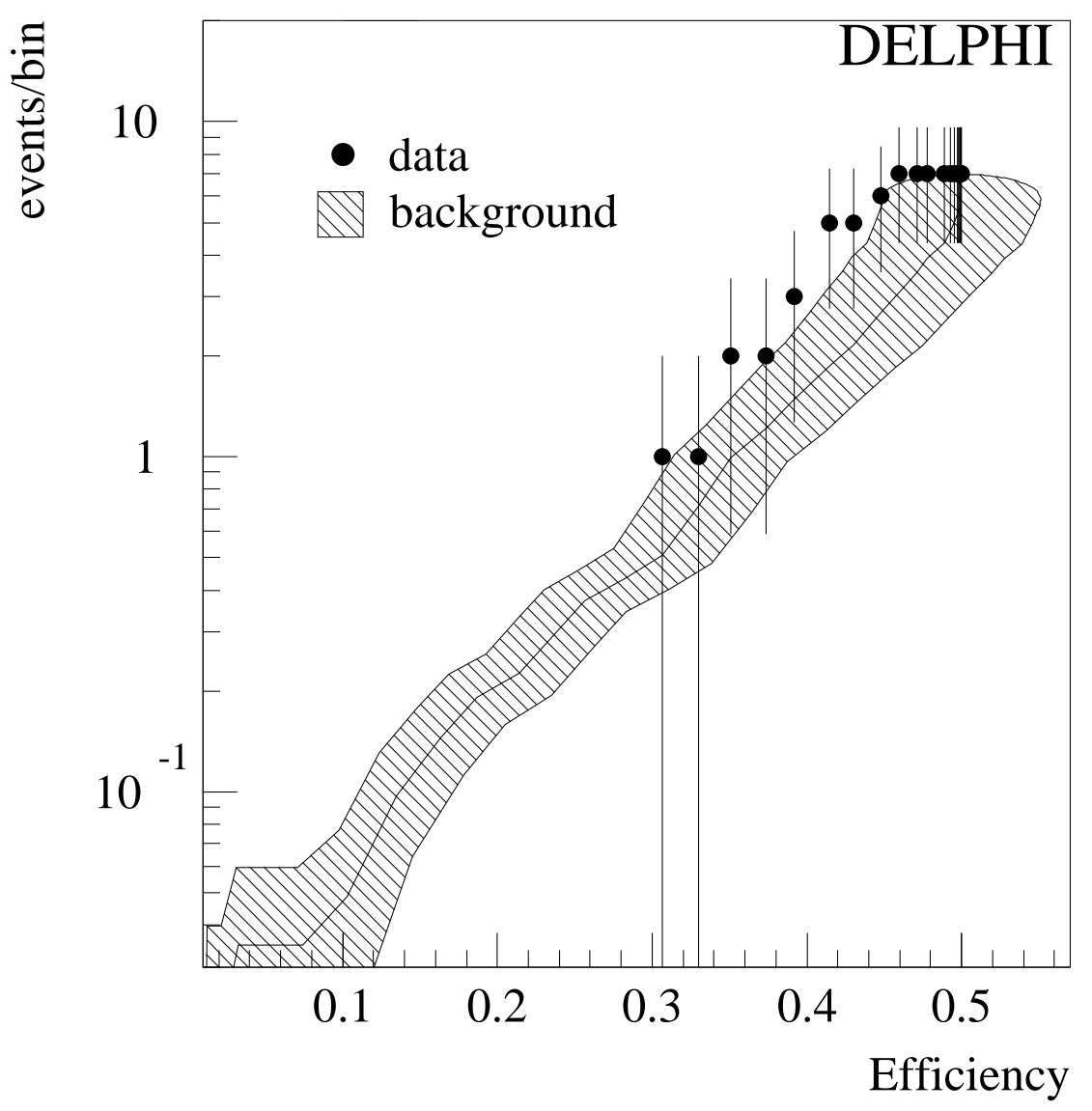

Figure 11: $\mathrm{H} \nu \bar{\nu}$ channel: background and number of observed events versus signal efficiency for different minimum requirements on the discriminant function. The shaded band shows one standard deviation uncertainties in signal efficiency and background, combined by adding them in quadrature. The efficiency cannot be greater than $50 \%$, as this is the fraction of events which pass the first Iterative Discriminant Analysis step. The uncertainties take into account simulation statistics and systematic effects. 

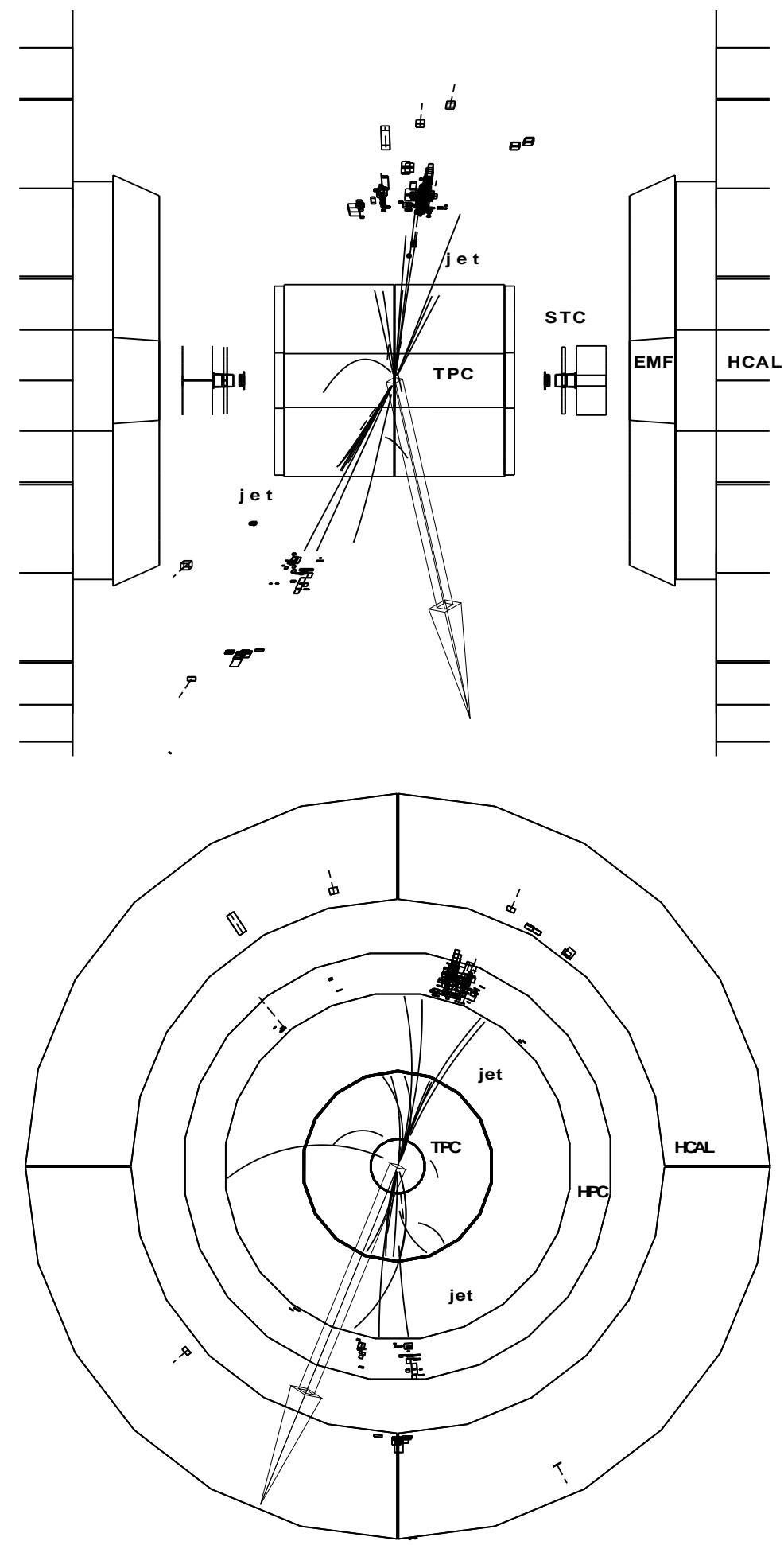

Figure 12: $\mathrm{H} \nu \bar{\nu}$ channel: longitudinal and transverse views of the selected candidate. The absence of signal in the forward detectors shows that there were no signals in these devices. The missing momentum in this event is indicated by the large arrow. 

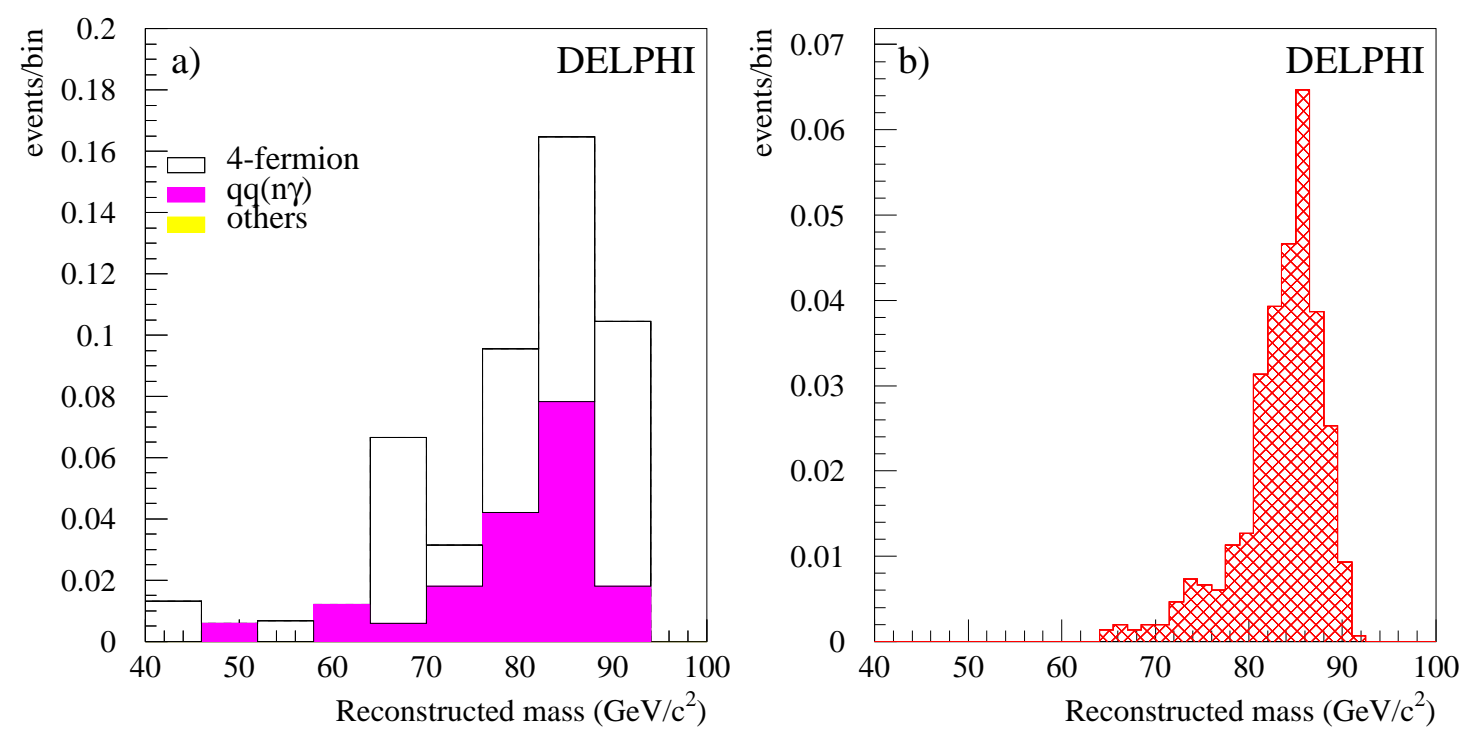

Figure 13: $\mathrm{H} \nu \bar{\nu}$ channel: distributions of the reconstructed Higgs boson mass in the final event selection for a) the expected background, and b) the signal $\left(m_{\mathrm{H}}=85 \mathrm{GeV} / c^{2}\right)$. The signal mass resolution is $2.9 \mathrm{GeV} / c^{2}$ (Gaussian fit). The one candidate has a mass of $88 \mathrm{GeV} / c^{2}$.

of the reconstructed Higgs boson mass expected for the signal and the background are shown in Fig. 13.

\subsection{Systematic uncertainties}

Estimates for signal efficiencies and background have been monitored by comparison of real and simulated data at all stages of the analysis (see Fig. 9 and Table 9). Several additional cross-checks, based on test samples, were performed in order to constrain the uncertainties in the final selection. Test samples were necessary to avoid biases since the statistics are low and the IDA might have been trained to recognise individual simulated signal events.

\begin{tabular}{cccc}
\hline$m_{\mathrm{H}}\left(\mathrm{GeV} / c^{2}\right)$ & Efficiency $(\%)$ & $m_{\mathrm{H}}\left(\mathrm{GeV} / c^{2}\right)$ & Efficiency $(\%)$ \\
\hline 60 & $40.6 \pm 1.5 \pm 2.4$ & 80 & $36.2 \pm 1.0 \pm 2.2$ \\
65 & $40.1 \pm 1.5 \pm 2.4$ & 85 & $31.6 \pm 1.2 \pm 1.9$ \\
70 & $39.9 \pm 1.0 \pm 2.4$ & 90 & $25.1 \pm 1.1 \pm 1.5$ \\
75 & $38.9 \pm 1.0 \pm 2.3$ & 95 & $21.1 \pm 1.2 \pm 1.3$ \\
\hline
\end{tabular}

Table 8: $\mathrm{H} \nu \bar{\nu}$ channel: the efficiency for various Higgs masses at the selected working point. The first uncertainties are due to Monte Carlo statistics, the second estimates give the systematic uncertainties. $85 \mathrm{GeV}$ have been checked 


\begin{tabular}{lccccccccc}
\hline & Data & $\begin{array}{c}\text { Total } \\
\text { background }\end{array}$ & $\mathrm{b} \overline{\mathrm{b}}(\gamma)$ & $\mathrm{q} \overline{\mathrm{q}}(\gamma)$ & $\mathrm{W}^{+} \mathrm{W}^{-}$ & $\mathrm{ZZ}$ & We $\nu$ & Others $\begin{array}{c}\mathrm{H} \nu \bar{\nu} \\
\varepsilon(\%)\end{array}$ \\
\hline Preselection & 7557 & 7978 & 1023 & 4003 & 689 & 47 & 19 & 2194 & 91 \\
Tail cuts & 218 & 201 & 34 & 101 & 35 & 3.1 & 9.5 & 19 & 66 \\
IDA, step 1 & 7 & 5.5 & 1.9 & 0.75 & 1.1 & 0.60 & 1.2 & 0 & 50 \\
IDA, step 2 & 1 & $0.50 \pm 0.08$ & 0.18 & 0.00 & 0.04 & 0.20 & 0.08 & 0 & 32 \\
\hline
\end{tabular}

Table 9: $\mathrm{H} \nu \bar{\nu}$ channel: the reduction for different background processes. The $\mathrm{b} \overline{\mathrm{b}}(\gamma)$ sample has been separated from the light quark $q \bar{q}(\gamma)$ sample. The column 'Others' includes two-photon processes, Zee and Bhabha scattering. The signal efficiency column is for $m_{\mathrm{H}}=85 \mathrm{GeV} / c^{2}$. Uncertainties include simulation statistics.

They were constructed from well-understood background processes and passed through the selection. Discrepancies between real and simulated data are expected to affect the test samples and the final sample in the normal selection in a similar way. The signal efficiencies were cross-checked using a test sample of events with hadronic systems recoiling against an isolated particle, such as $\mathrm{W}^{+} \mathrm{W}^{-} \rightarrow \mathrm{q} \overline{\mathrm{q}} \nu_{l}$ events and $\mathrm{q} \overline{\mathrm{q}}$ production with an isolated photon from initial state radiation. The isolated particle was used as a tag, and all the event variables were recomputed using the hadronic system only, which resembles a signal event. As an additional check, in order to increase the statistics late in the selection, the value of $x_{b}$ was systematically shifted to higher values. Good agreement between data and simulation was found for the rates and shapes of distributions including the b-tagging and event weights, as seen in Fig. 6.2 a) and b) and Table 10, first row.

\begin{tabular}{|c|c|c|c|c|c|}
\hline & Tail cuts & Step 1 & $\begin{array}{c}\text { Step } 1 \\
x_{b} \quad \text { shift }\end{array}$ & Final & $\begin{array}{c}\text { Final } \\
x_{b} \text { shift }\end{array}$ \\
\hline \multicolumn{6}{|c|}{ Test events, tagged by isolated particles } \\
\hline Data & 533 & 43 & 170 & 14 & 108 \\
\hline Simulation & $475 \pm 5$ & $42 \pm 1$ & $154 \pm 2$ & $16.3 \pm 0.5$ & $112 \pm 2$ \\
\hline \multicolumn{6}{|c|}{ Test events, rejected by the first discriminant analysis step } \\
\hline Data & 211 & 0 & 29 & 0 & 14 \\
\hline Simulation & $195 \pm 4$ & 0 & $28.8 \pm 1.3$ & 0 & $12.2 \pm 0.6$ \\
\hline \multicolumn{6}{|c|}{ Test events, tagged by a large STIC energy deposit } \\
\hline Data & 80 & 3 & & 0 & \\
\hline Simulation & $57 \pm 2$ & $3.1 \pm 0.5$ & & $0.93 \pm 0.5$ & \\
\hline
\end{tabular}

Table 10: $\mathrm{H} \nu \bar{\nu}$ channel: the numbers of observed and expected events in test samples consisting of hadronic systems recoiling against an isolated particle tag (upper part) of events rejected by the first discriminant iteration and $x_{b}$ artificially increased (middle part) and of hadronic systems recoiling against a photon tag in STIC (lower part). The columns give rates after the selection of tail cuts, the first iteration ('Step 1') and in the final sample ('Final'). 

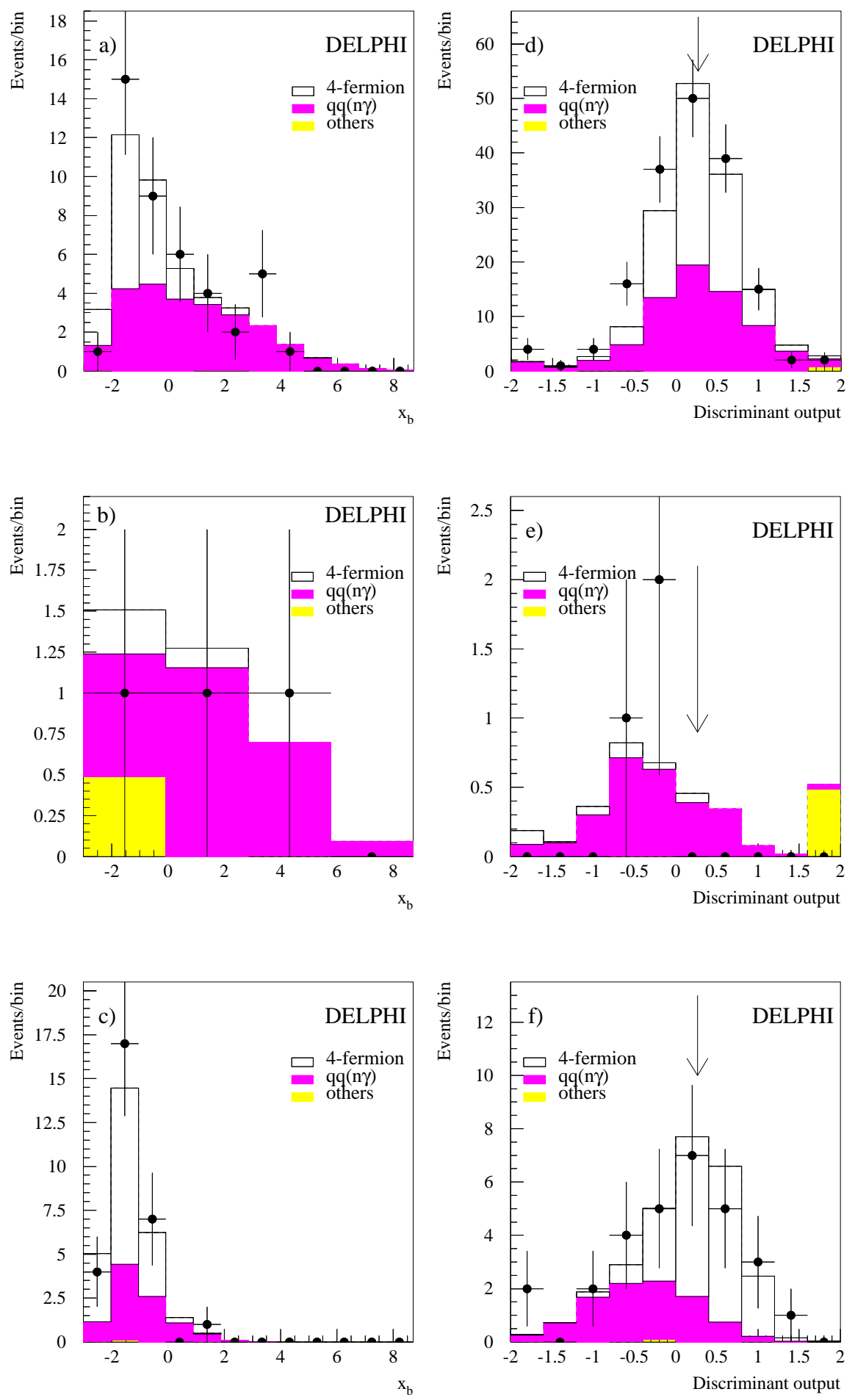

Figure 14: $\mathrm{H} \nu \bar{\nu}$ channel: distributions of beauty event tag in the test sample of hadronic systems recoiling against an isolated particle a), in the sample of hadronic systems recoiling against an isolated particle in the STIC acceptance b), and in the sample of events surviving the tail cuts but rejected after the first IDA step c). Distributions of the IDA weights for these test samples, are shown in d-f), respectively. The beauty weights are shifted up in computing the IDA function values in $d$ ) and $f$ ). The arrows show the position of the final selection. The observed and expected event rates are listed in Table 10 . 
The uncertainties in the signal efficiencies due to event reconstruction are limited to $\pm 10 \%$ relative, based on the last selection applied to the events tagged by isolated particles in Table 10, where 108 events were observed compared with 112 expected. In addition, the efficiency uncertainties due to fusion production graphs have been checked with the WPHACT [11] generator and found to be well within this range.

The background estimate in a missing energy search depends strongly on the modelling of rare occurrences in the detector, leading to lost or spurious particles. The precision in this modelling was evaluated using events which survive the tail-cut selection but were rejected in the first IDA step. Many of these events had a low b-tagging weight, $x_{b}$. By artifically increasing $x_{b}$, a more signal-like sample was obtained, dominated by $q \bar{q}(n \gamma)$, $\mathrm{W}^{+} \mathrm{W}^{-}$and $\mathrm{We} \nu$ events. These events had kinematic properties that were very close to the residual actual background. The observed and expected rates for this sample, shown in Table 10, second row, and their b-tagging and IDA weight distributions, shown in Fig. $6.2 \mathrm{c}$ )-d), are in good agreement. As a specific check of the important background from $\mathrm{b} \overline{\mathrm{b}}$ events with photons lost in the beam directions, high-energy deposits in the STIC were used to tag radiated photons which were then assumed lost in computing the event variables. Three events were observed after the first IDA step for an expected $3.1 \pm 0.2$ events (see Table 10, last row and Fig. $6.2 \mathrm{e}$ )-f)). The uncertainty of the background in the final selection is estimated to be $+-20 \%$, corresponding to the statistical uncertainty of the 29 events in the test sample.

\section{Neutral Higgs boson searches in events with jets and taus}

This topology includes $8.5 \%$ of the ZH final states and typically $14 \%$ of the hA final states. The experimental signature is two jets and two isolated $\tau$ leptons, which are reconstructed exclusively.

\subsection{Preselection}

Hadronic events are selected by requiring at least seven charged particles and, either a total energy carried by charged particles greater than $0.15 \sqrt{s}$, or a total energy greater than $0.3 \sqrt{s}$ and forward and backward energies greater than $0.03 \sqrt{s}$. After this hadronic selection, an exclusive search for $\tau$ leptons is applied. A cone algorithm is used to define clusters in the event. The two free parameters of the algorithm are the minimum energy in the cone $\left(E_{\min }\right)$ and the cone half opening angle $\left(\theta_{1 / 2}\right)$. Their values $\left(E_{\text {min }}=1.3 \mathrm{GeV}\right.$ and $\theta_{1 / 2}=0.2 \mathrm{rad}\left(11.5^{\circ}\right)$ have been determined by optimizing the signal over background ratio for this analysis.

The angles between all possible pairs of cones are computed using as possible $\tau$ candidates only cones with no other particles within $15^{\circ}$ of the cone axis. The principal $\tau$ decays are then tagged by means of several algorithms, depending on the charged and neutral multiplicities in the cones. The different algorithms are, by order of application, i.e. order of decreasing purity, as follows.

1. Lepton algorithm: lepton identification [13] is used together with an upper limit on the lepton momentum to reject decays of $\mathrm{W}$ or $\mathrm{Z}$ bosons to leptons.

2. $\rho$ algorithm: three different algorithms were designed, depending on the number of neutrals in the cone (one, two or more than two). Selection cuts are applied on the 
invariant mass and total momentum of the particles in the cone, as well as on the maximum opening angle between them.

3. $\pi^{ \pm}$algorithm: this is the most difficult channel due to the contamination from pions in hadronic jets. As these are softer than pions from $\tau$ decays, a minimum value is required for the pion momentum. A maximum value is also imposed to reject direct leptons from $\mathrm{W}$ decays not selected by the lepton algorithms.

4. Three prong algorithm: the three charged particles in the cone are required to form a secondary vertex within $1.9 \mathrm{~mm}$ of the primary vertex, to be isolated from the rest of the event and to be at small angles with respect to one another.

The one-prong algorithms (1 to 3 ) have a selection efficiency from $50 \%$ to $67 \%$ for one-prong $\tau$ decays, while the three-prong algorithm has an efficiency of $28 \%$. The contamination from fake $\tau$ candidates reconstructed from hadronic particles of a jet ranges from $5 \%$ in the $\mu$ channel, through $11 \%$ in the electron channel, $15 \%$ in the $\pi^{ \pm}$channel, $16-22 \%$ for the $\rho$ channel to $28 \%$ for the $3 \pi$ channel. More details can be found in [25].

Events with no $\tau$ candidate are rejected. Events with two or more $\tau$ candidates are kept for the analysis and make a sample that will be called $2 \tau$ in the following (approximately $30 \%$ of the signal). In the case of more than two candidates, the candidates selected by the less efficient and less pure algorithms are not considered further.

In order to increase the signal efficiency, events with only one $\tau$ candidate are kept and a second $\tau$ is searched for with a looser selection than before: identified leptons with moderate momenta or well isolated cones failing the $\tau$ algorithm selection cuts are again considered and kept if they are of opposite charge to the first $\tau$ candidate and isolated from it. If no second $\tau$ is found, the missing momentum direction is used to define a preferred region in which an isolated track of moderate momentum with charge opposite to that of the first $\tau$ candidate is considered as a second $\tau$ candidate. This constitutes a sample called $(1+1) \tau$ in the following (approximately $50 \%$ of the signal).

In both samples, the preselection is completed by a requirement on the effective centreof-mass energy $\sqrt{s^{\prime}}[26]$ to reduce the background further. The effect of the preselection is shown in Table 11. At this level, the dominant backgrounds are $\mathrm{W}^{+} \mathrm{W}^{-}$and $q \bar{q}(\gamma)$ events. The agreement between data and background simulation is good.

\begin{tabular}{cccccccccc}
\hline Selection & Data & $\begin{array}{c}\text { Total } \\
\text { background }\end{array}$ & $\mathrm{q} \overline{\mathrm{q}}(\gamma)$ & $\mathrm{W}^{+} \mathrm{W}^{-}$ & ZZ & Zee & We $\nu$ & $\begin{array}{c}\gamma \gamma \\
\text { had. }\end{array}$ & $\begin{array}{c}\text { ZH } \\
\varepsilon(\%)\end{array}$ \\
\hline Hadronic & 7016 & $7522 \pm 17$ & 5142 & 742 & 55.0 & 118.9 & 20.3 & 1061 & 99.7 \\
\hline $2 \tau$ & 56 & $55.6 \pm 1.7$ & 20.1 & 16.6 & 2.8 & 3.4 & 0.1 & 12.4 & 32.0 \\
$\sqrt{s^{\prime}}>110$ & 27 & $29.1 \pm 1.1$ & 8.5 & 12.1 & 2.4 & 2.2 & 0.04 & 3.7 & 29.3 \\
\hline Selection & data & total & $\mathrm{q} \overline{\mathrm{q}}(\gamma)$ & $\mathrm{W}^{+} \mathrm{W}^{-}$ & ZZ & Zee & We $\nu$ & $\gamma \gamma$ & ZH (hA) \\
\multicolumn{7}{c}{ background } \\
\hline $1+1 \tau$ & 208 & $209.5 \pm 3.5$ & 103.4 & 52.7 & 6.3 & 7.9 & 0.3 & 38.5 & $48.1(49.9)$ \\
$\sqrt{s^{\prime}}>110$ & 120 & $118.8 \pm 2.4$ & 48.5 & 45.1 & 5.5 & 4.7 & 0.2 & 14.5 & $44.0(42.5)$ \\
\hline
\end{tabular}

Table 11: $\tau^{+} \tau^{-} \mathrm{q} \overline{\mathrm{q}}$ channel: effect of the preselection on data and simulated background at $\sqrt{s}=183 \mathrm{GeV}$. The cut on $\sqrt{s^{\prime}}$ is in $\mathrm{GeV}$. Efficiencies are given for signals at $85 \mathrm{GeV} / c^{2}$ in the $\mathrm{ZH}$ channel, and at $70 \mathrm{GeV} / c^{2}$ in the hA channel. 
Before channel-dependent selection cuts, all particles except those belonging to the two $\tau$ candidates are forced into two jets with the DURHAM algorithm [20]. Masses derived from application of a 4-C kinematic fit are used in the event selection, while the estimator of the Higgs boson mass used for the limit is the 5 - $\mathrm{C}$ fit value.

\subsection{The $\mathrm{ZH}$ channel}

\subsection{1 $\quad \mathbf{H}$ decaying into $\tau^{+} \tau^{-}$}

In the $\mathrm{ZH}$ channel with $\mathrm{H}$ decaying into $\tau^{+} \tau^{-}$, the $2 \tau$ sample is used to achieve a good purity, for lack of other powerful discriminating variables to reject four-fermion events, and especially WW pairs. The details of the selection cuts as well as their effect on data and simulated events are given in Table 12. The background is reduced by requiring the momentum of the less energetic jet, $P_{q 2}$, to be bounded, the mass of the $\tau$ pair, $M_{\tau \tau}$, to be high (since the search is restricted to high mass Higgs bosons) and the mass of the hadronic system, $M_{q \bar{q}}$, to be compatible with $m_{\mathrm{Z}}$. The final two selection cuts use the opening angle of the $\tau$ pair, expected to be large if the Higgs boson is massive, and the difference between the second and fourth Fox-Wolfram moments of the hadronic system, $H_{2}-H_{4}$, expected to be larger for the signal than for the background. Their values have been varied and the final choice determined in the global optimization procedure.

\begin{tabular}{ccccccccccc}
\hline selection & data & $\begin{array}{c}\text { total } \\
\text { background }\end{array}$ & $\mathrm{q} \overline{\mathrm{q}}(\gamma)$ & $\mathrm{W}^{+} \mathrm{W}^{-}$ & ZZ & Zee We $\nu$ & $\begin{array}{c}\gamma \gamma \\
\text { had. }\end{array}(\%)$ \\
\hline $7<P_{q 2}<50 \mathrm{GeV} / c$ & 18 & $20.0 \pm 0.9$ & 5.6 & 8.4 & 2.1 & 1.6 & 0.01 & 2.1 & 26.0 \\
$M_{\tau \tau}>60 \mathrm{GeV} / c^{2}$ & 3 & $6.35 \pm 0.35$ & 1.34 & 2.77 & 0.76 & 0.28 & 0.0 & 1.01 & 23.6 \\
$70<M_{q q}<110 \mathrm{GeV} / c^{2}$ & 1 & $1.47 \pm 0.14$ & 0.37 & 0.62 & 0.47 & 0.0 & 0.0 & 0.0 & 21.7 \\
\hline$\theta_{\tau \tau}>124.6^{\circ}$ & 1 & $1.00 \pm 0.11$ & 0.30 & 0.26 & 0.44 & 0.0 & 0.0 & 0.0 & 21.6 \\
$\left(H_{2}-H_{4}\right)>0.09$ & 1 & $0.74 \pm 0.09$ & 0.16 & 0.17 & 0.41 & 0.0 & 0.0 & 0.0 & 20.0 \\
\hline
\end{tabular}

Table 12: $\left(\mathrm{H} \rightarrow \tau^{+} \tau^{-}\right) q \bar{q}$ channel: effect of the final selection cuts in data and simulated background. Efficiencies are given for a signal of $85 \mathrm{GeV} / c^{2}$.

Table 13 and Fig. 29 show the signal efficiencies for different Higgs boson masses. The agreement between data and background simulation is illustrated in Fig. 15 at preselection level.

\begin{tabular}{cccc}
\hline$m_{\mathrm{H}}\left(\mathrm{GeV} / c^{2}\right)$ & Efficiency $(\%)$ & $m_{\mathrm{H}}\left(\mathrm{GeV} / c^{2}\right)$ & Efficiency $(\%)$ \\
\hline 60 & $1.6 \pm 0.3 \pm 0.1$ & 80 & $20.6 \pm 0.6 \pm 1.6$ \\
65 & $4.3 \pm 0.4 \pm 0.3$ & 85 & $20.0 \pm 0.6 \pm 1.6$ \\
70 & $8.3 \pm 0.6 \pm 0.6$ & 90 & $20.3 \pm 0.6 \pm 1.6$ \\
75 & $16.0 \pm 0.6 \pm 1.2$ & 95 & $17.1 \pm 0.8 \pm 1.3$ \\
\hline
\end{tabular}

Table 13: $\left(\mathrm{H} \rightarrow \tau^{+} \tau^{-}\right) q \bar{q}$ channel: efficiency of the Higgs boson selection at $\sqrt{s}=183 \mathrm{GeV}$ as a function of the Higgs boson mass. The first uncertainty quoted is statistical, the second is systematic. 

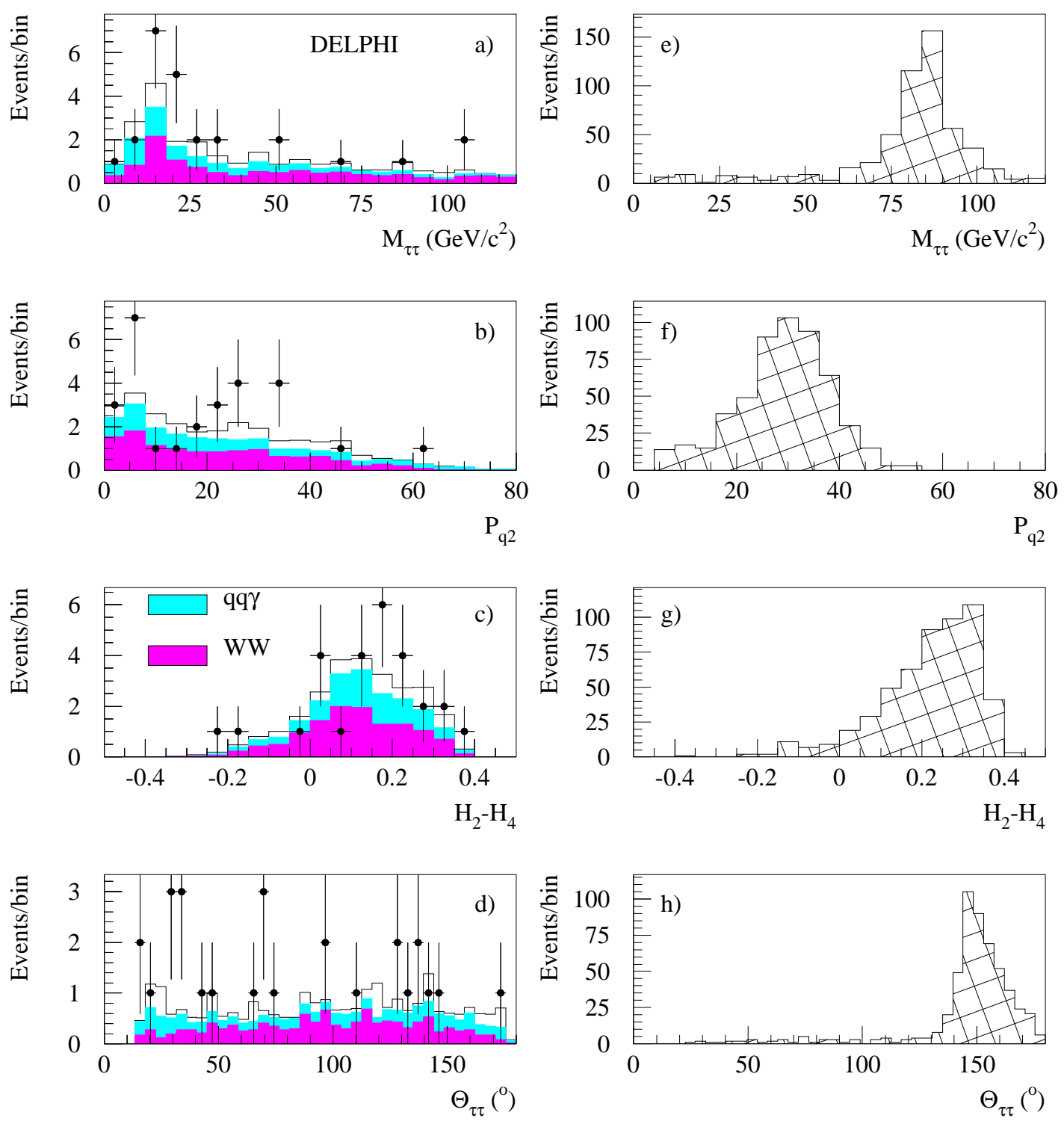

Figure 15: $\left(\mathrm{H} \rightarrow \tau^{+} \tau^{-}\right) q \bar{q}$ channel: plots a) to d) compare data (dots) with expected background distributions (full line) at $\sqrt{s}=183 \mathrm{GeV}$. The clear histogram is all other backgrounds. In e) to h), the unnormalized distributions expected for a Higgs signal at $85 \mathrm{GeV} / c^{2}$ are given. 


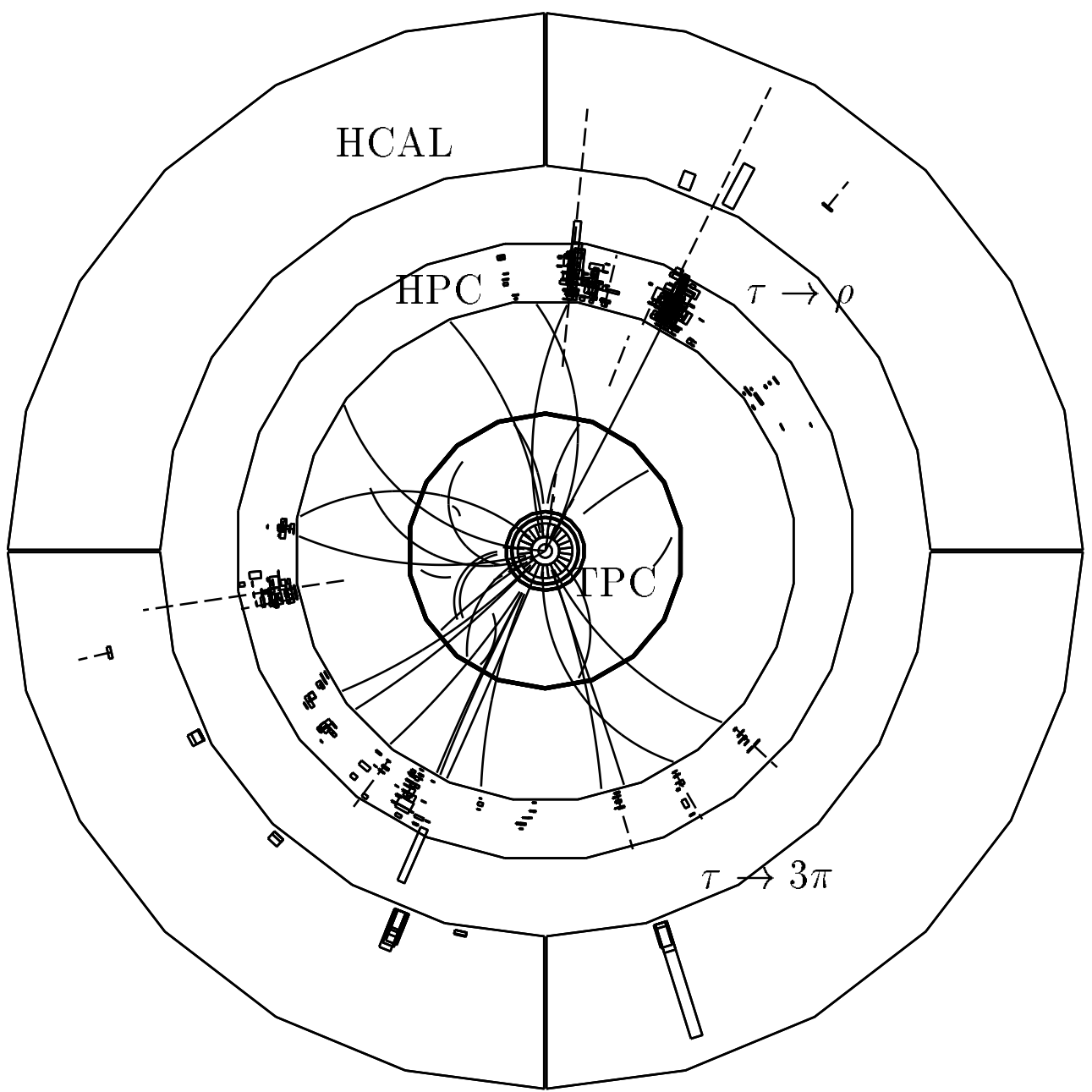

Figure 16: $\left(\mathrm{H} \rightarrow \tau^{+} \tau^{-}\right) \mathrm{q} \overline{\mathrm{q}}$ channel: $\mathrm{ZH}$ candidate. One $\tau$ candidate $(\tau \rightarrow \rho)$ goes to the upper right, where a high momentum charged particle can be seen to coincide with a large energy deposit in the electromagnetic calorimeter (HPC). The other $(\tau \rightarrow 3 \pi)$ can be seen in the lower right part of the figure. Three of the charged particles all have a common initial direction. The missing neutrinos are allowed for in the analysis.

The expected background is $0.74 \pm 0.09 \pm 0.08$ mainly from $\mathrm{ZZ}$ events. The one event selected in the data is shown in Fig. 16. The $\tau$ candidates are selected by the three prong channel and by the $\rho$ algorithm with at least three neutrals. The event corresponds to a reconstructed Higgs boson mass of $72 \mathrm{GeV} / c^{2}$ after the 5 -C fit.

\subsubsection{Z decaying into $\tau^{+} \tau^{-}$}

When the $\mathrm{Z}$ decays into a $\tau^{+} \tau^{-}$pair, the hadronic system is expected to come from the Higgs boson and thus to contain beauty hadrons. B-tagging is expected to be a powerful tool against background and the full preselected $(1+1) \tau$ sample is used in this analysis. Background reduction is achieved by requiring the mass of the hadronic system to be large and that of the $\tau$ pair to be compatible with $m_{\mathrm{Z}}$. The final two selection cuts apply on the opening angle of the $\tau$ pair and the event combined b-tagging variable. Their values have been varied and the final choice determined by the global optimization procedure. The result is indicated in Table 14, while Table 15 and Fig. 29 give the selection efficiency 
as a function of the Higgs boson mass. The four-fermion generator has been discussed in Sect. 2 .

\begin{tabular}{ccccccc}
\hline Selection & Data & $\begin{array}{c}\text { Total } \\
\text { Background }\end{array}$ & & & & \\
& \multicolumn{7}{c}{$(\gamma)$} & 4 fermions & $\begin{array}{c}\gamma \gamma \\
\text { had. }\end{array}$ & $\begin{array}{c}\text { ZH } \\
(\%)\end{array}$ \\
\hline$M_{q q}>60 \mathrm{GeV} / c^{2}$ & 84 & $81.0 \pm 1.6$ & 34.9 & 44.98 & 2.5 & 42.3 \\
$75<M_{\tau \tau}<105 \mathrm{GeV} / c^{2}$ & 7 & $8.44 \pm 0.64$ & 2.58 & 4.72 & 0.71 & 30.7 \\
\hline$\theta_{\tau \tau}>135.0$ & 3 & $5.80 \pm 0.59$ & 2.16 & 3.12 & 0.0 & 29.5 \\
$x_{\mathrm{b}}^{i}>-0.68$ & 0 & $0.34 \pm 0.07$ & 0.16 & 0.18 & 0.0 & 20.0 \\
\hline
\end{tabular}

Table 14: $(\mathrm{H} \rightarrow \mathrm{q} \overline{\mathrm{q}}) \tau^{+} \tau^{-}$channel: effect of the final selection cuts in real and simulated data at $\sqrt{s}=183 \mathrm{GeV}$. Efficiencies are given for a signal of $85 \mathrm{GeV} / c^{2}$.

\begin{tabular}{cccc}
\hline$m_{\mathrm{H}}\left(\mathrm{GeV} / c^{2}\right)$ & Efficiency $(\%)$ & $m_{\mathrm{H}}\left(\mathrm{GeV} / c^{2}\right)$ & Efficiency $(\%)$ \\
\hline 60 & $2.3 \pm 0.3 \pm 0.2$ & 80 & $19.7 \pm 0.6 \pm 1.5$ \\
65 & $7.5 \pm 0.6 \pm 0.6$ & 85 & $20.0 \pm 0.7 \pm 1.6$ \\
70 & $9.6 \pm 0.7 \pm 0.7$ & 90 & $19.5 \pm 0.6 \pm 1.5$ \\
75 & $15.9 \pm 0.6 \pm 1.2$ & 95 & $16.0 \pm 0.8 \pm 1.2$ \\
\hline
\end{tabular}

Table 15: $(\mathrm{H} \rightarrow \mathrm{q} \overline{\mathrm{q}}) \tau^{+} \tau^{-}$channel: efficiency of the Higgs boson selection $\sqrt{s}=183 \mathrm{GeV}$, as a function of the Higgs boson mass. The first uncertainty quoted is statistical, the second is systematic.

The data and simulated background after the preselection are compared in Fig. 17. Distributions of two variables used in the channel-dependent analysis are shown. There is no selected event in the data for an expected background of $0.34 \pm 0.07$ (stat.) \pm 0.04 (sys.) events, mainly due to $q \bar{q}(\gamma)$ events.

\subsection{The hA channel}

In the hA channel, the cross-section is typically maximum at large $\tan \beta$, i.e. when the two Higgs bosons are almost degenerate in mass. In that case, the masses of the pair of hadronic jets and of the $\tau^{+} \tau^{-}$pair are expected to be close. In addition, one Higgs boson is expected to decay into a b $\bar{b}$ pair. The starting sample is thus the entire $(1+1) \tau$ sample.

Background reduction is achieved through selection cuts in the acollinearity between the two hadronic jets, Acol had. $_{\text {. }}$ in the first Fox-Wolfram moment of the hadronic system, $H_{1}$, in the mass of the $\tau$ pair and the difference in mass between the $\tau$ pair and the hadronic system, $\Delta M$, and in the combined event b-tagging. The effect of the selection cuts on real and simulated data are given in Table 16. The last three requirements in the table have been determined through the global optimization procedure. The selection efficiencies as functions of the Higgs boson mass are given in Table 17 for two values of $\tan \beta$. 

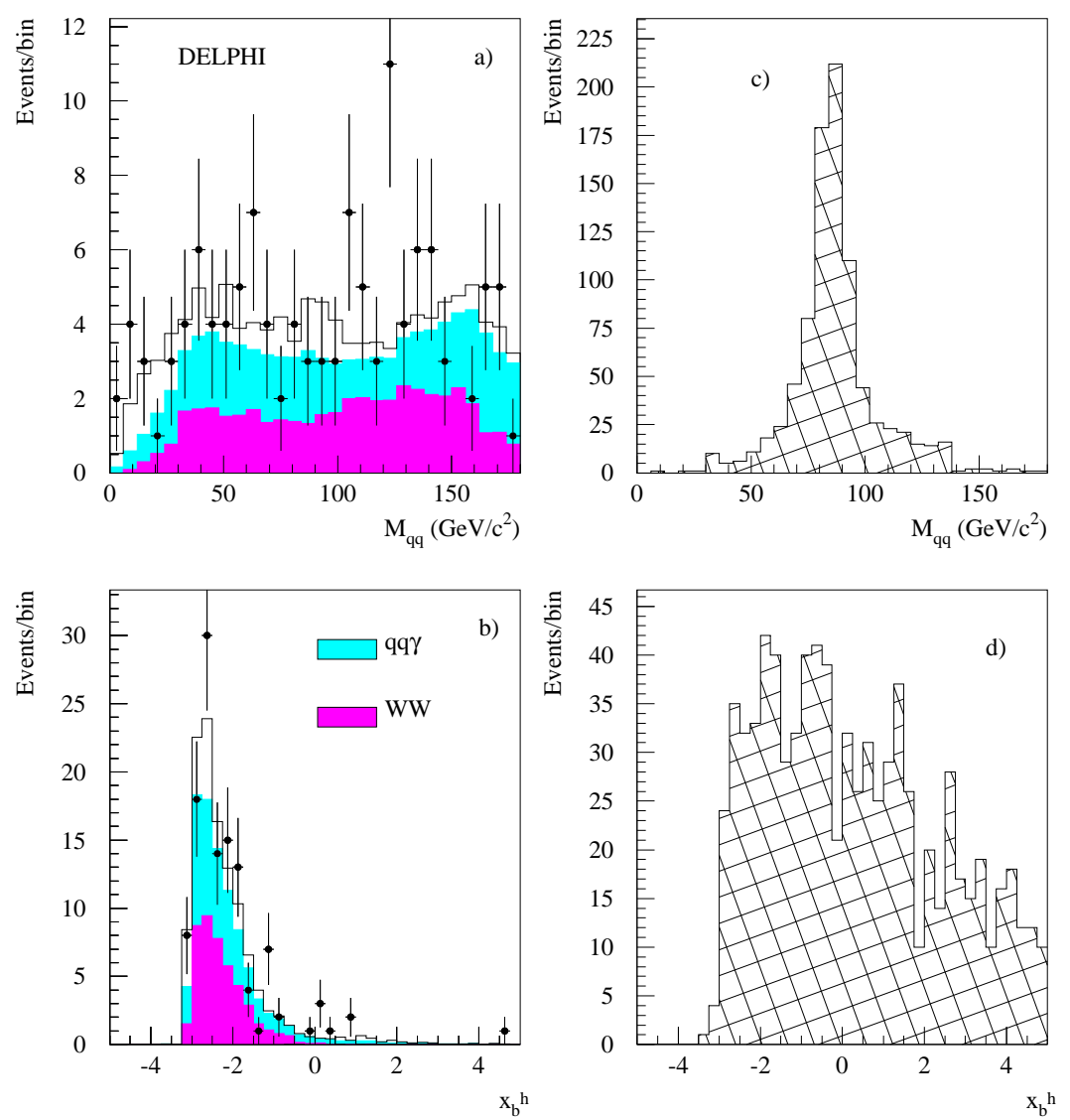

Figure 17: $(\mathrm{H} \rightarrow \mathrm{q} \overline{\mathrm{q}}) \tau^{+} \tau^{-}$channel: data (dots) are compared with expected background distributions (full line) in plots a) and b); the clear histogram is all other backgrounds. Plots c) and d) give unnormalized distributions expected for a Higgs signal at $85 \mathrm{GeV} / \mathrm{c}^{2}$. 


\begin{tabular}{cccccccccc}
\hline Selection & Data & $\begin{array}{c}\text { Total } \\
\text { background }\end{array}$ & $\mathrm{q} \overline{\mathrm{q}}(\gamma)$ & $\mathrm{W}^{+} \mathrm{W}^{-}$ & ZZ & Zee We $\nu$ & $\begin{array}{c}\gamma \gamma \\
\rightarrow \text { had. }\end{array}$ & $\begin{array}{c}\mathrm{hA} \\
(\%)\end{array}$ \\
\hline $0.7<$ Acol $_{\text {had. }}<2.0$ & 41 & $37.4 \pm 1.2$ & 13.1 & 18.3 & 1.9 & 1.7 & 0.07 & 2.3 & 35.5 \\
$H_{1}<0.7$ & 34 & $31.5 \pm 1.1$ & 10.3 & 15.2 & 1.8 & 1.7 & 0.05 & 2.3 & 34.9 \\
$M_{\tau \tau}>50 \mathrm{GeV} / c^{2}$ & 18 & $20.1 \pm 1.0$ & 6.3 & 9.4 & 0.7 & 1.3 & 0.04 & 2.3 & 32.4 \\
\hline$H_{1}>0.255$ & 11 & $12.58 \pm 0.73$ & 4.67 & 5.92 & 0.38 & 0.0 & 0.03 & 0.0 & 29.8 \\
$\Delta M<52 \mathrm{GeV} / c^{2}$ & 6 & $6.58 \pm 0.31$ & 2.86 & 3.38 & 0.31 & 0.0 & 0.02 & 0.0 & 29.1 \\
$x_{\mathrm{b}}^{i}>-0.85$ & 0 & $0.47 \pm 0.07$ & 0.31 & 0.10 & 0.04 & 0.0 & 0.0 & 0.0 & 22.6 \\
\hline
\end{tabular}

Table 16: hA $\rightarrow \tau^{+} \tau^{-} \mathrm{q} \overline{\mathrm{q}}$ channel: effect of the final selection cuts in real and simulated data at $\sqrt{s}=183 \mathrm{GeV}$. Efficiencies are given for a signal of $70 \mathrm{GeV} / c^{2}$ and $\tan \beta$ of 20 .

\begin{tabular}{ccc}
\hline$m_{\mathrm{A}}$ & \multicolumn{2}{c}{ Efficiency $(\%)$} \\
$\mathrm{GeV} / c^{2}$ & $(\tan \beta=2)$ & $(\tan \beta=20)$ \\
\hline 55 & $6.3 \pm 0.5 \pm 0.5$ & $10.9 \pm 0.7 \pm 0.9$ \\
60 & $11.5 \pm 0.7 \pm 0.9$ & $16.4 \pm 0.8 \pm 1.3$ \\
65 & $16.5 \pm 0.8 \pm 1.3$ & $22.0 \pm 0.9 \pm 1.7$ \\
70 & $20.6 \pm 0.6 \pm 1.6$ & $22.6 \pm 0.7 \pm 1.8$ \\
75 & $20.7 \pm 0.9 \pm 1.6$ & $21.6 \pm 0.9 \pm 1.7$ \\
\hline
\end{tabular}

Table 17: hA $\rightarrow \tau^{+} \tau^{-} \mathrm{q} \overline{\mathrm{q}}$ channel: efficiency of the Higgs boson selection at $\sqrt{s}=183 \mathrm{GeV}$, as a function of the common Higgs boson mass $m_{\mathrm{A}}$. The first uncertainty quoted is statistical, the second is systematic.

Figure 18 shows good agreement between data and simulated background for three analysis variables at the preselection level. At the end of the analysis, no event is selected in the data for an expected background of $0.47 \pm 0.07$ (stat.) \pm 0.05 (sys.), mainly due to $q \bar{q}(\gamma)$ events.

\subsection{Systematic errors}

A $1 \%$ systematic error is assumed on the background processes cross-sections. For each variable, the difference in the mean values of the distributions in data and simulated background is applied as a shift in the selection and the corresponding change in signal efficiency and background expectation is taken as the systematic uncertainty. The total error due to this is $\pm 6 \%$. The signal samples are generated without $\tau$ polarization. A sample with one Higgs mass was generated with $\tau$ polarization included, and the relative change in efficiency of $5 \%$ is taken as the error due to ignoring this effect.

All uncertainties are then summed quadratically. This leads to a $\pm 10 \%$ relative systematic error on the background expectations, and a $\pm 7.2 \%$ relative error on signal efficiencies. 

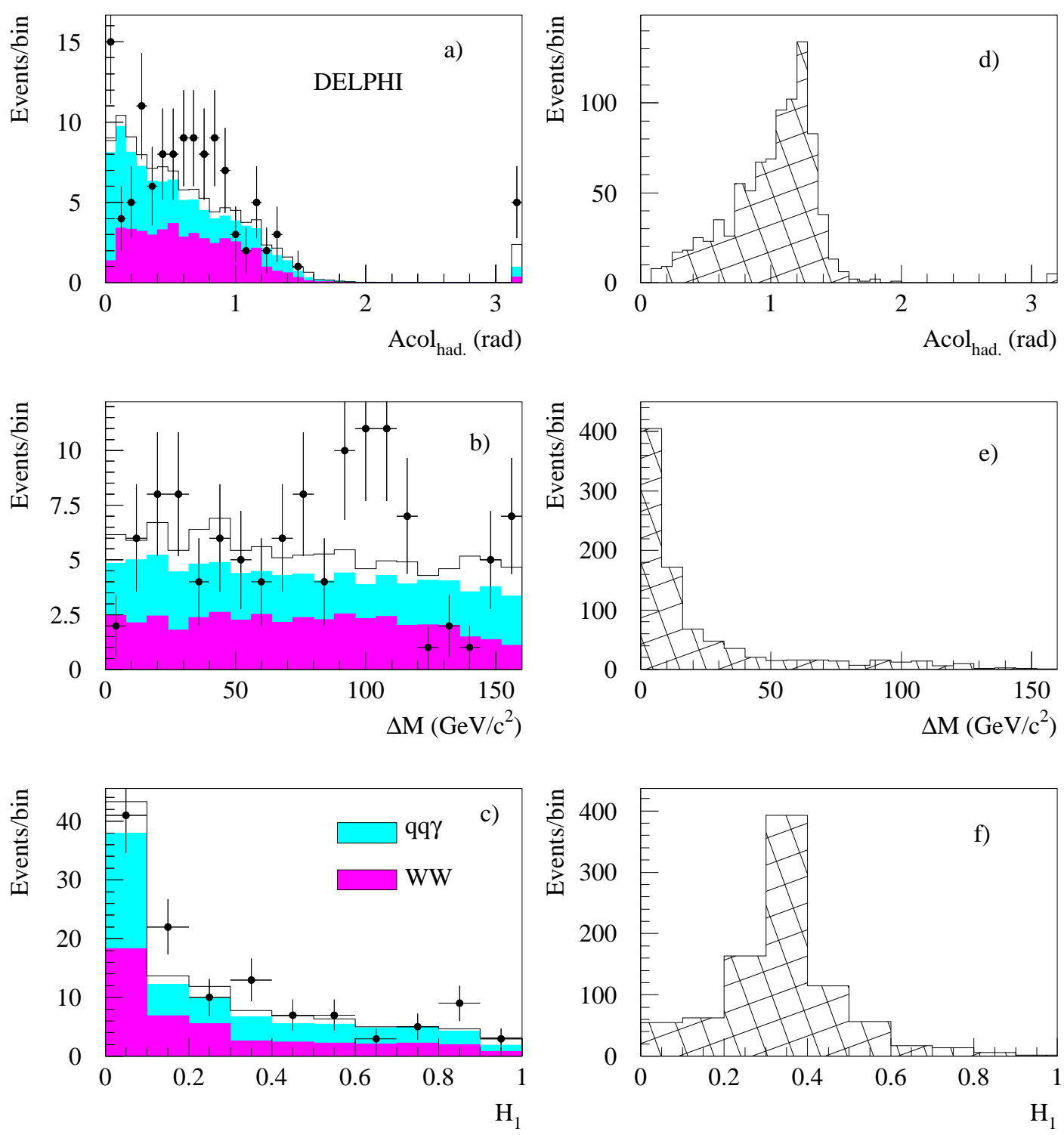

Figure 18: hA $\rightarrow \tau^{+} \tau^{-} \mathrm{q} \overline{\mathrm{q}}$ channel: in plots a) to c), data (dots) are compared to expected background distributions (solid line) at $\sqrt{s}=183 \mathrm{GeV} / c^{2}$. The clear histogram is all other backgrounds. The shapes of the distributions (unnormalized) expected for a Higgs signal at $70 \mathrm{GeV} / c^{2}(\tan \beta=20)$ are shown in $\left.\mathrm{d}\right)$ to $\left.\mathrm{f}\right)$. 


\section{Higgs boson searches in events with purely hadron- ic jets}

The preselection procedure is the same for all four-jet channels. Its aim is to reduce the $q \bar{q}(\gamma)$ background while keeping most of the Higgs boson signal.

The first step is to select hadronic events by requiring at least eighteen charged particles, a total energy above $0.6 \sqrt{s}$ and a total neutral energy below $0.5 \sqrt{s}$. This selection eliminates all Bhabha events and almost all two-photon events without affecting the signal.

To reject events where an on-shell $\mathrm{Z}$ is produced with a real $\gamma$, it is required that no photon with more than $30 \mathrm{GeV}$ is present. This is applied either to photons observed in the calorimeters, or initial state radiation implied by a 3C kinematic fit to the observed jets.

Then four-jet events are selected by demanding three conditions: firstly, the sum of the Fox-Wolfram moments of order two and four has to be less than 1.1; secondly, the thrust of the event has to be less than 0.92. Finally, the DURHAM [20] algorithm is applied, forcing the clusters into a four-jet configuration. These jets are then used in both the following analyses. Each jet is required to contain at least one charged particle and have a mass greater than $1.5 \mathrm{GeV} / c^{2}$. Figure 19 shows the distributions of some of these variables.

\begin{tabular}{|c|c|c|c|c|c|c|}
\hline Selection & Data & Total & & & & $\mathrm{Hq} \overline{\mathrm{q}}$ \\
\hline criteria & & background & $\mathrm{q} \overline{\mathrm{q}}(\gamma)$ & 4-fermion & others & $\varepsilon(\%)$ \\
\hline multi-hadronic & 2303 & 2257 & $1693 \pm 3 \pm 70$ & $558 \pm 3 \pm 23$ & $7 \pm 2$ & $97.2 \pm 0.2$ \\
\hline non-radiative & 1378 & 1371 & $932 \pm 2 \pm 38$ & $438 \pm 2 \pm 18$ & $1.1 \pm 0.5$ & $94.4 \pm 0.3$ \\
\hline four jets & 603 & 617 & $237 \pm 1 \pm 10$ & $380 \pm 2 \pm 16$ & $<1$ & $89.9 \pm 0.4$ \\
\hline
\end{tabular}

Table 18: Hq $\bar{q}$ channel: remaining simulated background event rates and simulated signal efficiencies after each step of the four-jet selection. The first error is statistical and the second is systematic. The signal efficiencies are quoted for a SM Higgs of mass $85 \mathrm{GeV} / c^{2}$.

Table 18 summarises the four-jet preselection performance. The systematic errors have been estimated by taking into account the errors on the cross-sections and the observed differences between generators $(2 \%)$, and the systematic discrepancy found during the selection (slight excess of data at the multi-hadron level and slight loss of data at the four-jet selection level, estimated to be of the order of $3 \%$ ) resulting in a total relative systematic error of $4 \%$. The efficiencies for the generated signal samples range from $81 \%$ to $93 \%$.

\subsection{The Standard Model Higgs selection}

A probabilistic analysis has been used to search for a $\mathrm{ZH}$ signal in the four-jet channel. In order to reduce the main background sources $\left(\mathrm{e}^{+} \mathrm{e}^{-} \rightarrow \mathrm{q} \overline{\mathrm{q}}(\gamma), \mathrm{W}^{+} \mathrm{W}^{-}\right.$and $\left.\mathrm{ZZ}\right)$ topological, kinematical and b-tagging information have been used. 

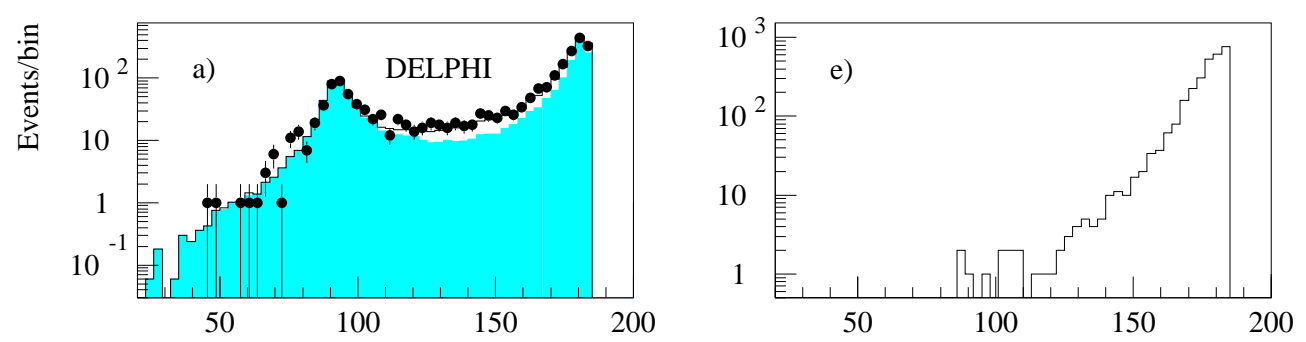

Effective centre-of-mass energy $(\mathrm{GeV})$

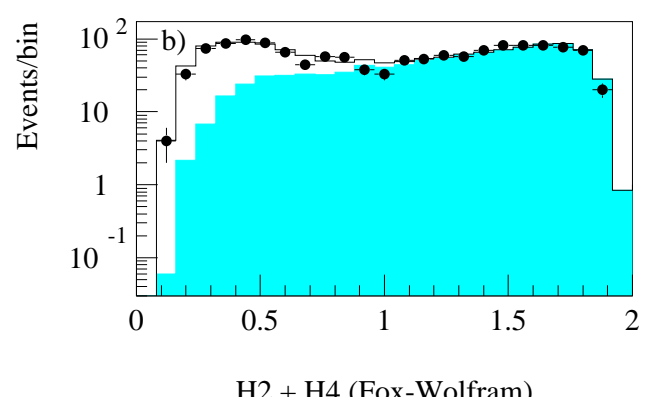

Effective centre-of-mass energy $(\mathrm{GeV})$
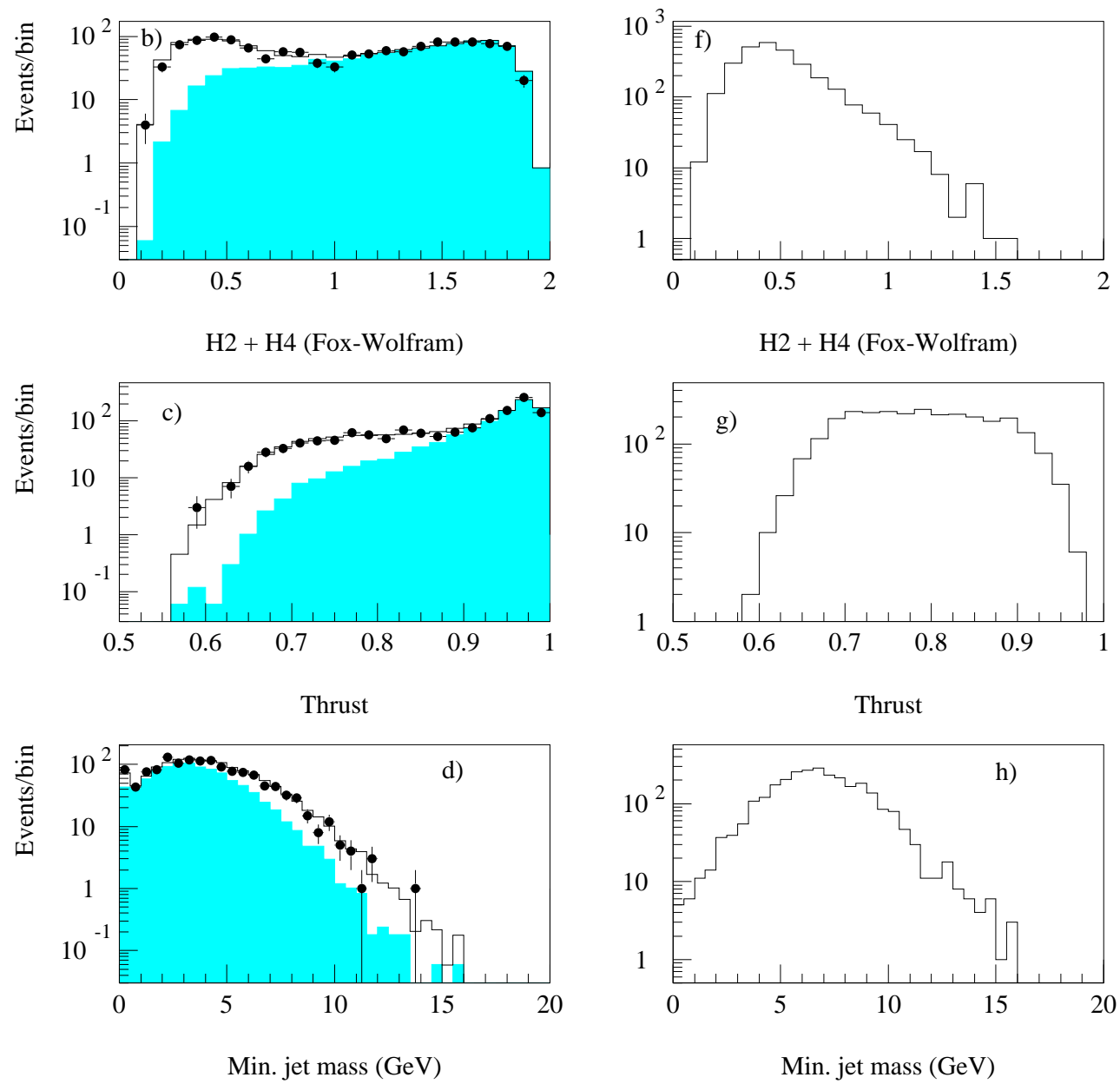

Figure 19: $\mathrm{Hq} \overline{\mathrm{q}}$ channel: distributions of some four-jet preselection variables. Plots a) to d) show comparisons between data (dots) and all simulated background events (solid line) as well as the $q \bar{q}(\gamma)$ background (shaded histogram) normalized to the experimental integrated luminosity. Plots e) to h) show the (unnormalized) expected distributions for the hq $\bar{q}$ process with $m_{\mathrm{H}}=85 \mathrm{GeV} / c^{2}$. a) and b) are made after the initial multi-hadronic selection; the other plots have the non-radiative requirement in addition. 


\subsection{1 b-tagging and kinematical criteria: finding the best jet pairing}

In a four-jet final state there are six combinations of jet pairs which are possible for the Higgs and the $\mathrm{Z}$ bosons. For a signal event, by definition, $i$ and $j$ jet indices will be used for the Higgs bosons whereas $k$ and $l$ indices correspond to the $\mathrm{Z}$ decays.

$\mathcal{P}_{b}\left(x_{\mathrm{b}}^{i}\right)$ is the probability density of the measured value, $x_{\mathrm{b}}^{i}$, for the b-tagging variable for the jet $i$ coming from a $\mathrm{b}$ quark jet. This probability density has been evaluated using simulated samples of $\mathrm{Z}$ decays to $\mathrm{b}$ and non-b quarks. It has been checked that the probabilities for $b$ and non-b quark jets of genuine four jet final states at high energy are essentially identical to these[27].

Effects from the acceptance of the VD have been included by considering three intervals in polar angle inside which the shapes of $\mathcal{P}_{b}\left(x_{\mathrm{b}}^{i}\right)$ have been independently adjusted. These intervals are $\theta \leq 30^{\circ}, 30^{\circ} \leq \theta \leq 40^{\circ}, \theta \geq 40^{\circ}$ and the symmetric intervals obtained by reflection through the origin.

To test the signal hypothesis it has been assumed that the jets $(i, j)$ from the Higgs boson candidate are from $b$ quarks. The two jets from the $\mathrm{Z}$ decays can be initiated by $\mathrm{b}$ or lighter flavours with respective probabilities $\mathcal{P}_{Z}^{b}$ and $\left(1-\mathcal{P}_{Z}^{b}\right)$, given by the $\mathrm{Z}$ couplings to the different hadronic final states. Finally the $\chi^{2}$ probability of the $5 \mathrm{C}$ fit, obtained by imposing that the mass of the di-jet $(k, l)$ be equal to the $\mathrm{Z}$ mass, is included to define the probability that the four jets present in the event correspond to the channel $e^{+} e^{-} \rightarrow$ $\mathrm{ZH}$ :

$$
\begin{aligned}
\mathcal{P}_{Z H}^{b}\left(x_{b}^{1}, x_{b}^{2}, x_{b}^{3}, x_{b}^{4}\right)=\sum \mathcal{P}_{b}\left(x_{b}^{i}\right) \mathcal{P}_{b}\left(x_{b}^{j}\right)\left[\left(1-\mathcal{P}_{Z}^{b}\right) \mathcal{P}_{q}\left(x_{b}^{k}\right) \mathcal{P}_{q}\left(x_{b}^{l}\right)\right. & \left.+\mathcal{P}_{Z}^{b} \mathcal{P}_{b}\left(x_{b}^{k}\right) \mathcal{P}_{b}\left(x_{b}^{l}\right)\right] \\
& \times \mathcal{P}_{\chi^{2}}^{5 C}\left(m_{k l}=m_{Z}\right)
\end{aligned}
$$

The sum runs over the six different possible pairings of the jets $i, j, k, l$. In this expression $\mathcal{P}_{Z}^{b}=0.216, \mathcal{P}_{q}\left(x_{b}^{l}\right)=1-\mathcal{P}_{b}\left(x_{b}^{l}\right)$ and the sum, over the six combinations, of the $5 \mathrm{C}$ fit $\chi^{2}$ probabilities has been normalized to unity.

In a similar way, the probability that the observed event comes from the $q \bar{q}(\gamma)$ background has been evaluated:

$$
\begin{aligned}
\mathcal{P}_{\mathrm{q} \overline{\mathrm{q}}(\gamma)}^{b}\left(x_{b}^{1}, x_{b}^{2}, x_{b}^{3}, x_{b}^{4}\right)=\frac{1}{6} \sum \quad & \left(1-\mathcal{P}_{\gamma}^{b}\right) \mathcal{P}_{q}\left(x_{b}^{i}\right) \mathcal{P}_{q}\left(x_{b}^{j}\right) \mathcal{P}_{q}\left(x_{b}^{k}\right) \mathcal{P}_{q}\left(x_{b}^{l}\right) \\
& \left.+\mathcal{P}_{\gamma}^{b} \mathcal{P}_{b}\left(x_{b}^{i}\right) \mathcal{P}_{b}\left(x_{b}^{j}\right) \mathcal{P}_{q}\left(x_{b}^{k}\right) \mathcal{P}_{q}\left(x_{b}^{l}\right)\right]
\end{aligned}
$$

In this expression the fraction of b $\bar{b}$ jets in a di-jet, $\mathcal{P}_{\gamma}^{b}$, the fraction of jets in $q \bar{q}(\gamma)$ events which are from $b$ quarks, has been taken from the simulation $\left(\mathcal{P}_{\gamma}^{b}=0.175\right)$. The $(i, j)$ and $(k, l)$ masses are not constrained.

For $\mathrm{W}^{+} \mathrm{W}^{-}$events, neglecting the very small $\mathrm{W}^{-} \rightarrow \mathrm{b} \overline{\mathrm{c}}(\overline{\mathrm{u}})$ couplings and the difference between the fractions of $\mathrm{c}$ quarks in $\mathrm{W}$ and in $\mathrm{Z}$ decays which was assumed to define $\mathcal{P}_{b}\left(x_{b}^{i}\right)$, the following expression is obtained:

$$
\mathcal{P}_{W W}^{b}\left(x_{b}^{1}, x_{b}^{2}, x_{b}^{3}, x_{b}^{4}\right)=\mathcal{P}_{q}\left(x_{b}^{1}\right) \mathcal{P}_{q}\left(x_{b}^{2}\right) \mathcal{P}_{q}\left(x_{b}^{3}\right) \mathcal{P}_{q}\left(x_{b}^{4}\right)
$$

To verify the validity of the approximations concerning the description of $W$ decays, the b-tag probability distributions have also been determined separately for light, charm and $b$ flavours and the different branching fractions of $W$ bosons into $q_{1} \bar{q}_{2}$ pairs have been used. The $\chi^{2}$ probability of a $5 \mathrm{C}$ fit, in which the equality of $(i, j)$ and $(k, l)$ di-jet masses is required, has also been tried in the expression for $\mathcal{P}_{W W}^{b}$. As no significant improvement in the background rejection was found, the simpler expression above was adopted.

No separate $\mathcal{P}_{Z Z}$ probability has been calculated.

The jet pairing which maximises the term of the expression of $\mathcal{P}_{Z H}^{b}$ is selected and the Higgs boson candidate mass is given by the $5 \mathrm{C}$ fit. 


\subsubsection{Contributions from the shape variables}

The previous expressions for $\mathcal{P}_{Z H}^{b}, \mathcal{P}_{\mathrm{q} \overline{\mathrm{q}}(\gamma)}^{b}$ and $\mathcal{P}_{W W}^{b}$ have been multiplied by the probability densities observed for shape variables in the signal and in the two background components. Five variables have been used:

- the minimum di-jet mass;

- $\alpha_{\min } E_{\min }$, which is the product of the minimum jet energy and the minimum opening angle between two jets,

- $\left(H_{2}+H_{4}\right) / H_{0}$, the normalized sum of two Fox-Wolfram moments;

- $\beta_{\min } \cdot E_{\max } / E_{\min }$ where $\beta_{\min }$ is the minimum angle between the most energetic jet, of energy $E_{\max }$, and the others;

- the value of the cut in the jet algorithm, $y_{34}$, corresponding to the transition between three- and four-jet topologies for the studied event.

The distributions of these variables are shown in Fig. 20 at the level of the preselection. A good agreement between data and simulation in the variables used is observed. Distant tails in these distributions have been replaced by a constant level in the parametrization functions. All the signal simulations for different values of the Higgs boson mass between 70 and $95 \mathrm{GeV} / c^{2}$ have been used to produce the signal shapes. This ensures a rather constant acceptance of the signal inside this mass range.

\subsubsection{Final event selection}

A discriminating variable has been defined as the ratio of two likelihood distributions obtained, respectively, for signal and background simulated events. The discriminating variable used in this analysis is then:

$$
X_{\mathrm{disc}}=\frac{\mathcal{P}_{Z H}}{\mathcal{P}_{W} \mathcal{P}_{W W}+\left(1-\mathcal{P}_{W}\right) \mathcal{P}_{\mathrm{q} \overline{\mathrm{q}}(\gamma)}}
$$

$\mathcal{P}_{W}$ is the fraction of $\mathrm{W}^{+} \mathrm{W}^{-}$pairs in the preselected sample $\left(\mathcal{P}_{W}=0.575\right)$ deduced from the simulation. The quantities $\mathcal{P}_{Z H}, \mathcal{P}_{W W}$ and $\mathcal{P}_{\mathrm{q} \overline{\mathrm{q}}(\gamma)}$ are the products of the expressions $\mathcal{P}^{b}\left(x_{1}^{b}, x_{2}^{b}, x_{3}^{b}, x_{4}^{b}\right)$ defined previously by the probability distributions for the shape variables obtained for the three classes of events. The background component coming from production of two neutral bosons ( $Z$ 's or $\gamma$ 's) has not been considered in the expression of the background probability distribution because it behaves like $q \bar{q}(\gamma)$ events $\left(\mathrm{Z} \gamma^{*}\right.$ component) or like signal events (on-shell ZZ pair events when a $\mathrm{Z}$ decays into $\mathrm{b} \overline{\mathrm{b}})$. The distributions of the decimal logarithm of the discriminating variable obtained in data and in simulated events are compared in Fig. 21.

\subsubsection{Comparison between data and simulation}

The final event selection is made through a cut on the discriminating variable. The efficiency and background as a function of the cut can be seen in Fig. 22. The efficiencies at different levels of this selection, for a Higgs boson of $85 \mathrm{GeV} / c^{2}$ mass, and the number of kept events from the different background components are summarized in Table 19, for three different values of the cut.

The value of the final cut on this variable is fixed by the global optimization algorithm. A requirement $X_{\text {disc }}>100$ is found to correspond to the optimal working point, selecting one event in the data while $3.74 \pm 0.20 \pm 0.18$ are expected from standard processes. The values of the corresponding efficiencies to the signal, for different values of the Higgs 

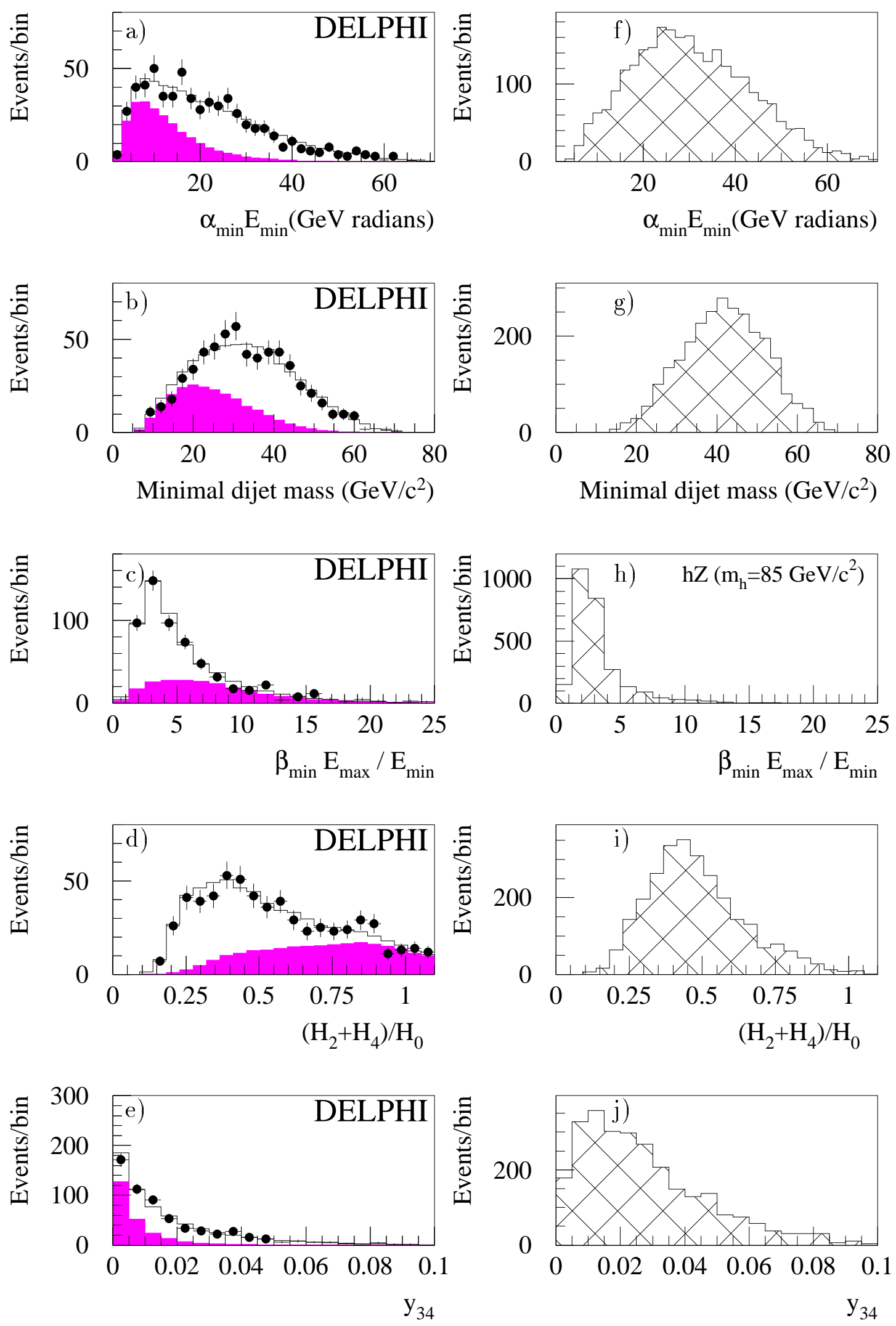

Figure 20: $\mathrm{Hq}_{\bar{q}}$ channel: a) to e) are the topological variable distributions for the data and the expected background and f) to j) are the same for a $\mathrm{ZH}$ signal with $m_{\mathrm{H}}=$ $85 \mathrm{GeV} / c^{2}$. Dots show the data and the line is the total background. Shaded histograms correspond to the $q \bar{q}(\gamma)$ background component. 


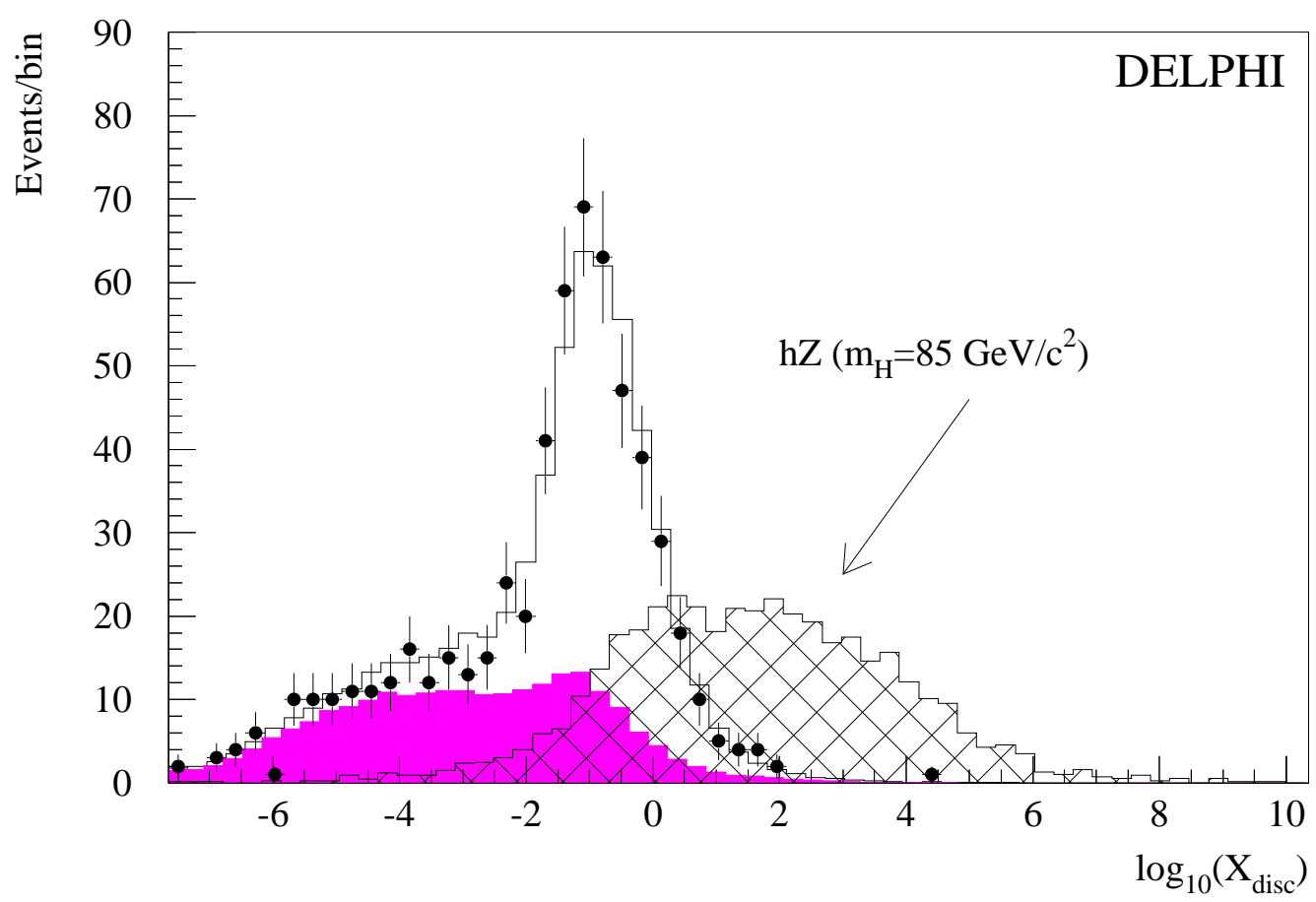

Figure 21: Hqव channel: distribution of the decimal logarithm of the discriminating variable $X_{\text {disc }}$ obtained in data (dots) recorded around $\sqrt{\mathrm{s}}=183 \mathrm{GeV}$ and in simulated events (solid line is total, $\mathrm{q} \overline{\mathrm{q}}(\gamma)$ component shaded). The hatched distribution corresponds, with arbitrary normalization, to the channel $\mathrm{e}^{+} \mathrm{e}^{-} \rightarrow \mathrm{ZH}$, with $m_{\mathrm{H}}=85 \mathrm{GeV} / c^{2}$.

\begin{tabular}{lccccccc}
\hline & Data & $\begin{array}{c}\text { All } \\
\text { background }\end{array}$ & $\mathrm{q} \overline{\mathrm{q}}(\gamma)$ & WW & ZZ & $\begin{array}{c}\text { Zee and } \\
\text { others }\end{array}$ & $\begin{array}{c}\text { ZH } \\
\text { Efficiency (\%) }\end{array}$ \\
\hline $\log _{10}\left(X_{\text {disc }}\right) \geq-1.4$ & 336 & $329.6 \pm 2.3 \pm 16.6$ & 60.5 & 258.3 & 10.9 & 1.4 & $84.6 \pm 0.7$ \\
\hline $\log _{10}\left(X_{\text {disc }}\right) \geq-0.75$ & 169 & $176.6 \pm 1.7 \pm 14.5$ & 29.7 & 139.3 & 7.5 & 0.2 & $78.4 \pm 0.8$ \\
\hline $\log _{10}\left(X_{\text {disc }}\right) \geq 2$ & 1 & $3.74 \pm 0.20 \pm 0.18$ & 1.64 & 0.80 & 1.30 & 0 & $38.1 \pm 0.9$ \\
\hline
\end{tabular}

Table 19: Hq $\bar{q}$ channel: number of selected multi-hadronic events in data and corresponding expectations from the simulation at different levels of the analysis. The last column gives the selection efficiency for the channel $\mathrm{e}^{+} \mathrm{e}^{-} \rightarrow \mathrm{ZH} \rightarrow$ hadrons, using $m_{\mathrm{H}}=85 \mathrm{GeV} / c^{2}$.

\begin{tabular}{cccc}
\hline$m_{\mathrm{H}}\left(\mathrm{GeV} / c^{2}\right)$ & Efficiency $(\%)$ & $m_{\mathrm{H}}\left(\mathrm{GeV} / c^{2}\right)$ & Efficiency $(\%)$ \\
\hline 60 & $28.9 \pm 1.0 \pm 0.4$ & 80 & $35.5 \pm 0.9 \pm 0.5$ \\
65 & $29.6 \pm 1.0 \pm 0.4$ & 85 & $38.1 \pm 0.9 \pm 0.5$ \\
70 & $31.2 \pm 0.8 \pm 0.4$ & 90 & $40.5 \pm 0.9 \pm 0.5$ \\
75 & $35.7 \pm 1.1 \pm 0.5$ & 95 & $36.4 \pm 1.1 \pm 0.5$ \\
\hline
\end{tabular}

Table 20: Hq $\bar{q}$ channel: efficiency of the Higgs boson selection as a function of the mass of the Higgs boson. 


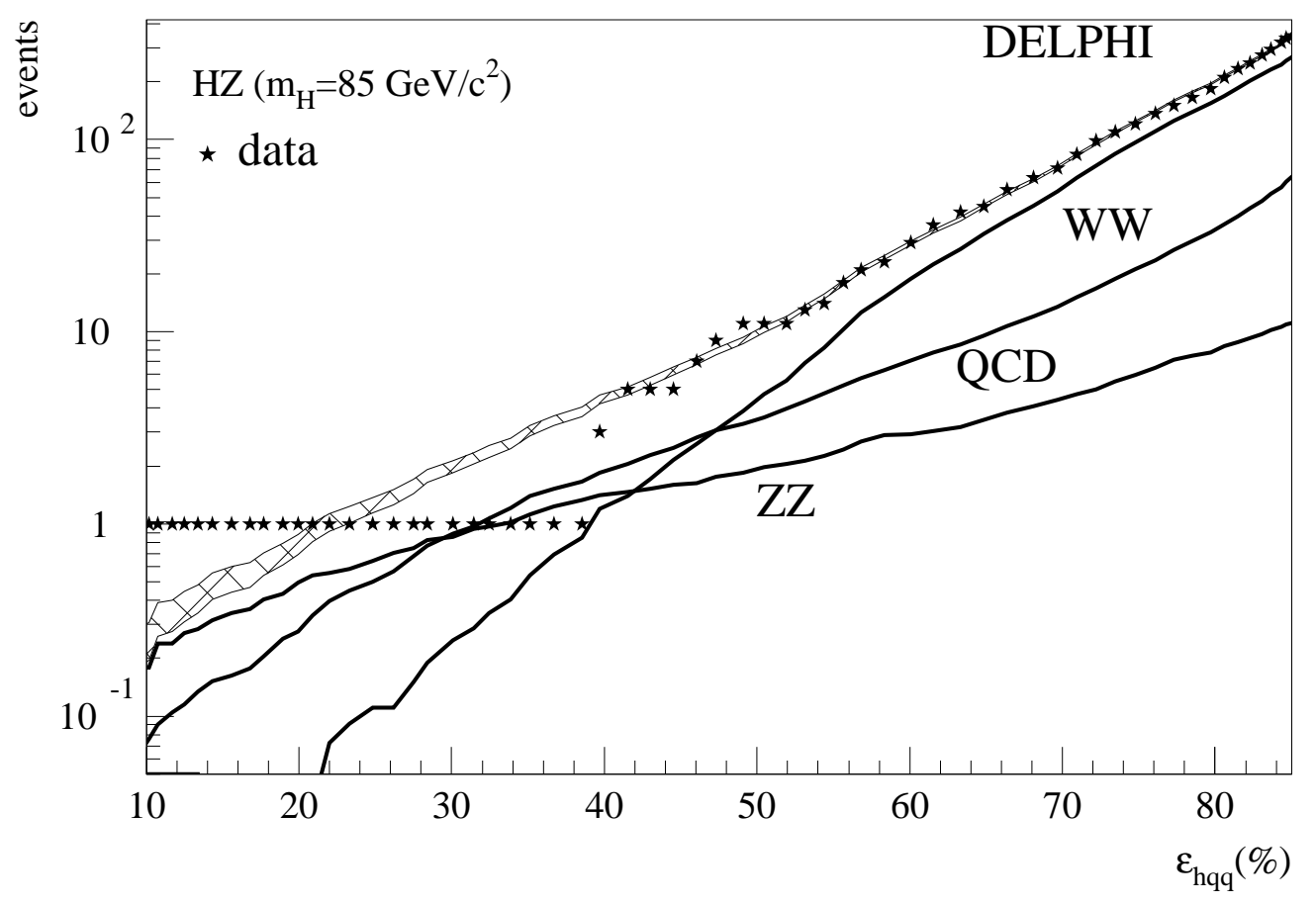

Figure 22: $\mathrm{Hq} \overline{\mathrm{q}}$ channel: variation of the expected number of background events versus the efficiency for a Higgs signal of $85 \mathrm{GeV} / c^{2}$ mass, at $\sqrt{s}=183 \mathrm{GeV}$, obtained by varying the cut on the $\log _{10}\left(X_{\text {disc }}\right)$ variable. The expected numbers of $q \bar{q}(\gamma), W W$ and ZZ background events are shown separately. Statistical errors on the expected number of background events are indicated by the hatched band corresponding to the sum of all background components. 
boson mass can be found in Table 20 and Fig. 29. The mass distributions of the selected Higgs di-jet candidates, obtained after the 5-C fit, in data and in the simulation have been compared at different levels of the selection in Fig. 23. The data event passing through the selection has a measured Higgs mass of $89.4 \mathrm{GeV} / c^{2}$ and a $\log _{10}\left(X_{\text {disc }}\right)$ of 4.4. It can be seen in Fig. 24.
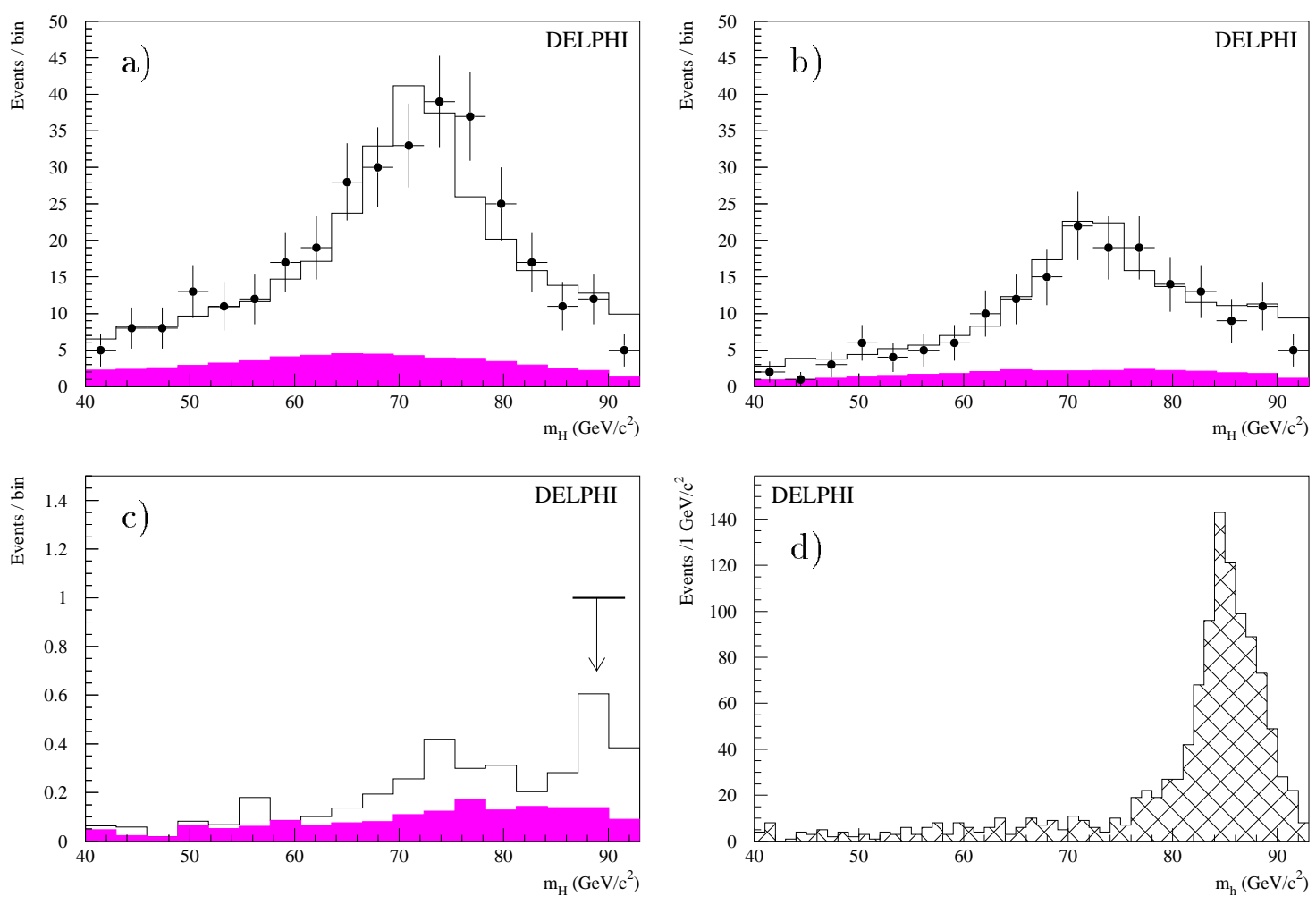

Figure 23: $\mathrm{Hq} \overline{\mathrm{q}}$ channel: mass spectra measured in data and in the expected background for the Higgs di-jet candidate at different levels of the selection: a) efficiency $\sim 85 \%, b$ ) efficiency $\sim 79 \%$ and c) optimal working point. Dots are the data, and the solid line is the total background. Shaded histograms correspond to the $q \bar{q}(\gamma)$ background component, and the arrow in c) shows the position of the candidate. The lower right histogram $\mathrm{d}$ ) corresponds to the expected mass spectrum for a $85 \mathrm{GeV} / c^{2}$ Higgs boson, at the working point.

\subsubsection{Systematic uncertainties}

Systematic uncertainties on the background evaluation may originate from differences between data and simulation in the rates, event shape or b-tagging distributions for the different components. All studied distributions in data and simulation have been found to be in agreement within statistics. A $\pm 4 \%$ systematic uncertainty has been evaluated on the preselection requirements (Sect. 8). The discriminating variable (shown in Fig. 21) has a different behaviour for $q \bar{q}(\gamma), \mathrm{W}^{+} \mathrm{W}^{-}$and $\mathrm{ZZ}$ events and the relative fractions of the three components have been fitted to data after the preselection and found to be in agreement with expectations (Table 21). This fit assumed no Higgs boson production, but much of the information comes from regions of low $\log _{10}\left(X_{\mathrm{disc}}\right)$, which are not affected by a possible signal. 


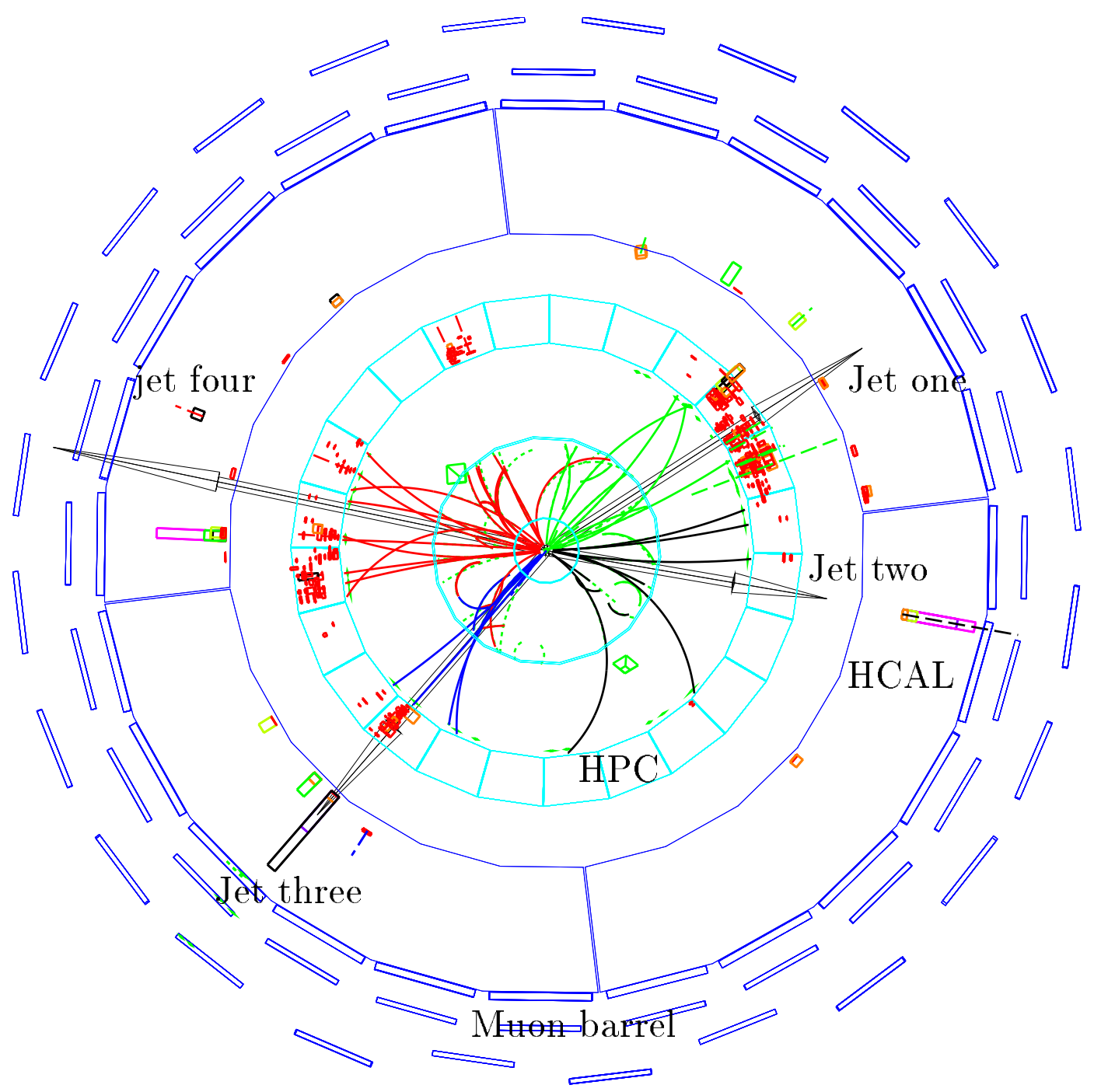

Figure 24: $\mathrm{Hq} \overline{\mathrm{q}}$ channel: transverse view of the surviving candidate. The arrows show the jet directions and momenta, and the tracks in each of the four jets are shaded differently. 


\begin{tabular}{lccc}
\hline & $N_{W W}$ & $N_{\mathrm{q} \overline{\mathrm{q}}(\gamma)}$ & $N_{Z Z}$ \\
\hline Measured & $366_{-22}^{+21}$ & $227_{-21}^{+22}$ & $6_{-6}^{+13}$ \\
\hline Expected & 364.6 & 234.6 & 19.1 \\
\hline
\end{tabular}

Table 21: Fitted and expected numbers of events corresponding to the different background components.

From this result, which is also sensitive to possible differences in event shapes between the data and the simulation, relative uncertainties of $\pm 6 \%$ and $\pm 10 \%$ have been estimated respectively on the relative rates of $\mathrm{W}^{+} \mathrm{W}^{-}$and $\mathrm{q} \overline{\mathrm{q}}(\gamma)$ backgrounds. No systematic uncertainty needs to be added for the $\mathrm{ZZ}$ component because the uncertainty on its importance is, at present, naturally dominated by statistics. The present evaluation quoted in Table 21 indicates that there is no large discrepancy as compared to expectations for this component. The distributions of the b-tag variable measured in data and in the simulation have been compared for preselected events (see Fig. 25). A difference is observed in the region dominated by light flavours. To gain in statistics, in the region mainly populated by b quarks, similar distributions have been studied using Z decays. They confirm the effect observed at high energy in the light flavour populated region, and do not show evidence for a discrepancy in the b-quark region. Simulated events have been modified to correct for the observed difference. The measured relative variations on the efficiency of the signal and the expected background are respectively $\pm 1.3 \%$ and $\pm 2.2 \%$, and these are included in the quoted systematics.

\subsection{The hA four-b channel}

A neural network has been used to search for hA production in the four-jet channel. The common four-jet selection criteria are applied to preselect relevant events with the exception of the requirement on $H_{2}+H_{4}$. Instead, this quantity is used as input for the neural network. The resulting number of expected events and the signal efficiencies after the preselection are given in Table 22 .

\begin{tabular}{cccccc}
\hline \multicolumn{3}{c}{ Total } & & \multicolumn{2}{c}{ hA Efficiency $(\%)$} \\
Data & background & $\mathrm{q} \overline{\mathrm{q}}(\gamma)$ & WW & ZZ & $m_{\mathrm{A}}=70 \mathrm{GeV} / c^{2}, \tan \beta=20$ \\
\hline 655 & $686 \pm 27$ & 311 & 355 & 20 & $93 \%$ \\
\hline
\end{tabular}

Table 22: hA 4b channel: the number of expected background events after the preselection. The number of events found in data is also given.

\subsubsection{Input variables for the neural network}

The input variables were selected in a pre-analysis using a discriminant analysis technique. The following 14 variables are used:

- $\ln (6+$ Event combined b-tag for all jets $)$,

- $\ln (5+$ Event combined b-tag for the two jets with highest b-tag), 


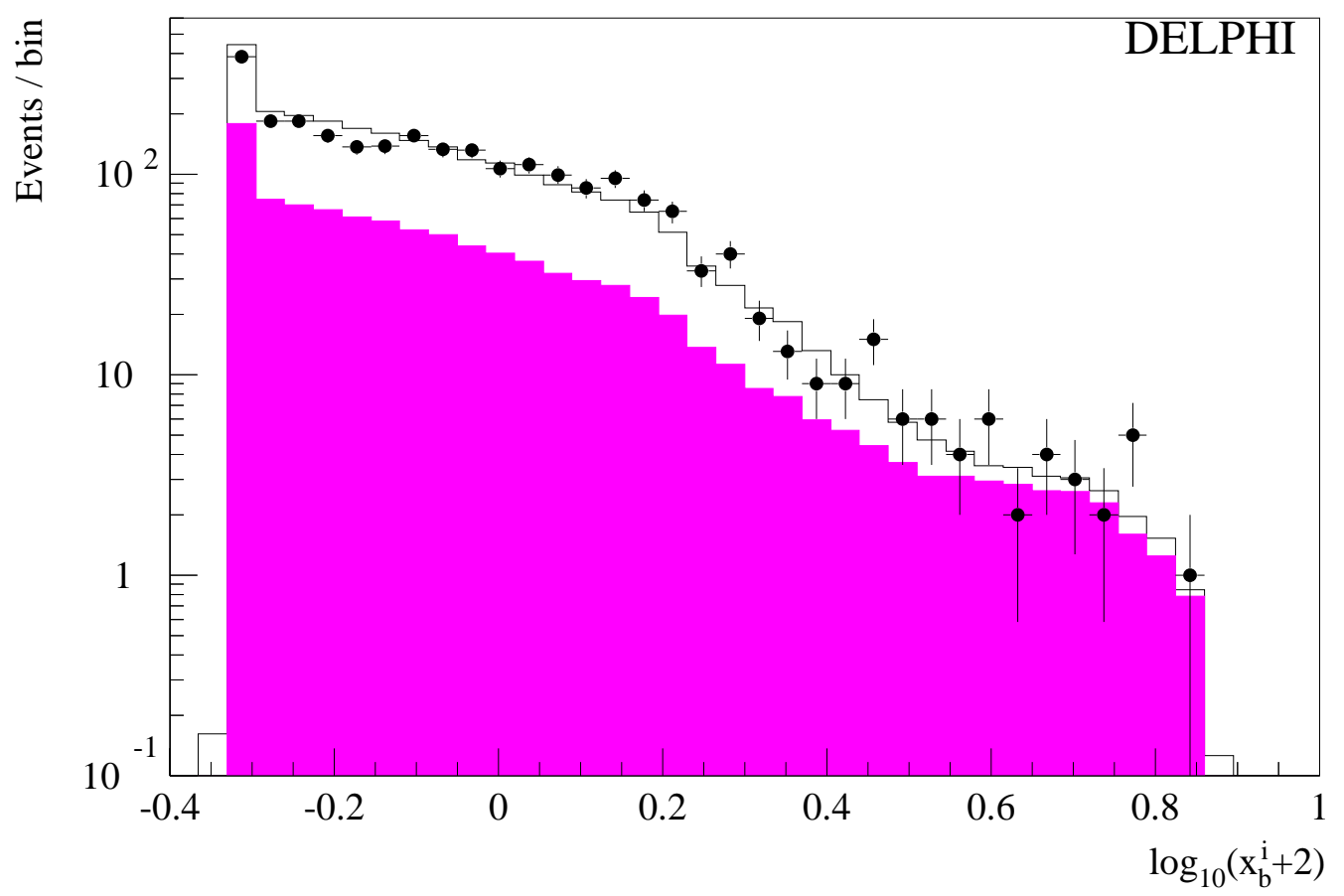

Figure 25: Hq $\bar{q}$ channel: distributions of the decimal logarithm of the b-tagging variable defined for each jet at the preselection level. Lower values correspond to light quark flavours while higher values correspond mainly to b quarks. The data is shown with dots and the simulated background with the upper line. The shaded histogram corresponds to the $q \bar{q}(\gamma)$ background component. 
- combined jet b-tag of the jet with highest b-tag probability,

- combined jet b-tag of the jet with third highest b-tag probability,

- number of secondary vertices,

- thrust,

- $2-\mathrm{H}_{2}-\mathrm{H}_{4}$,

- minimal jet charged multiplicity,

- $-\log _{10}\left(y_{34}\right)$ (DURHAM),

- minimal di-jet mass

- $\beta_{\text {min }}$, defined as the minimal angle between the most energetic jet and the three remaining jets,

- maximum jet energy,

- minimum jet energy,

- minimum angle between any two jets.

Event b-tag variables are transformed as given above in order to have less peaked distributions. All inputs are normalized to lie in the region between zero and one. Then they are fed into a fully connected three-layer feed-forward percepton with 14 nodes in the input, 15 nodes in the hidden and 1 node in the output layer, using the JETNET package [28]. The training sample has been subdivided into two subsamples, one of them is used for the training, and the second one to monitor the performance of the network. The two samples included $3000 \mathrm{q} \overline{\mathrm{q}}(\gamma), 3000 \mathrm{WW}$ and $1000 \mathrm{ZZ}$ events for the background, and 900 events for each of the Higgs masses from 60 to $85 \mathrm{GeV} / c^{2}(\tan \beta=20)$.

Figure 26 a) shows the network output for a natural mixture of the three background types $\mathrm{q} \overline{\mathrm{q}}(\gamma)$, WW and ZZ. The output distribution for a signal with $m_{\mathrm{A}}=70 \mathrm{GeV} / c^{2}$ and $\tan \beta=20$ is also given.
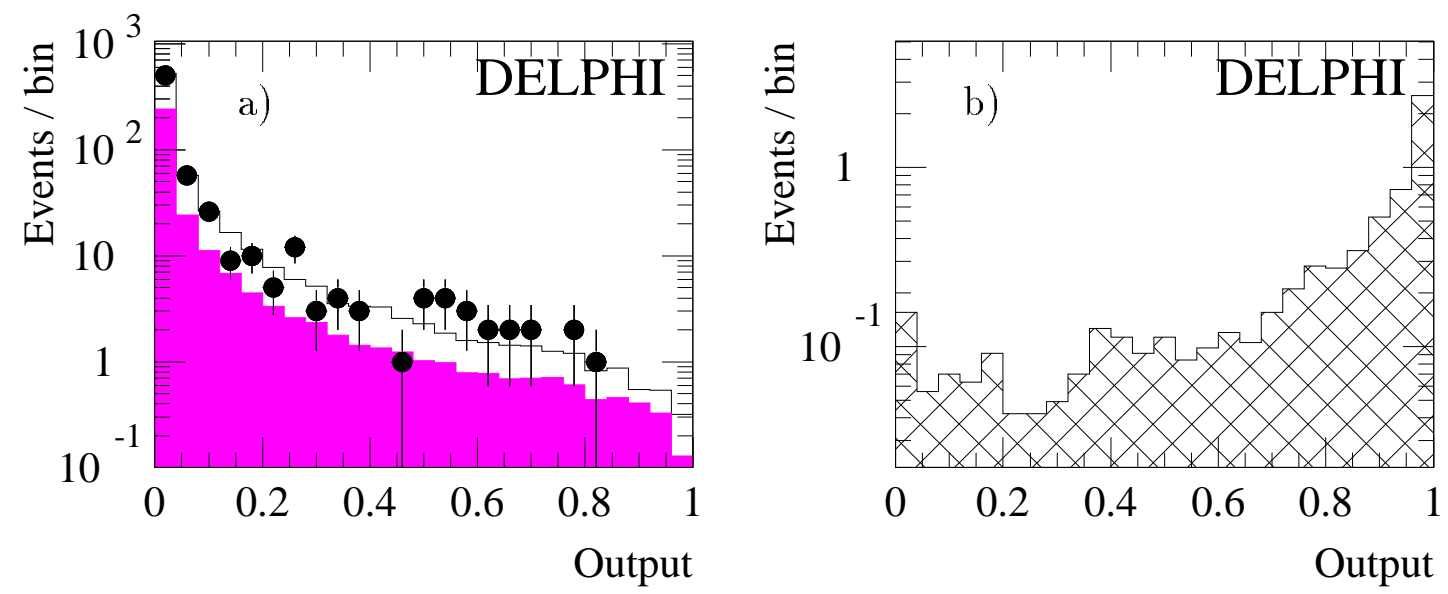

Figure 26: hA $\rightarrow \mathrm{b} \bar{b} b \bar{b}$ channel: the output of the neural network a) for background (solid line) and data (dots). The $q \bar{q}(\gamma)$ background contribution is shaded. Plot b) shows the expectation for a $m_{\mathrm{A}}=70 \mathrm{GeV} / c^{2}$ and $\tan \beta=20$.

\subsubsection{Final optimization}

The final selection on the neural network output is chosen by the global optimization procedure (see Sect. 4.5) and corresponds to a requirement at 0.877 . This yields a total resulting background of $1.46 \pm 0.13$ events, coming from $q \bar{q}(\gamma)(0.906 \pm 0.071)$, WW $(0.155 \pm 0.058)$ and $\mathrm{ZZ}(0.403 \pm 0.097)$. No events are selected in the data. Efficiencies 

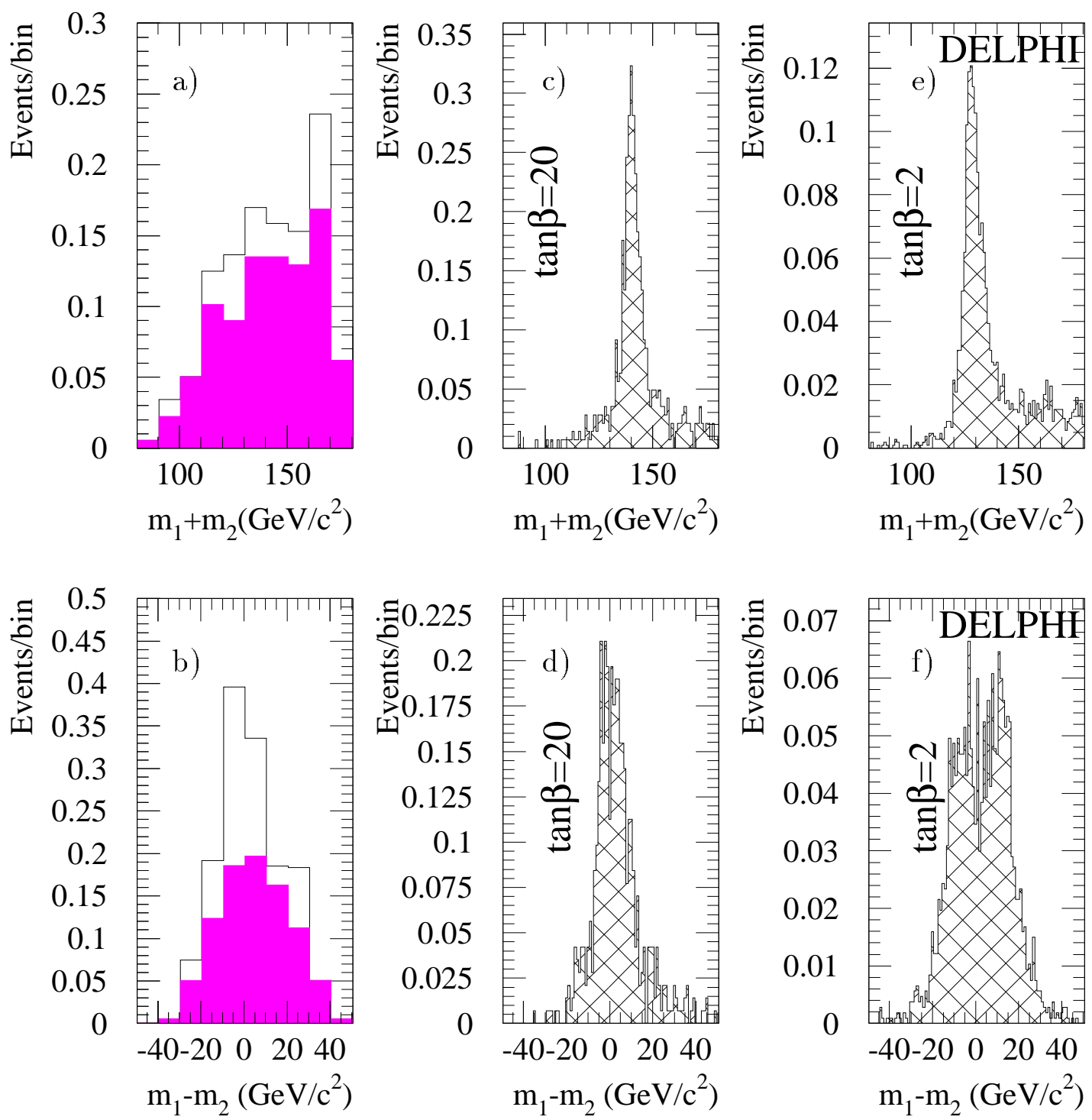

Figure 27: hA $\rightarrow$ b $\bar{b} b \bar{b}$ channel: $\operatorname{sum}(a), c)$ and e)) and difference (b), d) and f)) of the di-jet masses after all the cuts of the hA analysis with neural networks. The expected backgrounds, in events per bin, are in a) and b), with the total background given by the solid line and the $q \bar{q}(\gamma)$ component shaded. The signal shape, with arbitrary normalisation and $m_{\mathrm{A}}=70 \mathrm{GeV} / c^{2}$ is shown in c) and d) for $\tan \beta=20$ and e) and $\mathrm{f}$ ) for $\tan \beta=2$. In the first case the mass of the $\mathrm{h}$ is $69.7 \mathrm{GeV}$, while in the second it is $60.8 \mathrm{GeV}$ and the larger difference explains the larger width in $\mathrm{f}$ ). The distributions are given for the jet pairings with the smallest mass difference. 


\begin{tabular}{cll}
\hline Mass & \multicolumn{2}{c}{ Efficiency $(\%)$} \\
$m_{\mathrm{A}}\left(\mathrm{GeV} / c^{2}\right)$ & $\tan \beta=20$ & $\tan \beta=2$ \\
\hline 55 & $43.1 \pm 1.1 \pm 1.2$ & $40.2 \pm 1.1 \pm 1.2$ \\
60 & $48.3 \pm 1.6 \pm 0.9$ & $45.3 \pm 1.1 \pm 1.2$ \\
65 & $52.6 \pm 1.6 \pm 1.2$ & $49.0 \pm 1.1 \pm 0.9$ \\
70 & $55.0 \pm 1.6 \pm 0.9$ & $50.5 \pm 0.8 \pm 1.3$ \\
75 & $55.8 \pm 1.1 \pm 1.4$ & $53.4 \pm 0.9 \pm 1.2$ \\
80 & $54.3 \pm 1.1 \pm 1.3$ & $52.8 \pm 0.9 \pm 1.3$ \\
85 & $53.4 \pm 1.6 \pm 1.4$ & $55.4 \pm 0.8 \pm 1.3$ \\
\hline
\end{tabular}

Table 23: hA 4b channel: efficiency for different masses of the pseudo-scalar Higgs. Errors are statistical and systematic.

obtained for all masses and $\tan \beta$ are summarized in Table 23. Figure 27 shows the distributions of the sum and the difference of the di-jet masses at the end of the analysis for $m_{\mathrm{A}}=70 \mathrm{GeV} / c^{2}$ and $\tan \beta=2$ or 20 . In these distributions, the jet pairing is that with the smallest di-jet mass difference. Figure 27 shows that this pairing allows for a good reconstruction of the sum of the di-jet masses even when the $\mathrm{h}$ and $\mathrm{A}$ bosons have a (moderate) mass difference.

\subsubsection{Systematic errors}

The distributions of the input variables shows no significant difference between data and simulation after preselection. A small discrepancy is observed in the highest jet b-tag probability distribution in the light quark region. An additional preselection cut which removes this region does not affect signal and background selection efficiencies. Each event of the testing sub-sample (Sect. 8.2.1) has been shifted by the difference of the mean values between simulation and data. Then the shifted events have been moved randomly fifty times within the error of the mean value of the data distribution. Correlations of the input variables are taken from Monte Carlo simulation. The mean shift of the resulting efficiencies is taken as systematic uncertainty.

To check systematic uncertainties on the total background due to the training procedure the training and the validation sample have been exchanged and the training was repeated. The uncertainty on the total background due to this is of the order $4 \%$. An additional uncertainty on the input variables of $4 \%$ at preselection level has been taken from Sect. 8. Summing all errors in quadrature yields a relative error of $12 \%$ on the total background.

\section{Global optimization and results}

As there is no evidence for a signal, the results of the searches presented in the previous sections translate into exclusion limits on the masses of the neutral Higgs bosons in the SM and MSSM. The procedure used to set the limit is the same as that first used to optimize the expected limit. The results of the global optimization are reported first. 